\title{
MRI of therapeutic neovascularization: morphology \& function
}

Citation for published version (APA):

Jaspers, K. (2011). MRI of therapeutic neovascularization: morphology \& function. [Doctoral Thesis, Maastricht University]. Datawyse / Universitaire Pers Maastricht. https://doi.org/10.26481/dis.20110909kj

Document status and date:

Published: 01/01/2011

DOI:

10.26481/dis.20110909kj

Document Version:

Publisher's PDF, also known as Version of record

\section{Please check the document version of this publication:}

- A submitted manuscript is the version of the article upon submission and before peer-review. There can be important differences between the submitted version and the official published version of record.

People interested in the research are advised to contact the author for the final version of the publication, or visit the DOI to the publisher's website.

- The final author version and the galley proof are versions of the publication after peer review.

- The final published version features the final layout of the paper including the volume, issue and page numbers.

Link to publication

\footnotetext{
General rights rights.

- You may freely distribute the URL identifying the publication in the public portal. please follow below link for the End User Agreement:

www.umlib.nl/taverne-license

Take down policy

If you believe that this document breaches copyright please contact us at:

repository@maastrichtuniversity.nl

providing details and we will investigate your claim.
}

Copyright and moral rights for the publications made accessible in the public portal are retained by the authors and/or other copyright owners and it is a condition of accessing publications that users recognise and abide by the legal requirements associated with these

- Users may download and print one copy of any publication from the public portal for the purpose of private study or research.

- You may not further distribute the material or use it for any profit-making activity or commercial gain

If the publication is distributed under the terms of Article $25 \mathrm{fa}$ of the Dutch Copyright Act, indicated by the "Taverne" license above, 
MRI of therapeutic neovascularization: morphology \& function 
(C) Copyright Karolien Jaspers, Groningen 2011

Printed by Datawyse / Universitaire Pers Maastricht. ISBN: 9789461590725

Cover design: Karolien Jaspers

The waters of Maastricht: The rivers Maas and Jeker and the Albert Canal 


\title{
MRI of therapeutic neovascularization: morphology \& function
}

\author{
PROEFSCHRIFT \\ ter verkrijging van de graad van doctor \\ aan de Universiteit Maastricht, \\ op gezag van de Rector Magnificus, \\ Prof. Mr. G.P.M.F. Mols, \\ volgens het besluit van het College van Decanen \\ in het openbaar te verdedigen op \\ vrijdag 9 september 2011 om 14:00 uur \\ door

\section{Karolien Jaspers} \\ geboren op 27 januari 1981 te Hengelo (O).
}

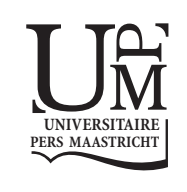


Promotor

Prof. dr. M.J. Post

Copromotores

Dr. ir. W.H. Backes

Dr. T. Leiner

Beoordelingscommissie

Prof. dr. J.M.A. van Engelshoven (voorzitter)

Prof. dr. K. Nicolay (Technische Universiteit Eindhoven)

Prof. dr. G.W.H. Schurink

Prof. dr. H. Vink

Prof. dr. ir. F.N. van de Vosse (Technische Universiteit Eindhoven)

The research described in this thesis was supported by a grant of the Dutch Heart Foundation (DHF-2005B178)

Financial support by the Dutch Heart Foundation for the publication of this thesis is gratefully acknowledged.

Gadomer was kindly supplied by Bayer Schering Pharma AG. 
The mystery of magnetism, explain that to me! No greater mystery, except love and hate Johann Wolfgang von Goethe 



\section{Contents}

1 Introduction $\quad \mathbf{1}$

2 MR angiography of collateral arteries in a hind limb ischemia model: comparison between blood pool agent Gadomer and small contrast agent Gd-DTPA

3 Vessel-enhanced signal intensity distribution analysis for MR angiography of peripheral arteriogenesis

4 Reliability of pharmacokinetic parameters: small versus medium-sized contrast agents

5 Optimized pharmacokinetic modeling for the detection of perfusion differences in skeletal muscle with DCE-MRI: effect of contrast agent size

6 Morphological and functional monitoring of therapeutically induced neovascularization in ischemia with MRI

7 General discussion

Summary

Samenvatting

Dankwoord

Scientific publications 



\section{List of abbreviations}

$\begin{array}{ll}\text { AIF } & \text { Arterial input function } \\ \text { ASL } & \text { Arterial spin labeling } \\ \text { AUC } & \text { Area-under-curve } \\ \text { BOLD } & \text { Blood oygen level dependent } \\ \text { BPA } & \text { Blood pool agent } \\ \text { CAD } & \text { coronary artery disease } \\ \text { CE-MRA } & \text { Contrast-enhanced MRA } \\ \text { CFA } & \text { Circumflex femoral artery } \\ \text { CNR } & \text { Contrast-to-noise ratio } \\ \text { CT } & \text { Computed tomography } \\ \text { DCE-MRI } & \text { Dynamic contrast-enhanced MRI } \\ \text { DFA } & \text { Deep femoral artery } \\ \text { EES } & \text { Extracellular-extravascular space } \\ \text { FA } & \text { flip angle } \\ \text { FGF } & \text { Fibroblast growth factor } \\ \text { FOV } & \text { Field of view } \\ \text { Gd-DTPA } & \text { Gadolinium diethylenetriamine pentaacetic acid } \\ \text { GKM } & \text { Generalized kinetic model } \\ \text { Hct } & \text { Hematocrit } \\ \text { MCA } & \text { Medium-sized contrast agent } \\ \text { MCP-1 } & \text { Monocyte chemoattractant protein-1 } \\ \text { MIP } & \text { Maximum intensity projection } \\ \text { MRA } & \text { Magnetic resonance angiography } \\ \text { MRI } & \text { Magnetic resonance imaging } \\ \text { PAD } & \text { Peripheral arterial disease } \\ \text { PDGF } & \text { Platelet-derived growth factor } \\ \text { PET } & \text { Positron emission tomography } \\ \text { ROI } & \text { Region of interest } \\ \text { SCA } & \text { Small contrast agent } \\ \text { SID } & \text { Signal intensity distribution } \\ \text { SNR } & \text { Signal-to-noise ratio } \\ \text { SS-MRA } & \text { Steady-state MRA } \\ \text { TE } & \text { Echo time } \\ \text { TOF-MRA } & \text { Time-of-flight MRA } \\ \text { TR } & \text { Repetition time } \\ \text { VEGF } & \text { Vascular endothelial growth factor } \\ \text { XRA } & \text { X-ray angiogra } \\ & \end{array}$





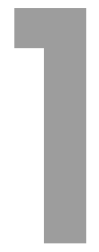

Introduction 


\subsection{Introduction}

Peripheral and coronary artery disease (PAD and CAD, respectively) are conditions characterized by arterial occlusion or stenosis due to atherosclerosis. At an early stage, they are characterized by pain during exercise. When the disease progresses, pain at rest, impaired wound healing and tissue loss may develop in patients with PAD. This end stage of PAD, referred to as critical limb ischemia, is associated with a high mortality (25\% in the first year after presentation [1]). It is also the major reason for lower limb amputation [2]. In patients with CAD, progression of the disease leads to myocardial infarction, which is a major cause of death in the industralized world. Treatment strategies for PAD and CAD aim at restoring the blood flow to the affected tissues. This can be done by creating a bypass or by placing a stent in the stenosis. However, in a large group of patients these invasive procedures are not an option or have limited effect $[3,4]$.

Currently, neovascularization therapies are under investigation as they might improve the outcome in these patients. Neovascularization refers to the formation and development of new blood vessels. It plays a role in both physiological processes such as embryonic development, wound healing and the menstrual cycle, and pathological processes such as cancer and rheumatoid arthritis. In patients with PAD and CAD, the ability to develop an increased capillary bed and to augment collateral circulation can enhance blood flow to ischemic regions and thus relieve tissue ischemia [5]. In CAD, this has a beneficial effect on infarct size [6] and ventricular function [7], and decreases mortality rates [8]. However, in most patients, the natural process is slow compared to the progression of the disease to allow sufficient restoration of the blood flow. Extensive research on the mechanisms underlying neovascularization has resulted in the identification of many vascular growth factors, which opens the ways to neovascularization therapies. 


\subsection{Mechanisms of neovascularization}

In adults, the two most common forms of neovascularization are i) the sprouting of capillaries from preexisting vessels (angiogenesis), and ii) the development of preexisting arterioles into functional collateral arteries (arteriogenesis). The two processes are triggered by different conditions and regulated by different cytokines, although some factors are involved in both processes [9]. A schematic representation of angiogenesis and arteriogenesis is provided in figure 1.1 .

\section{Angiogenesis}

The formation of capillaries, or angiogenesis is triggered by hypoxia, which upregulates the production of various cytokines, of which vascular endothelial growth factors (VEGFs) are the most important [10-12]. In response to VEGF, vascular permeability is increased, allowing plasma proteins to extravasate $[13,14]$. These proteins lay down a fibrin-rich matrix that promotes endothelial cell migration and proliferation. As a result, new vessel lumina can be formed. As a final step, the extracellular matrix is reestablished and pericytes are recruited to form mature and stable vasculature.

\section{Arteriogenesis}

A larger capillary bed in itself may not improve tissue perfusion, as the newly formed microvessels are too small to compensate for the conductance capacity of larger vessels $[9,16]$. However, it may help to stimulate the development of collateral arteries, or arteriogenesis $[17,18]$. Arteriogenesis takes place independently from tissue ischemia $[19,20]$. It is stimulated by an elevation of fluid shear stress in the preexistent arterioles caused by arterial occlusion $[18,21]$. Changes in fluid shear stress in their turn induce expression of monocyte chemoattractant protein (MCP) 1 [22, 23], which is the principal stimulator of arteriogenesis [24]. Infiltration of the vessel wall by the recruited monocytes is followed by destruction of its middle layer, the media. Activated endothelial cells subsequently upregulate growth factors associated with smooth muscle cell growth such as platelet-derived growth factor (PDGF)-B [10].

The arterioles can increase their diameter by a factor 20, and thus become fully functional arteries [25]. They do not only increase in diameter, but also in length. As the stem and re-entry zones are fixed, the vessels become tortuous and have a characteristic cork screw shape. The increased length in combination with smaller diameters makes them much more resistant to blood flow than the original large conduit artery. Consequently, the conductance is reduced and restoration of blood flow by collateral arteries is therefore limited $[18,24]$. 


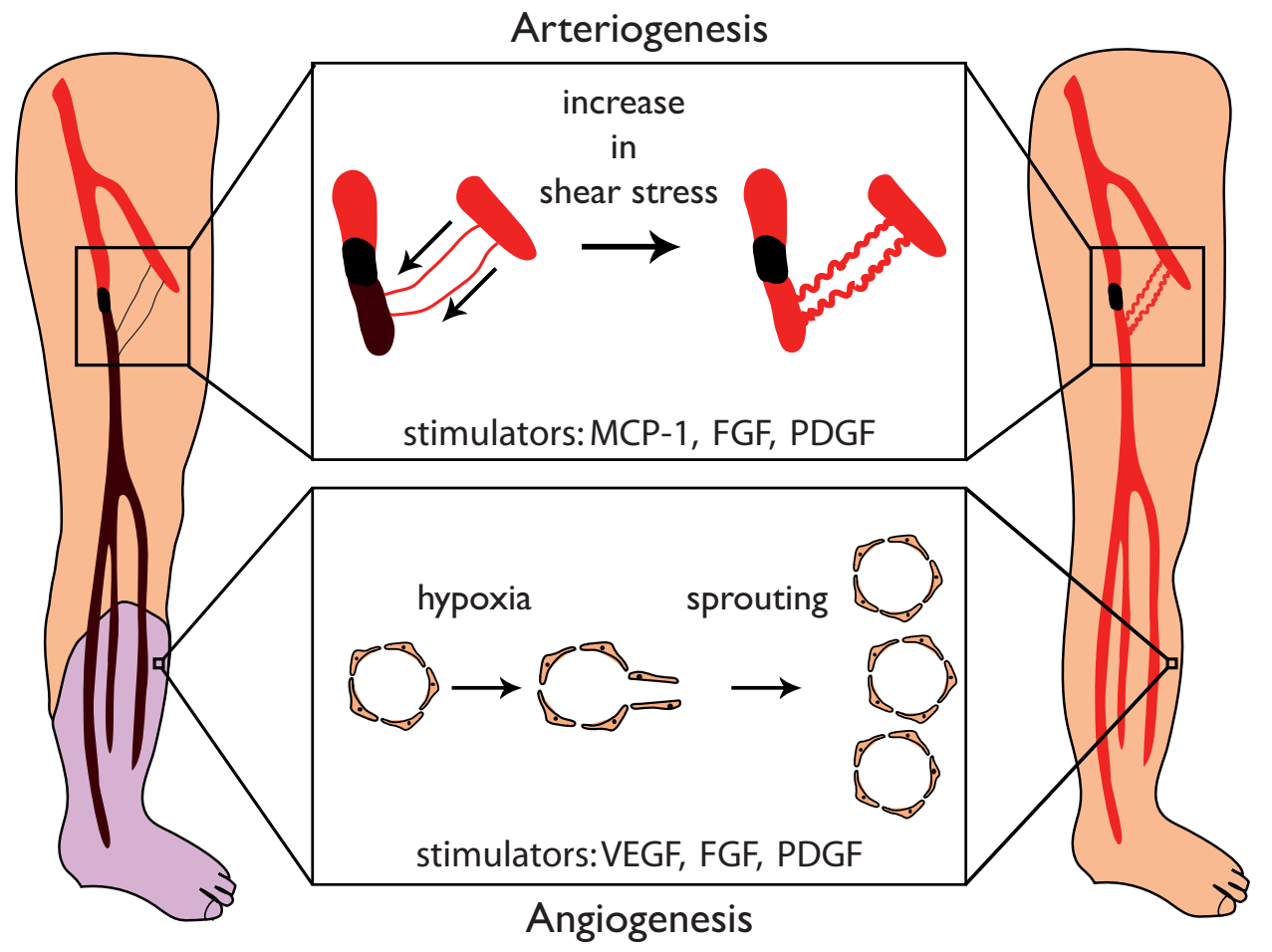

Figure 1.1: Schematic representation of neovascularization in the ischemic limb. In the thigh region, an increase in shear stress triggers arterioles bridging the arterial occlusion to develop into fully functional arteries (arteriogenesis). In the calf muscles, the occlusion causes muscle ischemia. These hypoxic conditions trigger angiogenesis, or the formation of new capillaries by means of sprouting. Growth factors involved in neovascularization are vascular endothelial growth factor (VEGFs), monocyte chemoattractant protein (MCP)-1, fibroblast growth factor (FGF) and platelet-derived growth factor (PDGF). Figure inspired by Oostendorp et al. [15]. 


\section{Neovascularization therapies}

Exogenous stimulation of neovascularization can be achieved by administration of cytokines that promote neovascularization. Extensive research has identified many of these activators (for an overview, see $[10,11,25]$ ). Some factors mainly stimulate either angiogenesis (e.g. VEGF $[12,26]$ ), or arteriogenesis (e.g. MCP-1 [24]), while others are associated with both processes (e.g. fibroblast growth factor (FGF-) 2 and PDGF-BB, which exhibit synergism when administered together [27]). Animal studies have shown the potential of growth factor therapy in animal hind limb and myocardial ischemia models [24, 27-34]. In clinical trials, FGF-2 has shown promising long-term results regarding therapy-dependent improvements in functional end points [35-38]. These therapeutic endpoints include ankle-brachial index, walking time, wound healing, and amputation rate. Although these are clinically very relevant parameters, they do not provide objective information on therapeutically-induced changes in micro- and macrovasculature [35]. The ability to measure this fundamental response to therapy may enable individual adjustment of treatment strategies, thereby improving therapeutic efficacy on an individual basis. Further development and clinical introduction would therefore benefit greatly from the availability and development of in vivo imaging techniques that can evaluate therapeutic efficacy during an earlier phase of therapy [39]. 


\subsection{Cardiovascular magnetic resonance imaging}

Of all non-invasive imaging modalities developed in the past decades, magnetic resonance imaging (MRI) arguably is the most promising for cardiovascular applications. In contrast to other techniques such as computed tomography (CT), single photon emission tomography (SPECT) and positron emission tomography (PET), it provides excellent soft tissue contrast. In addition, it does not require ionizing radiation, and can therefore be safely used for longitudinal monitoring. Compared to SPECT and PET, it has a superior spatial resolution, and MR contrast agents are considerably less nephrotoxic compared to the iodinated contrast media used for CT.

$\mathrm{MRI}$ is based on the properties of atomic nuclei in a strong magnetic field. Not all nuclei can be used, but the most abundant one in the human body, the hydrogen nucleus or proton, possesses the desired properties for MRI and is therefore most commonly used. More specifically, MR scanner settings are generally designed to measure the behavior of protons in water molecules, which are present in virtually every tissue type. Tissue contrast is based on differences in proton density and/or the size and density of molecules surrounding the water, which influence the behavior of protons in the magnetic field.

$\mathrm{MRI}$ is an extremely versatile imaging modality, as it is not limited to providing mainly anatomical (as is CT) or functional (as are SPECT and PET) data. With MRI, both anatomical and functional information can be obtained, thereby offering a comprehensive assessment of vascular changes induced by neovascularization therapies within a single imaging session.

\section{Morphological MRI}

Morphological information on the size and number of the collateral arteries can be obtained by with MR angiography (MRA). MRA techniques are based on increasing the contrast between blood vessel lumen and surrounding tissue. Vessel enhancement can be achieved with endogenous and exogenous mechanisms. Endogenous mechanisms, such as time-of-flight MRA (TOF-MRA), are generally based on the displacement of blood surrounded by stationary tissue. Exogenous techniques require administration of a contrast agent that alters the magnetic behavior of blood and are referred to as contrast-enhanced MRA (CE-MRA).

MR angiography has been used as a diagnostic tools in patients with PAD [4044] and CAD [45-47], and can serve as non-invasive alternatives for the grading and localization of stenosis and occlusions. The basic principles and subsequent advantages and disadvantages are briefly addressed in the next sections. 


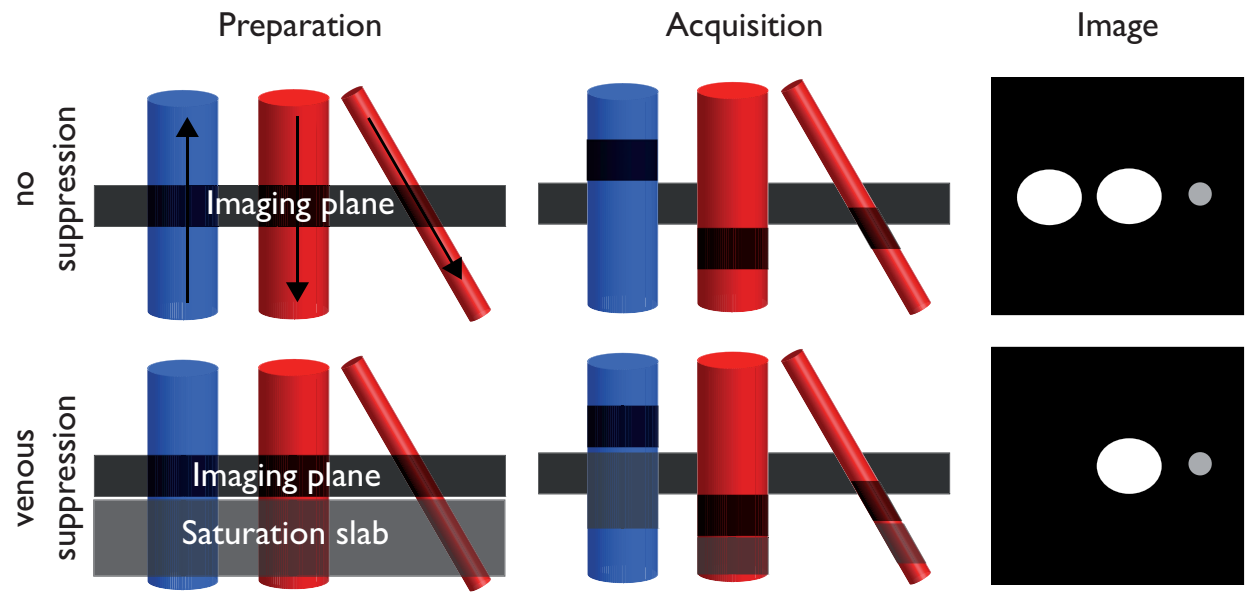

Figure 1.2: Time-of-flight MR angiography. In preparation to image acquisition, the signal in the imaging plane is suppressed. After a short period, the image is acquired. The stationary tissue is still suppressed, while the suppressed blood in vessels will have been replaced by blood with 'fresh' protons. This generates contrast between vessels and surrounding tissue. In vessels with low flow or a non-perpendicular orientation, the blood might not be completely refreshed, which results in decreased contrast. Without extra preparation steps, both arteries (in red) and veins (in blue) are enhanced (upper panels). Venous suppression can be generated by placing a saturation slab distal to the imaging plane (lower panels). In a similar fashion, arterial suppression is obtained by placing the saturation slab proximal to the imaging plane.

\section{Time-of-flight MR angiography}

TOF-MRA is a technique which uses the property of blood flow to generate vessel enhancement. In the imaging plane, all water protons are saturated by rapid repetition of RF excitation pulses. After a short delay, the original blood gets replaced by fresh, unsaturated blood from outside the imaged section, while stationary tissue remains saturated and therefore produces little signal. As a result, blood vessels appear bright compared to the background "tissue [48]. By applying a suppression slab distally or proximally to the imaging plane, selective enhancement of either arteries or veins can be achieved (see figure 1.2).

The flow-sensitivity which is the basis of the success of TOF-MRA is also the source of its main disadvantage: flow voids [41]. One cause of these apparent interruptions in the vessel course is post-stenotic turbulence. A second cause is incomplete refreshment of the blood in the imaging plane. The time allowed for replacement of saturated blood is limited because for optimal contrast, the repetition time should be kept short relative to the $T_{1}$ value of the surrounding tissue. TOFMRA is therefore less suitable for vessels that have limited flow or have trajectories positioned parallel to or at a large angle with the imaging plane (see figure 1.2). 
Another major disadvantage of TOF-MRA is the long acquisition time. To image the entire peripheral vascular tree in humans at a sufficient spatial resolution may take over an hour [41], which is clinically unacceptable because of patient discomfort, increased risk of motion artifacts and cost. The emergence of contrast agents therefore almost completely replaced TOF-MRA with CE-MRA in the clinical setting, but low resolution TOF-MRAs are clinically used as a prescan to plan the subsequent CE-MRA acquisition.

\section{Contrast-enhanced MR angiography}

In CE-MRA, vascular enhancement is achieved by intravenous administration of a contrast agent [49]. The largest group of contrast agents consists of paramagnetic gadolinium $\left(\mathrm{Gd}^{3+}\right)$ chelates. These agents cause a reduction of the $T_{1}$ relaxation rate that depends on the concentration and $T_{1}$ relaxivity properties of the contrast agent. With $3 \mathrm{D} T_{1}$-weigthed gradient echo sequences with short $\mathrm{TR}$, the background signal becomes saturated while the vessels filled with contrast agent appear bright. The main advantage of this technique over TOF-MRA is that relatively high spatial resolutions can be achieved within short acquisition times [41]. CE-MRA is less flow-sensitive and consequently does not suffer from flow voids.

Image acquisition is preferably performed during the first arterial passage of the contrast agent bolus. During this period, the arterial contrast agent concentration is highest, providing maximal $T_{1}$ shortening while the venous concentration is still low, which results in selective arterial enhancement. To maximally profit from the advantages of so-called first-pass MRA, the acquisition should be properly timed. The bolus arrival time varies among patients. In the femoral artery, for example, bolus arrival times between 13 and 37 seconds have been reported [50]. The optimal acquisition timing therefore needs to be determined for each individual patient. To this end, various methods have been developed. Injection of a small test bolus during acquisition of a test plane every 1 - 2 seconds is effective, but requires extra time for set-up and analysis [51]. Another method is to start the acquisition based on the real-time arrival of the contrast agent bolus by triggering [52-54] or MR fluoroscopy $[55,56]$.

\section{Challenges in MR angiography of collateral arteries}

The small caliber and tortuous shape of collateral arteries set high standards to MR acquisition. In CE-MRA, the risk of improper timing is especially high in patients with $P A D$, in whom correct acquisition timing is hindered by the complex structure of the peripheral vascular tree associated with PAD. The presence of stenoses and a network of small collateral arteries may cause unpredictable bolus arrival times in the run-off vessels. Consequently, bolus arrival times as measured in relatively large arteries may 


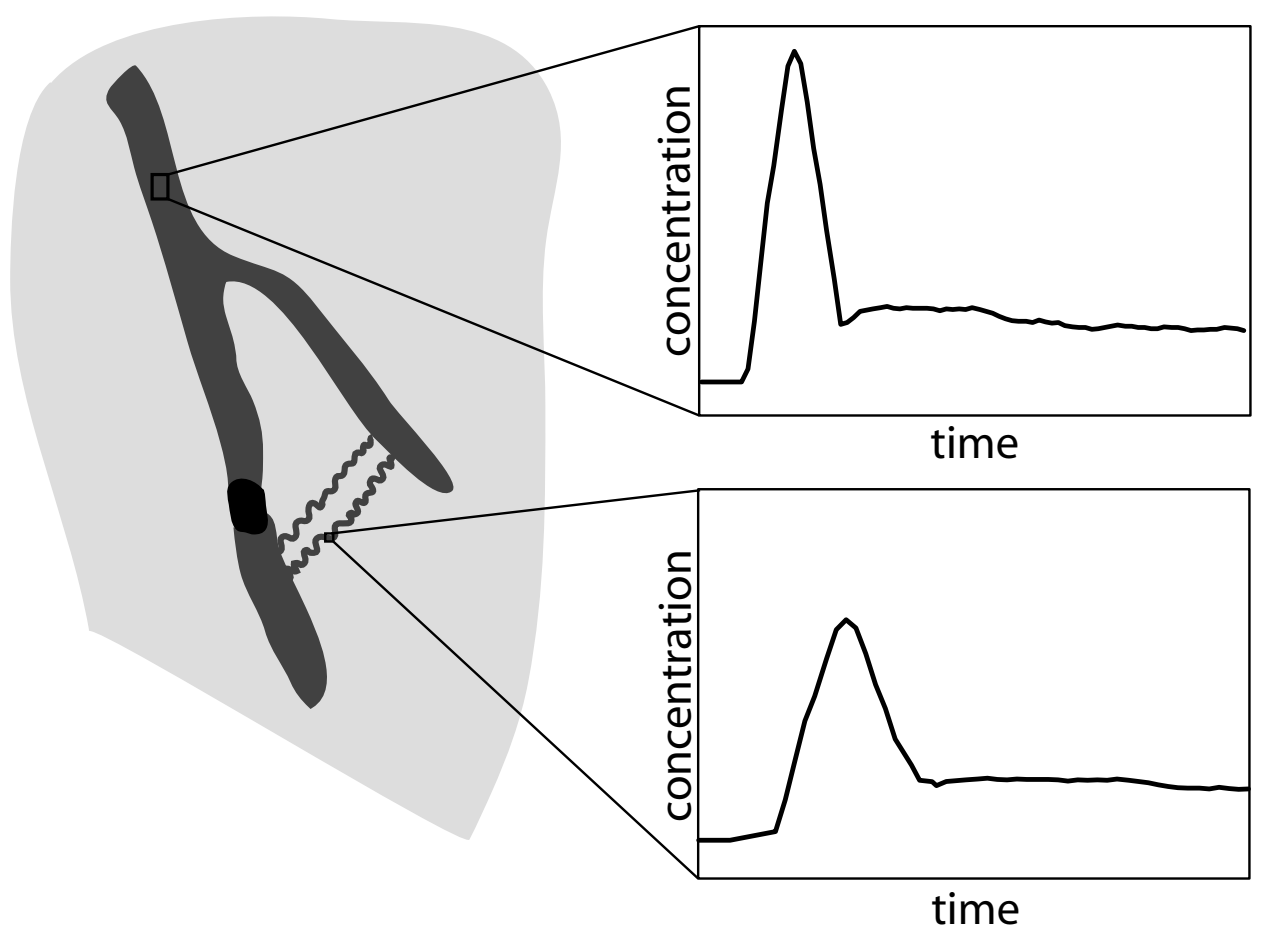

Figure 1.3: Uncertainty in bolus arrival time associated with a collateral network. The smaller diameter and increased length of collateral arteries may deform the shape of the plasma concentration time curve. This may result in a delayed arrival as well as a dispersion of the bolus in the collateral arteries compared to the large supplying artery. Plasma concentration time curves are based on data acquired in the aorta [58].

not be representative to the arrival times in the more distal vasculature [57] (see figure 1.3).

When image acquisition is limited to the first pass of the contrast agent, a constraint is imposed on the duration of the acquisition and, consequently, on the achievable spatial coverage and resolution. However, after the first pass, the concentration of small contrast agents such as Gd-DTPA decreases rapidly, as they leak easily through the gaps between the endothelial monolayer that constitutes the capillary vessel wall. By using larger contrast agents, which remain mainly intravascular due to their higher molecular weight, prolonged vascular enhancement can be achieved [59], while the concentration in the surrounding tissue remains low. This enables acquisition in the post-bolus or steady-state phase. Due to its longer acquisition time, steady-state MRA is less sensitive for timing uncertainties compared to first-pass MRA, which has been shown to improve the visualization of severe stenoses with low perfusion [57], at the cost of concurrent venous enhancement. 
Quantification of the arteriogenic response based on MR angiograms is not straightforward. Merely counting the number of collateral arteries provides little information on changes in vessel size and physiological impact. Moreover, visualization of complete trajectories is challenging due to the ultra-small collateral artery calibers $(<0.5 \mathrm{~mm})$. This makes manual identification based on the Longland criteria, which requires identification of the stem, mid- and re-entry zone [60], time consuming and observer dependent. The availability of (semi-) automated analysis strategies would therefore provide more objective measures.

\section{Functional MRI}

The main goal of therapeutic neovascularization is to improve distal tissue perfusion. Fuchs et al. [61] demonstrated that changes in morphology do not necessarily result in changes in tissue perfusion, which stresses the need for functional measurements [62]. MRI provides several techniques for perfusion measurements. Examples of noncontrast-enhanced methods include blood oxygenation level-dependent (BOLD) MRI, which can be used to determine the perfusion capacity of the lower limb musculature during reactive hyperemia [63-65], and arterial spin labeling or ASL $[66,67]$. A promising contrast-enhanced technique is dynamic contrast-enhanced (DCE) MRI. This technique, which is based on the extravasation of a contrast agent out of the capillaries into the extracellular extravascular space (EES), is already an established diagnostic method in oncology [68-70]. Additionally, it can be used for monitoring anti-cancer therapy [71-74]. More recent studies also show promising results in the evaluation of muscle ischemia [75-78].

\section{Dynamic contrast-enhanced MRI}

DCE-MRI is based on quantifying the passage and extravasation of a contrast agent from the capillaries into the extracellular extravascular space (EES). The time course of contrast agent concentration in the tissue $C_{t}(t)$ is governed by tissue properties such as volume fraction of the EES, and microvascular properties such as flow, vessel permeability, vessel surface and plasma fraction. Perfusion-related parameters can be taken directly from $C_{t}(t)$ or derived with a pharmacokinetic model. Modelfree parameters include area-under-curve (AUC), time-to-peak and wash-in gradient. Model-free approaches are relatively independent of injection protocols [79] and highly reproducible [80]. However, since the underlying physiological mechanisms remain undefined, the interpretation of model-free parameters is ambiguous and depends on the method and contrast agent properties.

Physiologically more relevant parameters can be derived with pharmacokinetic modeling. To this end, several models with varying complexity $[70,81-84]$ have been developed to describe the relation between $C_{t}(t)$ and plasma concentration time curve $C_{p}(t)$ in terms of extravasation rate $K^{\text {trans }}$, fraction of the EES $v_{e}$ and/or 
plasma fraction $v_{p}$ (see figure 1.4). The physiological interpretation of $K^{\text {trans }}$ is not straightforward, as it is related to both flow $(F)$ and permeability surface area product $(P S)$ by $K^{\text {trans }}=F\left(1-e^{\frac{-P S}{F}}\right)$ [83]. The degree to which $F$ and $P S$ contribute is determined by their ratio. If $P S$ is high (i.e. the contrast agent is small compared to the endothelial gaps) compared to the flow, extravasation is limited by the supply of contrast agent, and consequently by flow. In this case, which generally applies to small contrast agents, $K^{\text {trans }}$ mainly reflects microvascular flow, resulting in a high correlation between $K^{\text {trans }}$ and perfusion as measured with microspheres $[85,86]$.

\section{Challenges in DCE-MRI}

For reliable estimation of pharmacokinetic parameters, the tissue concentration time curve needs to be sampled at an appropriate sampling rate. Most DCE-MRI studies have used clinically approved small contrast agents such as gadopentetate dimeglumine (Gd-DTPA). However, these agents extravasate rapidly and require fast imaging protocols [87-89]. DCE-MRI would therefore benefit from the use of larger contrast agents, which have reduced leakage rates [90]. Such contrast agents would allow longer dynamic acquisition times, which could be employed to improve image quality by increased signal-to-noise ratio, spatial resolution, and/or spatial coverage.

The rate of extravasation of a large contrast agent is mainly limited by the size and density of endothelial gaps (expressed in PS). In this case, differences in extravasation rate are more likely to reflect differences in capillary permeability than variations in flow. This is an advantage in oncology [74,91-93], where increased vascular permeability caused by angiogenesis is associated with tumor malignancy. For tissue perfusion imaging, extravasation should not be exclusively permeabilitylimited, but also be sensitive to perfusion differences. The use of medium-sized contrast agent, which are small enough to be sensitive to flow but large enough to allow longer dynamic acquisition times might be ideal for muscle perfusion measurements. 


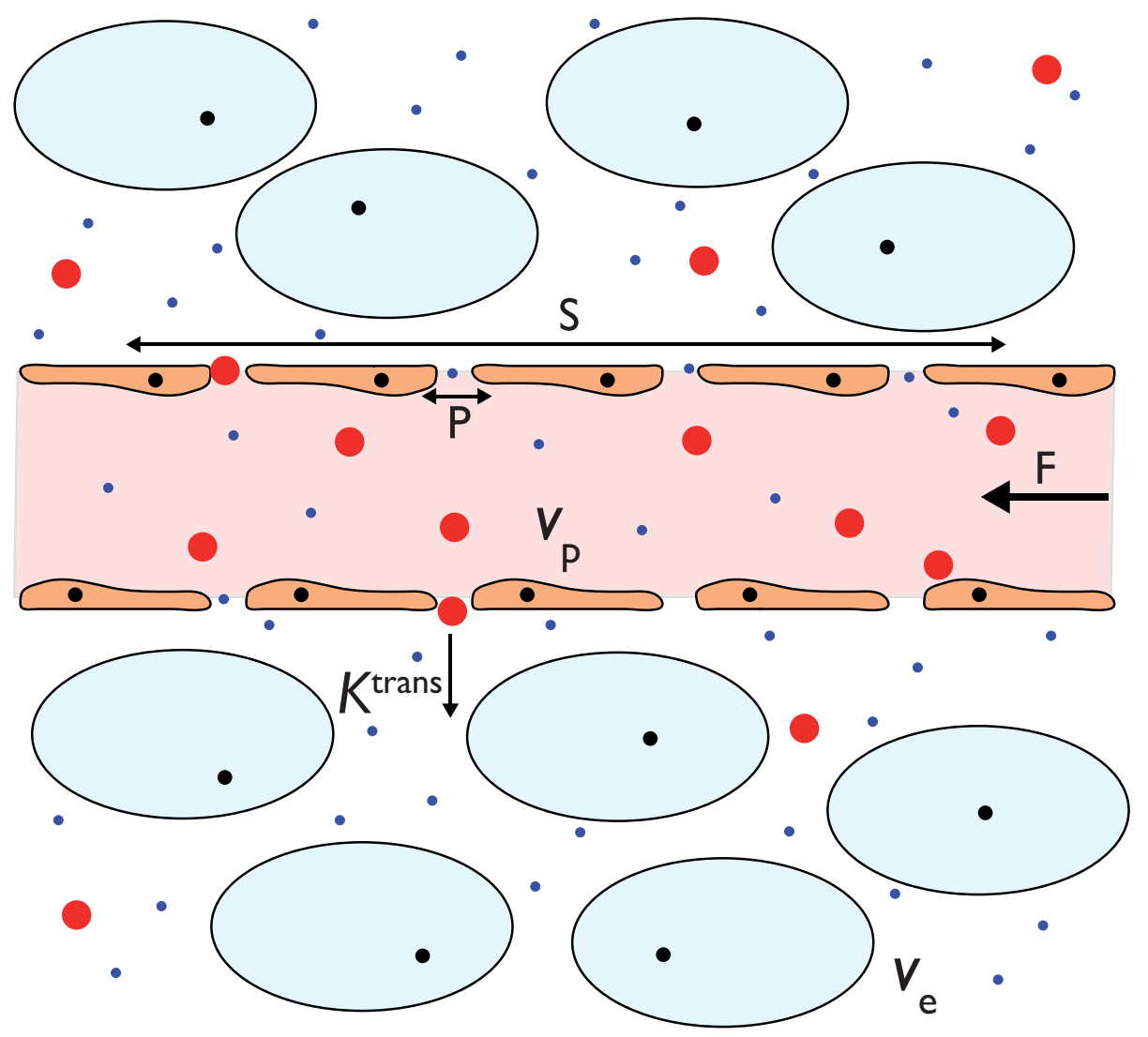

Figure 1.4: Contrast agent extravasation and pharmacokinetic parameters. Contrast agents can extravasate from the blood plasma (with volume fraction $v_{p}$ ) through the gaps between the endothelial cells making up the capillary vessel wall into the extracellular extravascular space (with volume fraction $v_{\mathrm{e}}$ ). For small contrast agents (in blue) which leak easily through the gaps, the rate of extravasation $K^{\text {trans }}$ is mainly dominated by contrast agent supply, and consequently by the microvascular flow $F$. For larger contrast agents (in red), the size of the contrast agent size is comparable to the size of the endothelial gaps. In this case, $K^{\text {trans }}$ is limited by the product of contrast agent permeability $P$, which is proportional to the number and relative size of the gaps, and the capillary surface area $(S)$. 


\subsection{Outline thesis}

This thesis discusses several morphological and functional MR techniques that can be used for the monitoring of neovascularization therapies.

Chapter 2 compares a blood pool agent with a small contrast agent with respect to the visualization of collateral arteries with steady-state MR angiography in a rabbit hind limb ischemia model.

In Chapter 3, an automated analysis method for the quantification of collateral artery development based on the distribution of pixel signal intensities is introduced and tested in a rat hind limb ischemia model.

Chapter 4 addresses the reliability of pharmacokinetic parameter estimation. Monte Carlo simulations were performed to investigate the influence of DCE-MRI measurement settings for a medium-sized and small contrast agent.

Subsequently, Chapter $\mathbf{5}$ discusses the optimization of DCE-MRI data analysis for both a medium-sized and small contrast agent, and investigates the sensitivity for skeletal muscle perfusion differences in a rabbit hind limb ischemia model.

In Chapter 6, the potential of combined functional and morphological MR techniques for the in vivo evaluation of therapeutically induced neovascularization is studied in a rat hind limb ischemia model. DCE-MRI, MRA and $T_{2}$-weighted measurements were used to obtain parameters reflecting arteriogenesis, angiogenesis and their effects on muscle perfusion at multiple time points.

In the General Discussion presented in Chapter 7, the main conclusions of this thesis are drawn, discussed and placed in a broader perspective. 

MR angiography of collateral arteries in a hind limb ischemia model: comparison between blood pool agent

Gadomer and small contrast agent

Gd-DTPA 


\section{Abstract}

The objective of this study was to compare the blood pool agent Gadomer with a small contrast agent for the visualization of ultra-small, collateral arteries (diameter $<1 \mathrm{~mm}$ ) with high resolution steady-state MR angiography (SS-MRA) in a rabbit hind limb ischemia model.

Ten rabbits underwent unilateral femoral artery ligation. On days 14 and 21 , high resolution SS-MRA (voxel size $0.49 \times 0.49 \times 0.50 \mathrm{~mm}^{3}$ ) was performed on a 3 Tesla clinical system after administration of either Gadomer (dose: $0.10 \mathrm{mmol} / \mathrm{kg}$ ) or a small contrast agent (gadopentetate dimeglumine (Gd-DTPA), dose: 0.20 $\mathrm{mmol} / \mathrm{kg}$ ). All animals received both contrast agents on separate days. Selective intra-arterial $\mathrm{x}$-ray angiograms (XRAs) were obtained in the ligated limb as a reference. The number of collaterals was counted by two independent observers. Image quality was evaluated with the contrast-to-noise ratio (CNR) in the femoral artery and collateral arteries.

CNR for Gadomer was higher in both the femoral artery (Gadomer: $73 \pm 5$ (mean \pm SE); Gd-DTPA: $40 \pm 3 ; p<0.01$ ) and collateral arteries (Gadomer: $18 \pm 4$; GdDTPA: $9 \pm 1 ; p=0.04)$. Neither day of acquisition nor contrast agent used influenced the number of identified collateral arteries ( $p=0.30$ and $p=0.14$, respectively). An average of $4.5 \pm 1.0$ (day 14, mean \pm SD) and $5.3 \pm 1.2$ (day 21) collaterals was found, which was comparable to XRA ( $5.6 \pm 1.7$, averaged over days 14 and 21 ; $p>0.10$ ). Inter-observer variation was $24 \%$ and $18 \%$ for Gadomer and Gd-DTPA, respectively.

In conclusion, blood pool agent Gadomer improved vessel conspicuity compared to Gd-DTPA. Steady-state MRA can be considered as an excellent non-invasive alternative to intra-arterial XRA for the visualization of ultra-small collateral arteries. 


\subsection{Introduction}

Therapeutic stimulation of the development of collateral arteries from pre-existent arterioles (arteriogenesis) seems attractive as an alternative or adjuvant treatment for patients with peripheral arterial occlusive disease [27, 33, 94]. Further progress in the development of such treatment strategies relies strongly on the availability of non-invasive imaging methods that are able to evaluate the efficacy of treatment in terms of vascular changes at an early stage, even before clinical benefit can be noticed.

Therapeutic efficacy can be evaluated functionally, i.e. by measuring perfusion recovery-related parameters in tissue distal to the vascular lesion, or morphologically, i.e. by quantifying the number and size of the collaterals formed. Contrast-enhanced MR angiography (CE-MRA) has already proven to be a promising non-invasive tool to visualize collateral arteries in various parts of the body [95-98]. Preferably, CE-MRA images are acquired during the first pass of the bolus, when the arterial concentration is highest and veins are not yet enhanced. However, visualization of small peripheral collateral arteries (diameter $<1 \mathrm{~mm}$ ) requires both a very high spatial resolution and a large spatial coverage. Meeting these requirements within the duration of the first pass period is a challenge. Moreover, correct timing of the first-pass acquisition is problematic in patients with severe stenoses and an extensive network of collateral arteries, whose filling occurs very slowly and varies considerably among and within patients $[56,99]$.

An alternative is to acquire data during the post-bolus equilibrium phase or steady state, which permits longer acquisition times and therefore a higher spatial resolution. Due to its longer acquisition time, steady-state MRA (SS-MRA) is less sensitive for timing uncertainties than first-pass MRA (FP-MRA), as demonstrated for the visualization of arterial stenoses in patients with peripheral arterial occlusive disease [57]. To obtain sufficient signal from the arteries, a high arterial contrast agent concentration over the entire duration of the acquisition is desired. However, most currently available contrast agents are small (e.g. gadopentetate dimeglumine (Gd-DTPA), molecular weight: $0.5 \mathrm{kDa}$ ) and extravasate rapidly after injection, resulting in both a decrease in arterial signal intensity and an increase in background enhancement. These problems may be solved by the use of larger contrast agents which remain mainly intravascular, thereby providing prolonged vascular enhancement [59]. Moreover, these so-called blood pool agents (BPAs) generally have a higher $T_{1}$ relaxivity compared to small contrast agents because of their size [100], allowing reduction of contrast agent dose without compromising signal-to-noise characteristics [47, 101-104].

Various types of types of gadolinium-based BPAs have been developed. One group consists of small BPAs that non-covalently bind to plasma proteins, with the albumin-binding gadofosveset trisodium as its principal, clinically approved example [105]. A second group consists of medium-sized blood pool agents such as P792 
$[106,107]$ and Gadomer [108], which are currently under consideration for use in humans. Their value in diagnostics and pretreatment assessment of peripheral and coronary arterial disease has been demonstrated $[57,59,109-111]$, yet their potential in the visualization of ultra-small arteries required for the evaluation of collateral artery formation remains to be explored.

In this study, we compared the blood pool agent Gadomer to the small contrast agent Gd-DTPA for the visualization of small collateral arteries with steady-state contrast-enhanced MRA in a standardized rabbit hind limb ischemia model exhibiting arteriogenesis. For both contrast agents, image quality characteristics and number of visible collaterals were determined and compared to intra-arterial XRA. 


\subsection{Materials and Methods}

\section{Animal model}

This study was approved by the Maastricht University animal ethics committee (approval ID 06-022). Ten male New Zealand White rabbits (weight: approximately $3 \mathrm{~kg}$ ) were included. Occlusion of the right femoral artery was realized by placing ligations $1 \mathrm{~cm}$ below the branch of the circumflex femoral artery (CFA) and $1 \mathrm{~cm}$ above the bifurcation of the popliteal and tibial arteries.

For all procedures, anesthesia was induced by intramuscular injection of a combination of ketamine hydrochloride $(50 \mathrm{mg} / \mathrm{kg}$ body weight) and xylazin $(8 \mathrm{mg} / \mathrm{kg}$ body weight). During the procedures, the rabbits were intubated and ventilated with isoflurane $(2-3 \%)$ and oxygen. Post-operative analgesia was provided during three days by daily intramuscular injections of buprenorphine $(0.09 \mathrm{mg} / \mathrm{kg}$ body weight $)$. All invasive procedures were performed under sterile conditions. After the last MR measurements a lethal dose of pentobarbital $(1 \mathrm{~mL} / \mathrm{kg}$ body weight) was administered intravenously.

\section{X-ray angiography}

On days 14 and 21 post ligation, XRA series of anterior-posterior projections were recorded using a portable $x$-ray system (BV Pulsera, Philips Medical Systems, Best, The Netherlands). In-plane resolution was $0.2 \times 0.2 \mathrm{~mm}^{2}$, tube voltage was $72 \mathrm{kV}$ and frame rate was $12 \mathrm{~s}^{-1}$. An intra-arterial catheter was inserted into the left carotid artery and positioned in the iliac artery of the ligated limb, where a $5 \mathrm{~mL}$ bolus of non-ionic iodine contrast agent (Omnipaque, Amersham Health, Eindhoven, The Netherlands, $240 \mathrm{mg}$ iodine $/ \mathrm{mL}$ ) was injected at $5 \mathrm{~mL} / \mathrm{s}$, followed by a saline flush.

\section{MR angiography}

Within one hour after XRA, the animals were imaged in supine position on a clinical 3.0 Tesla MRI system equipped with a 5-element phased-array cardiac coil (Philips Medical Systems, Best, The Netherlands). The rabbits were placed in a custommade fixating box to standardize animal positioning.

The steady-state MRA (SS-MRA) protocol consisted of a $T_{1}$-weighted spoiled fast gradient echo sequence. Field-of-view (FOV) in the cranio-caudal read-out direction was $250 \mathrm{~mm}$ and rectangular FOV was $80 \%$. Parallel imaging acceleration (SENSE) factor in the right-left direction was 2. Acquisition was started after a delay of two minutes. Matrix size was $512 \times 410$ with 282 overlapping coronal slices (slice 
thickness $0.5 \mathrm{~mm}$ ). Measured voxel size was $0.49 \times 0.49 \times 0.50 \mathrm{~mm}^{3}$. TR/TE/FA were $10.5 \mathrm{~ms} / 2.7 \mathrm{~ms} / 25^{\circ}$. Acquisition time was 5 minutes.

\section{Contrast agents}

To all animals both a small extravascular agent (Gd-DTPA) and a medium-sized blood pool agent (Gadomer) were administered. Gadomer (Schering Pharma AG, Berlin, Germany) is a dendritic gadolinium chelate, containing $24 \mathrm{Gd}^{3+}$ ions. It has a molecular weight of $17 \mathrm{kDa}$. Gd-DTPA and Gadomer were administered in a randomized order on separate days. The contrast agent (vial concentration 0.5 $\mathrm{M}$, injected volume approximately $0.3 \mathrm{~mL}$ or $0.6 \mathrm{~mL}$, for Gadomer and Gd-DTPA, respectively) was injected at $0.05 \mathrm{~mL} / \mathrm{s}$ into an ear vein, followed by $2 \mathrm{~mL}$ saline flush injected at the same rate.

The contrast agent dose was selected by estimating signal enhancement during the course of SS-MRA acquisition based on a biexponential approximation of contrast agent concentration determined in a previous study [112] for a range of doses. A full description of the procedure is found in Appendix I. The optimal dose for Gadomer was $0.10 \mathrm{mmol} \mathrm{Gd} / \mathrm{kg}$. For Gd-DTPA, maximum signal enhancement was obtained with a dose of $0.50 \mathrm{mmol} \mathrm{Gd} / \mathrm{kg}$. However, this was considered too high, and the difference among doses in the range of $0.20-0.50 \mathrm{mmol} G d / \mathrm{kg}$ was small. We therefore selected the lowest dose in this range $(0.20 \mathrm{mmol} / \mathrm{kg})$. For these doses, the average concentration during image acquisition is $0.8 \mathrm{mM}$ for Gadomer and 1.3 $\mathrm{mM}$ for Gd-DTPA.

\section{Image analysis}

\section{Image processing}

From the dynamic XRA image series a maximum intensity projection (MIP) was calculated in MATLAB (The MathWorks, Natick, MA, version R2007b) over the time frames that exhibited arterial enhancement. The resulting images were used for collateral identification. The MR angiograms were viewed in the image processing application OsiriX (version 3.7), using the source images for signal intensity measurements. For collateral identification, coronal maximum intensity projections over a limited range of slices (partial MIPs) were used. The thickness and location of the slab, as well as the display contrast and brightness levels could be adjusted in real time to obtain optimal depiction of the collateral trajectories, and varied depending on image quality and vascular anatomy. 


\section{Collateral quantification}

The number of collaterals was counted on the partial MIP by two independent observers using the Longland definition [60], which requires identification of the stem, mid- and re-entry zone. A distinction was made between collaterals stemming from the deep femoral artery (DFA) and circumflex femoral artery (CFA) [113]. To evaluate whether the same collateral arteries were identified between measurements, the observers provided a schematic representation of the course of the identified collaterals, including the approximate position of the stem and re-entry point.

Arteries were separated from veins based on their size, location, and branching point. Due to evident differences in image quality, the observers were not blinded for contrast agent.

\section{Image quality}

Signal intensities (SI) were measured in the femoral artery (proximal of the occlusion), a collateral artery stemming from the DFA, and in muscle tissue surrounding the collateral. Noise was estimated by calculating the standard deviation of a relatively homogeneous part of adjacent muscle tissue ( $\left.\mathrm{SD}_{\text {muscle }}\right)$. From these data signal-tonoise (SNR) and contrast-to-noise (CNR) ratios were calculated according to:

$$
\mathrm{SNR}=\frac{\mathrm{SI}_{\text {vessel }}}{\mathrm{SD}_{\text {muscle }}} \quad \text { and } \quad \mathrm{CNR}=\frac{\mathrm{SI} \text { vessel }-S I_{\text {muscle }}}{\mathrm{SD}_{\text {muscle }}}
$$

\section{Statistics}

Interobserver agreement of the collateral artery counting was assessed with a betweenobserver coefficient of variation. The difference in number of collaterals between XRA and MRA was tested using a paired Wilcoxon signed rank test. A repeated analysis of variance (ANOVA) was used to test whether day of acquisition or contrast agent used (for MRA) had an influence on the number of identified collateral arteries. Independent sample two-sided Student's $t$-tests were performed to test the difference in SNR and CNR between Gadomer and Gd-DTPA for the femoral artery and a collateral stemming from the DFA in ligated limb. Effects were considered significant for $p<0.05$. 


\subsection{Results}

\section{Image quality}

On the steady-state MR angiograms, both arteries and veins were enhanced, but the spatial configuration of the vessels and the high spatial resolution of the angiograms allowed distinction of arteries from veins (see figure 2.1). For Gadomer, thick-slab MIPs (thickness $>10 \mathrm{~mm}$ ) provided the best overview of the vascular system, but for Gd-DTPA these images appeared too blurred to be of use. On thin-slab MIPs (thickness $<5 \mathrm{~mm}$ ), the advantage of Gadomer was less pronounced. Although the femoral and collateral arteries appeared more conspicuous with Gadomer, contrast between artery and background tissue was sufficiently high to identify the complete trajectories of the collaterals for both contrast agents.

SNR was higher in both the femoral and collateral arteries for Gadomer compared to Gd-DTPA (figure 2.2). This improvement was significant in the femoral artery $(p<0.01)$, and had the same, yet statistically non-significant trend in the collateral arteries $(p=0.10)$. CNR was significantly higher in both femoral and collateral arteries when Gadomer was used (femoral artery: $p<0.01$; collateral artery: $p=0.04$ ). ROI size was typically 10 voxels in the femoral artery and 3 voxels in the collateral artery. Noise and background signal was measured over an area of approximately 3 $\mathrm{cm}^{2}$.

\section{Collateral quantification}

Table 2.1 lists the results of the collateral quantification. On day 14, an average of 4.9 and 4.3 collaterals was found for Gadomer and Gd-DTPA, respectively, and on day 21 an average of 5.8 (Gadomer) and 4.5 (Gd-DTPA) arteries were identified. Analysis of variance revealed that neither day of acquisition nor contrast agent used influenced the number of identified collateral arteries $(p=0.30$ and $p=0.14$, respectively). Although the animals were positioned in a custom-made fixating box,

Figure 2.1 (facing page): Contrast-enhanced MR angiograms with Gadomer and Gd-DTPA Partial maximum intensity projections (MIPS) in the anterior-posterior direction of steadystate MR angiograms (SS-MRA) for Gadomer (left column) and Gd-DTPA (right column). On the thin-slab MIPs (thickness 3 - 4 slices, panels a and b), collaterals could be discerned for both contrast agents. Thick slab MIPs (> 20 slices; panels $c$ and d) show superior depiction of vessels with Gadomer. For Gd-DTPA, the images were blurred. Panels e and $f$ show the corresponding $x$-ray angiograms (XRA). 1: femoral artery; 2 : circumflex femoral artery; 3: deep femoral artery; 4: tibial artery; 5: popliteal artery; 6: popliteal vein; 7 : iliac artery; 8: iliac vein. * indicate collaterals. The ligation is indicated with the arrow in the XRA images. All images were acquired on day 21. 
Gadomer
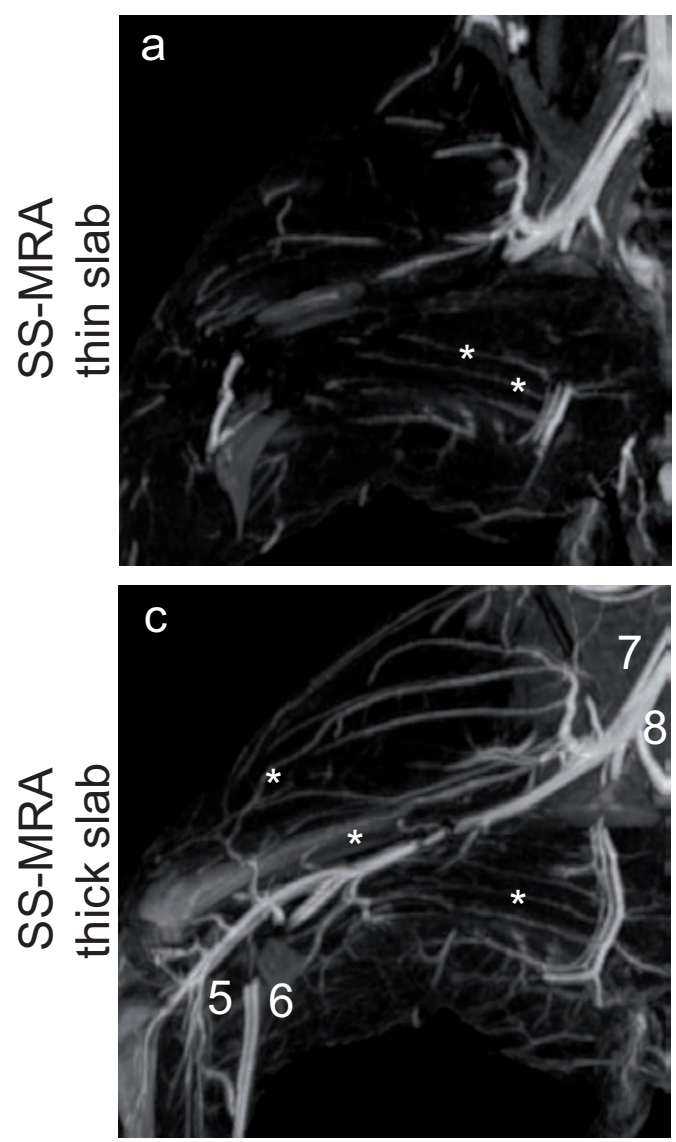

Corresponding XRA

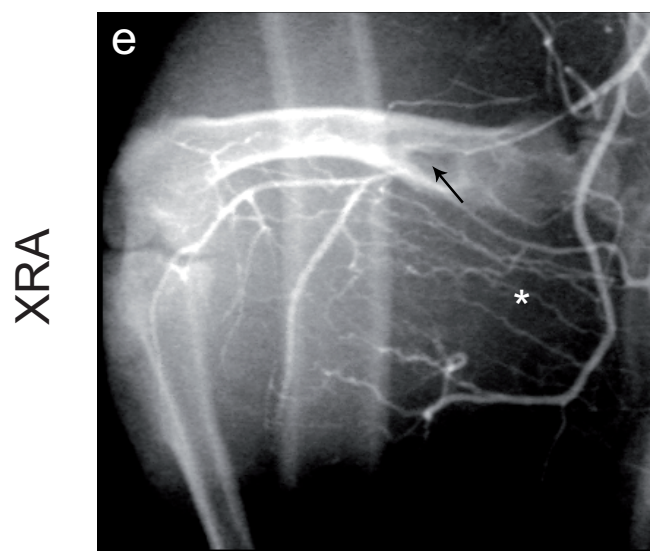

Gd-DTPA
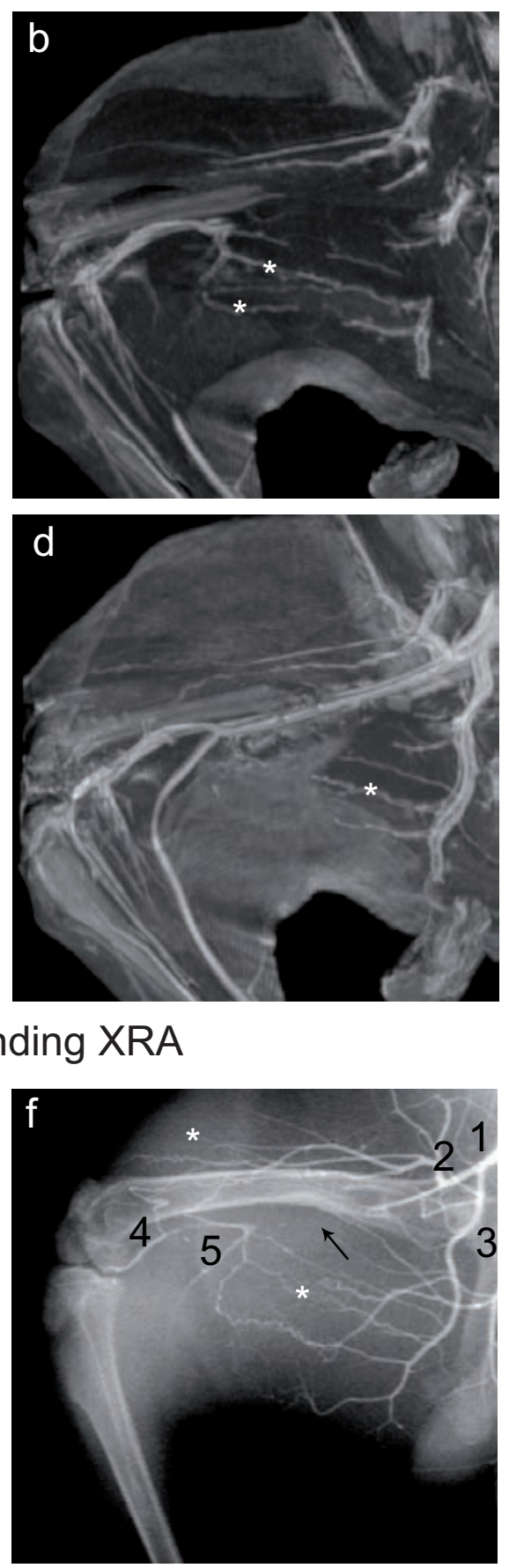

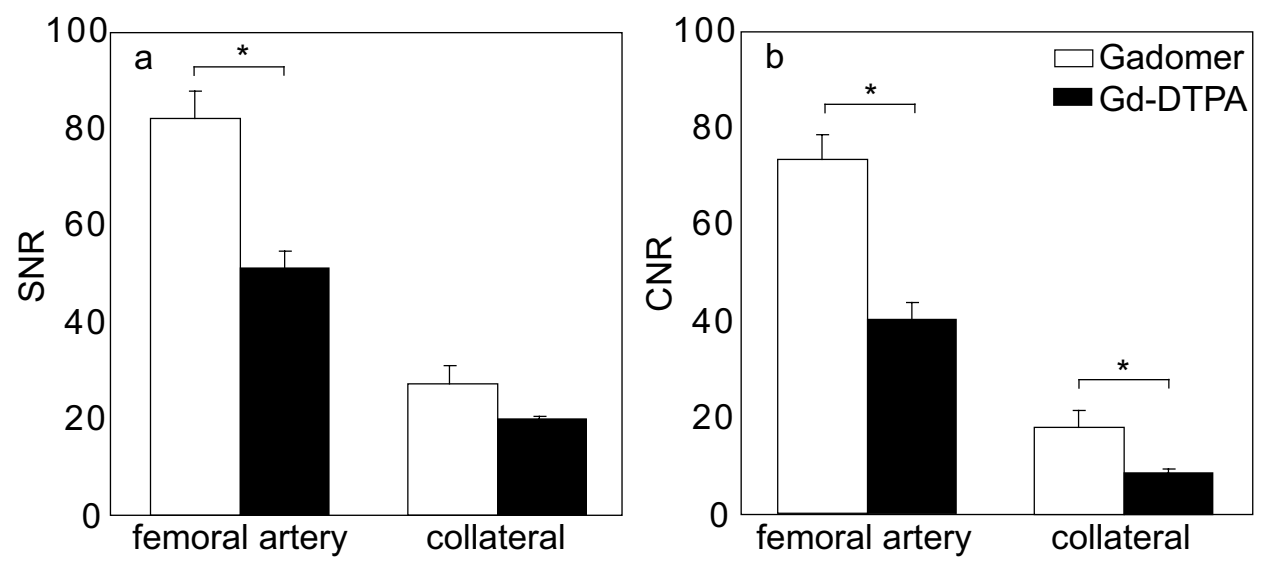

Figure 2.2: SNR and CNR in femoral and collateral artery Mean signal-to-noise (SNR, panel a) and contrast-to-noise ratio (CNR, panel b) in the femoral artery and a collateral artery originating from the deep femoral artery in the steady-state MR angiograms for Gadomer (white) and Gd-DTPA (black). Error bars indicate standard error of the mean. Significant differences between contrast agents $(p<0.05)$ are indicated with *. Data were averaged over days 14 and 21 post ligation.

it was not feasible to perform 1-to-1 comparison of the collaterals found with either contrast agent due to difficulties in determining the exact course of the collateral arteries. Inter-observer variation in the number of identified collaterals on SS-MRA was $24 \%$ and $18 \%$ for Gadomer and Gd-DTPA, respectively, meaning that the difference between observers is approximately one collateral artery. Identification of the exact points of branching and re-entering appeared to be highly subjective, and no conclusions could be drawn from the schematic vessels courses.

The number of collateral arteries found with XRA increased between days 14 and 21 (day 14: $5.1 \pm 1.7$ (mean \pm SD); day 21: $6.3 \pm 1.8 ; p=0.04$ ). The number of collateral arteries found with XRA was equal to the number found with MRA $(p=0.08)$. For $\mathrm{XRA}$, most collaterals originated from the deep femoral arteries (120 out of 172 or $70 \%$ ), whilst for MRA only $50 \%$ (77 out of 153) stemmed from the DFA. 


\subsection{Discussion}

\section{Current findings}

To our knowledge, this is the first study that directly compares the efficacy of the blood pool agent Gadomer with Gd-DTPA for the visualization of ultra-small collateral arteries. The number of collaterals identified on the steady-state images was equal for the two contrast agents, and closely mirrored the number of collaterals identified on high resolution invasive $\mathrm{X}$-ray angiograms. Contrast enhancement in collateral arteries in terms of CNR and therefore vessel conspicuity, was superior for Gadomer compared to Gd-DTPA.

Table 2.1: Number of identified collateral arteries Number of identified collaterals (mean \pm SD) on steady-state MR angiograms (MRA), averaged over two observers, with Gadomer and gadopentetate dimeglumine (Gd-DTPA) and intra-arterial x-ray angiograms (XRA) on days 14 and 21 . * indicates an significant increase in number of collaterals $(p<0.05)$.

\begin{tabular}{cll}
\hline & Day 14 & Day 21 \\
\cline { 2 - 3 } Gadomer & $4.9 \pm 1.1$ & $5.8 \pm 1.3$ \\
Gd-DTPA & $4.3 \pm 0.9$ & $4.5 \pm 1.0$ \\
XRA & $5.1 \pm 1.7$ & $6.3 \pm 1.8 *$ \\
\hline
\end{tabular}

\section{Blood pool agents versus small contrast agents}

The use of Gadomer resulted in a considerably higher contrast between collateral arteries and background compared to Gd-DTPA in the SS-MRA images. Because of this improved image quality, thick-slab MIPs could be used to get a better overview of the vessel trajectories, which notably facilitated the localization of collateral arteries, thus making it less time-consuming. For Gd-DTPA, the value of these thick-slab MIPs was limited, because background enhancement became problematic for slabs thicker than approximately 8 slices $(5 \mathrm{~mm}$ ). Moreover, thick-slab MIPs may suggest connections between vessels which are in fact crossings of different vessels. Therefore, to follow the trajectory of the collateral artery from stem to re-entry zone, thinslab MIPs were used, which combined the favorable contrast-to-noise characteristics of source images with the improved overview related to MIPs. Using thin-slab MIPs for the actual identification diminished the disadvantage for Gd-DTPA, as the contrast between collaterals and background was still high enough to identify an equal number of collaterals compared to XRA, leaving little room for improvement with Gadomer. 
One of the main advantages of blood pool agents is their decreased extravasation. For Gadomer, the extravasation rate is approximately 10 times lower compared to Gd-DTPA [114], which has two positive effects for angiography. First, a slower decrease in blood concentration during the distribution phase could be observed, as demonstrated in a previous study [112]. Second, the lower extravasation rate resulted in decreased contrast agent concentrations in the background tissue [114]. To obtain the same vascular contrast enhancement, the dose of Gadomer could be reduced by a factor 2 compared to Gd-DTPA due to a higher $r_{1}$ value for Gadomer.

Although Gadomer has already been safely used for angiography in humans [109], it is not yet approved. The potential advantage of Gadomer over the clinically approved BPA gadofosveset trisodium is that it is remains intravascular due to its intrinsic structure rather than to its binding affinity with albumin. During first-pass, a large (74\% [104]) fraction of gadofosveset trisodium is not yet bound and extravasates more rapidly, as gadofosveset trisodium has a size comparable to Gd-DTPA. Moreover, at 3 Tesla, compared to gadofosveset, Gadomer has a higher $r_{1}$ (Gadomer: $13 \mathrm{~L} \mathrm{mmol}^{-1} \mathrm{~s}^{-1}$; gadofosveset: $9.9 \mathrm{~L} \mathrm{mmol}^{-1} \mathrm{~s}^{-1}$ ) and a lower $r_{2}$ (Gadomer: 25 $\mathrm{L} \mathrm{mmol}^{-1} \mathrm{~s}^{-1}$; gadofosveset: $60 \mathrm{~L} \mathrm{mmol}^{-1} \mathrm{~s}^{-1}$ ), which may favor Gadomer for imaging at 3 Tesla.

\section{MRA versus XRA}

In addition to the advantages regarding invasiveness and ionizing radiation, MRA has the advantage of the availability of 3D data compared to $2 \mathrm{D}$ XRA, which is a one-directional projection. On these projections no distinction can be made between arteries that branch off or cross other arteries, which hinders the identification of stem and re-entry zones. A second disadvantage of XRA is the high signal from bone structures. In clinical practice, this problem is usually solved by using digital subtraction of pre- and post-contrast images. In our study, vasospasms and animal movement due to contrast injection regularly resulted in blurred images which were less suited for collateral identification. Consequently, the proximity of the femur to the mid and re-entry zone of collaterals stemming from the circumflex femoral artery resulted in the identification of fewer collaterals stemming from the CFA on XRA images compared to MRA. Although the spatial resolution achieved with MRA is generally lower than with XRA, MRA compared well to XRA for both contrast agents and the total number of collaterals visualized on MRA was comparable to that of XRA. 


\section{Conclusion}

The blood pool agent Gadomer has great potential in the visualization of small collateral arteries with steady-state contrast-enhanced MRA. The total number of identified collaterals was equal to XRA. Although no difference between Gadomer and Gd-DTPA in number of identified collaterals or interobserver variability was found in this study, the enhanced collateral artery conspicuity appreciably facilitated the identification of collateral arteries with Gadomer compared to Gd-DTPA, while the dose was reduced. 


\subsection{Appendix I: Dose optimization}

\section{Methods}

The optimal dose for steady-state MRA was determined for each contrast agent based on their pharmacokinetic properties in plasma for rabbits, and the computed relation between contrast agent concentration and signal enhancement. The plasma concentration time curve $C_{p}$ in rabbits can be described by a biexponential decay function:

$$
C_{p}(t)=D\left(a_{1} \mathrm{e}^{-t / \tau_{1}}+a_{2} \mathrm{e}^{-t / \tau_{2}}\right)
$$

where $D$ is the administered dose (unit: $\mathrm{mmol} / \mathrm{kg}$ ). Amplitudes $a_{1}$ and $a_{2}$ and time constants $\tau_{1}$ and $\tau_{2}$ were determined by blood sampling in the femoral artery, as described in a previous experiment [112]. Amplitudes $a_{1}$ and $a_{2}$ were found to be 22 and $9.7 \mathrm{~kg} / \mathrm{L}$ for the SCA and 24 and $5.5 \mathrm{~kg} / \mathrm{L}$ for the MCA. $\tau_{1}$ and $\tau_{2}$ were 2.5 and $75 \mathrm{~min}$ for the SCA and 5.5 and $70 \mathrm{~min}$ for the MCA. $C_{\mathrm{p}}$ was converted to the whole blood contrast agent concentration $C_{b}(t)$ by correcting with the hematocrit value $(\mathrm{Hct}=0.40[112])$.

Concentrations were converted into $T_{1}$ and $T_{2}^{*}$ using values from literature for pre-contrast $T_{1}$ and $T_{2}^{*}$ of blood and muscle tissue [115], and $r_{1}$ and $r_{2}^{*}$. $T_{2}$ and $r_{2}$ were used as approximations for $T_{2}^{*}$ and $r_{2}^{*}$. For Gadomer, plasma $r_{1}$ and $r_{2}$ were 13 and $25 \mathrm{~L} \mathrm{mmol}^{-1} \mathrm{~s}^{-1}$ [100] at 3 Tesla, respectively. For Gd-DTPA, $r_{1}$ is 3.7 and $r_{2}$ is $5.3 \mathrm{~L} \mathrm{mmol}^{-1} \mathrm{~s}^{-1}$. Based on these $T_{1}$ and $T_{2}$ values, signal amplifications were calculated using a formula for signal intensity in a spoiled fast gradient echo sequence [116]. Signal enhancement was defined as the ratio between post- and pre-contrast signal intensity.

\section{Results}

Figure 2.3 shows the signal enhancement time course for a range of contrast agent doses for Gadomer (panel a; range: 0.05 - $0.20 \mathrm{mmol} / \mathrm{kg}$ ) and Gd-DTPA (panel b; range $0.10-0.50 \mathrm{mmol} / \mathrm{kg}$ ). The optimal dose for Gadomer was $0.10 \mathrm{mmol} \mathrm{Gd} / \mathrm{kg}$. For Gd-DTPA, maximum signal enhancement was obtained with a dose of $0.50 \mathrm{mmol}$ $\mathrm{Gd} / \mathrm{kg}$, although the difference with doses in the range of $0.2-0.5 \mathrm{mmol} \mathrm{Gd} / \mathrm{kg}$ was small. We therefore selected the lowest dose in this range $(0.20 \mathrm{mmol} / \mathrm{kg})$. 

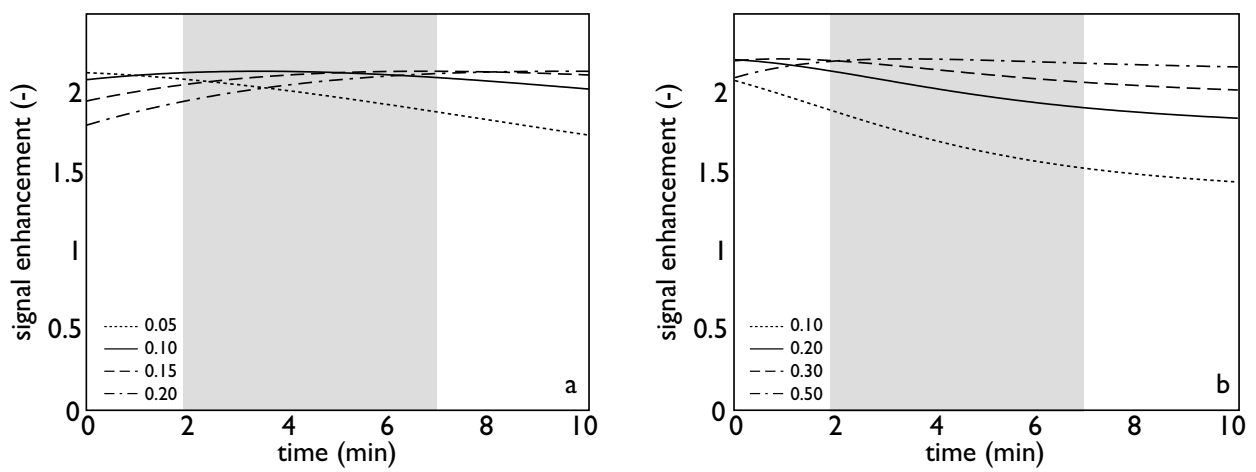

Figure 2.3: Signal enhancement time course for a range of contrast agent doses for Gadomer (panel a; range: $0.05-0.20 \mathrm{mmol} / \mathrm{kg}$ ) and Gd-DTPA (panel b; range $0.10-0.50 \mathrm{mmol} / \mathrm{kg}$ ). Grey shaded regions indicate the acquisition window for steady-state MRA in this study. 

Vessel-enhanced signal intensity distribution analysis for MR angiography of peripheral arteriogenesis 


\section{Abstract}

Purpose: To automatically analyze the time course of collateralization in a rat hind limb ischemia model based on signal intensity distribution (SID).

Materials and Methods: Time-of-flight MR angiograms were acquired in eight rats at 2, 7 and 21 days after unilateral femoral artery ligation. Analysis was performed on maximum intensity projections filtered with vessel enhancement filter. Differences in SID between ligated and control limb were monitored over time and compared to manual collateral artery identification.

Results: The differences in SID correlated well with the number of collateral arteries found with manual quantification. The time courses of ultra-small (diameter « $0.5 \mathrm{~mm}$ ) and small (diameter approximately $0.5 \mathrm{~mm}$ ) collateral artery development could be differentiated, revealing that maturation of the collaterals and enlargement of their feeding arteries occurred mainly after the first week post ligation.

Conclusion: Signal intensity distribution analysis is easy to implement, objective, fast and more sensitive to temporal changes in morphology than manual identification

Provisionally accepted for publication in Journal of Magnetic Resonance Imaging 


\subsection{Introduction}

Collateral arteries developing from pre-existent arterioles can act as natural bypasses in patients with peripheral or coronary arterial occlusive disease [117]. Currently, therapies stimulating the formation of collateral arteries (i.e. arteriogenesis) and restoring the impaired blood supply are explored $[27,94,118]$. Accurate evaluation of such novel treatments strongly depends on image acquisition and analysis techniques that can detect and quantify the vascular responses both in experimental and clinical settings. Previously, non contrast-enhanced inflow MR angiography (MRA) techniques such as time-of-flight (TOF) MRA have been successfully used to non-invasively quantify collateralization in small animal ischemic hind limb models [119-122].

Collaterals are usually identified and quantified manually on (rotating) maximum intensity projections (MIPs) using the Longland definition [60], which requires identification of the collateral stem, mid- and re-entry zone. Visualization of complete trajectories is challenging due to inflow artifacts, flow voids for in-plane arteries [41], and the ultra-small collateral artery calibers $(<0.5 \mathrm{~mm})$. Moreover, manual quantification is time consuming, observer dependent and provides little information on changes in vessel size and physiological impact. A physiologically more relevant measure would be the blood volume or flow of the collateral arteries. Wagner et al. [119] proposed a method to quantify collateral flow based on the dependence between pixel signal intensity and the amount and speed of inflow of unsaturated blood spins. The signal intensity histogram of time-of-flight angiograms may provide surrogate parameters for changes in vessel morphology, as the signal intensity distribution depends on vessel size [123]. A problem to overcome is the small volume fraction of arteries in hind limbs compared to the large volume of background tissue. Projecting the 3D data on a 2D plane using a MIP algorithm increases the fraction of arterial pixels, but simultaneously increases background intensity, which reduces the conspicuity of vessels with small diameters or low signal intensity [124]. Therefore, selective enhancement of vessels using line-enhancing filters such as multi-scale vessel enhancement filters $[125,126]$ is desired to decrease noise and improve signal intensity distribution analysis.

In this study we propose an easy-to-implement automated analysis method, using signal intensity distribution (SID) characteristics to evaluate the time course of arteriogenesis. Time-of-flight MR angiograms were acquired in rats at different time points after unilateral femoral artery ligation. MIPs over the area with collateralization were processed with a 2D vessel enhancement filter. The resulting images were combined and subsequently analyzed with regard to signal intensity distributions. Temporal changes in SID were evaluated and compared with quantification by manual identification of collateral arteries. 


\subsection{Materials and Methods}

\section{Animal model}

This study was approved by the local animal ethics committee. Eight male SpragueDawley rats were included. The right femoral artery was occluded by placing ligations $0.5 \mathrm{~cm}$ below the branch of the circumflex femoral artery and just above the bifurcation of the popliteal and saphenous artery. Anesthesia was induced by placing the animal in an induction box filled with a mixture of oxygen and isoflurane. During both the intervention and MR exams, the rats were ventilated with $3 \%$ isoflurane in oxygen. Analgesia was provided by intramuscular injection of buprenorphine $(0.01$ $\mathrm{mg} / \mathrm{kg}$ ) immediately before the start of surgery.

\section{MR angiography}

On days 2, 7, and 21 post ligation, the animals were imaged in supine position in a 7.0 Tesla MR system with a birdcage quadrature coil (Bruker Biospin, Ettlingen, Germany). The angiography protocol consisted of a multi-slice 2D flow-compensated gradient echo sequence. A flow saturation slab located distally to the imaging plane was applied to suppress venous enhancement. TR/TE were $28 / 4.5 \mathrm{~ms}$ and flip angle was $76^{\circ}$. 150 axial slices with slice thickness $0.55 \mathrm{~mm}$ were acquired. Matrix size was $200 \times 256$ and pixel size was $0.49 \times 0.49 \mathrm{~mm}^{2}$. The number of averages was 2 and total acquisition time was $35 \mathrm{~min}$.

\section{Direction-enhanced signal intensity distribution analysis}

Data processing: For each TOF-MRA data set, maximum intensity projections (MIPs) were computed in the cranio-caudal direction in MATLAB (The MathWorks, Natick, MA). The stack of axial slices over which the MIPs were computed was determined for each set separately based on vascular anatomy. The most proximal slice was chosen just above the branch of the circumflex femoral artery and the most distal slice was positioned above the bifurcation of the cranial and caudal tibial artery (see figure 3.1a).

Filtering: Vessel enhancement was accomplished by applying the 2D multi-scale vessel enhancement filter described by Kagadis et al. [126] to the MIP. This filter is based on eigen analysis of the Hessian matrix $(H)$. $H$ was calculated for each pixel and consisted of the second order Gaussian derivatives of the corresponding input image $I(x, y)$, and was defined as:

$$
H(x, y)=\left(\begin{array}{ll}
I_{x x} & I_{x y} \\
I_{x y} & I_{y y}
\end{array}\right)
$$



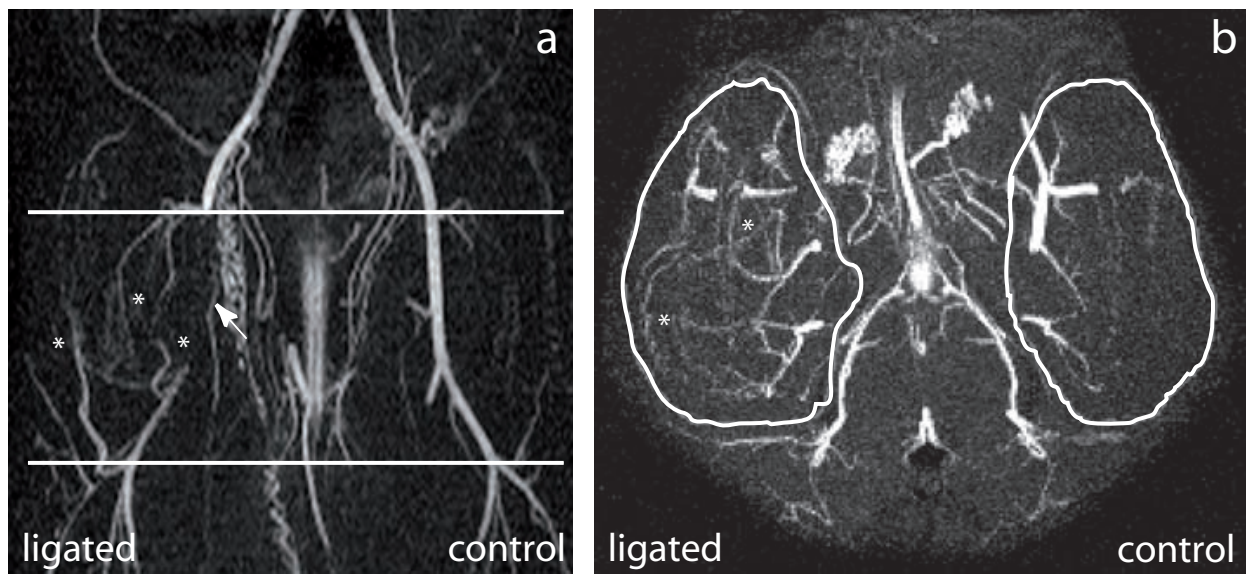

Figure 3.1: Coronal (panel a) and axial (panel b) maximum intensity projections (MIPs) giving an overview of the rat's vascular anatomy on day 21 post ligation. White lines in panel a indicate the slice stack over which the axial MIP (b) is calculated. White lines in panel $b$ indicate the regions of interest in the control and ligated limb. The arrow indicates the ligated part of the femoral artery. Collateral arteries are marked with *. 1: femoral artery; 2 : popliteal artery; 3: saphenous artery; 4: circumflex femoral artery; 5: internal iliac artery; 6: branch of internal iliac artery; 7: superficial caudal epigastric artery; 8: cranial tibial artery; 9: caudal tibial artery.

The Gaussian derivates $I_{\mathrm{xy}}$ of $I(\mathrm{x}, \mathrm{y})$ were calculated by convoluting $I(\mathrm{x}, \mathrm{y})$ with the second order derivates of 2D Gaussian kernels $G(x, y, \sigma)$ with standard deviation $\sigma$ :

$$
I(\mathrm{x}, \mathrm{y}, \sigma)=I(\mathrm{x}, \mathrm{y}) \otimes \frac{\partial^{2}}{\partial \mathrm{x} \partial \mathrm{y}} G(\mathrm{x}, \mathrm{y}, \sigma)=I(\mathrm{x}, \mathrm{y}) \otimes \frac{\partial^{2}}{\partial \mathrm{x} \partial \mathrm{y}} \frac{1}{2 \pi \sigma^{2}} e^{\frac{\mathrm{y}^{2}-\mathrm{x}^{2}}{2 \sigma^{2}}}
$$

Eigen analysis resulted in two eigenvectors and corresponding eigenvalues $\left(\lambda_{1}\right.$ and $\left.\lambda_{2}\right)$. Line-like structures are characterized by $\left|\lambda_{1}\right|>>\left|\lambda_{2}\right|$. By calculating $\lambda_{1}$ for each pixel, resulting in filter $L(x, y)$, vessels with a diameter similar to $\sigma$ were enhanced. As we were mainly interested in enhance ultra-small vessels with a diameter of approximately $0.5 \mathrm{~mm}$ ( 1 pixel), a single $\sigma$ of 0.5 was used. The filtered image was obtained by multiplying $L(\mathrm{x}, \mathrm{y})$ with the original MIP. A schematic overview of the procedure is shown in figure 3.2.

Filter efficacy: The effect of the filter on the enhancement of vessel structures was assessed by determining the contrast-to-noise ratio (CNR) between stationary background tissue and the control femoral artery and a collateral artery at day 21 in the original and filtered MIP. The noise level was estimated by determining the standard deviation of the background tissue.

Signal intensity distribution: In the original MIPs, regions of interest (ROIs, see figure $3.1 \mathrm{~b}$ ) were drawn in both the control and ligated limb. The ROls encom- 


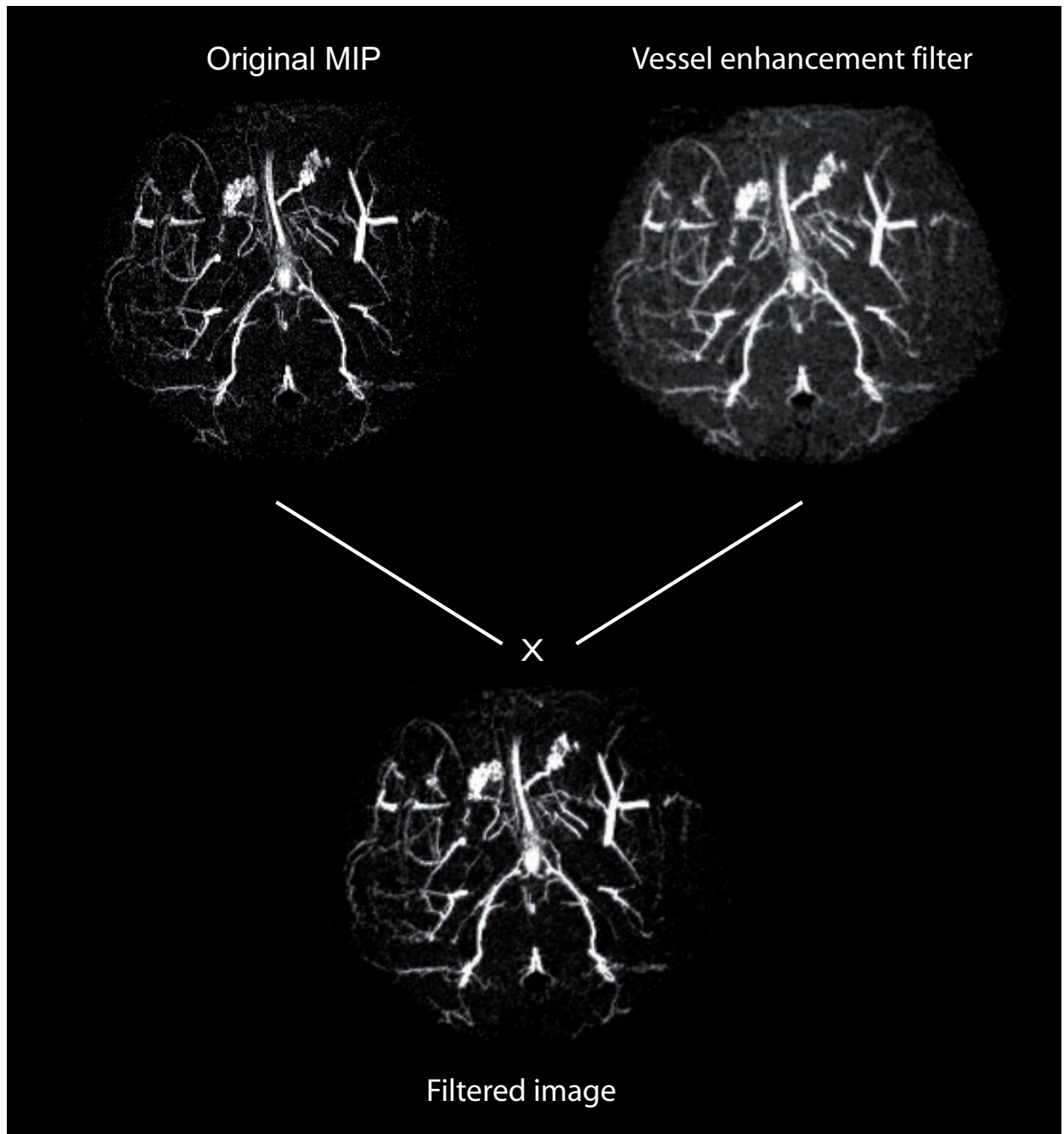

Figure 3.2: Filtering procedure. For each pixel, the Hessian matrix containing the second order partial derivatives of the original maximum intensity projection (MIP) were calculated for a Gaussian kernel with $\sigma=0.5$ pixel. With eigen analysis of the Hessian the largest eigenvalue was calculated for each pixel, resulting in the filter. Finally, the filtered image was obtained by multiplying the original MIP with the filter. 
passed the femoral artery (in the control limb), the circumflex femoral artery and the anterior branches of the internal iliac artery, and excluded the testicular artery, the iliac artery and its posterior branches. Pixel intensities in the ROI were normalized by division with the maximum pixel intensity in the ligated limb. The distribution of pixel intensities was quantified by determining the signal intensities corresponding to various percentile signal intensities $\left(\mathrm{SI}_{x}\right.$, with $x$ the percentage of pixels with signal intensities lower than $\mathrm{SI}_{x}$ ) in the ligated and control limb. Three values for $x$ were defined empirically to reflect ultra-small (diameter «1 pixel or $0.5 \mathrm{~mm}$ ), small (diameter approximately $0.5 \mathrm{~mm}$ ) and large (diameter $>1.5 \mathrm{~mm}$ ). The difference between the ligated and control side was expressed by means of the lateralization index for $\mathrm{SI}_{x}\left(\mathrm{LI}_{x}\right)$ :

$$
L I_{x}=\frac{S I_{x, \text { ligated }}-S I_{x, \text { control }}}{S I_{x, \text { ligated }}-S I_{x, \text { control }}}
$$

$\mathrm{LI}_{x}$ ranges from -1 to +1 and is 0 for equal signal intensities in both $\mathrm{ROls}$. For $\mathrm{LI}_{x}$ $>0$, the $x$ percentile signal intensity is higher in the ligated limb, and for $L_{x}<0$ the control limb has the higher $\mathrm{SI}_{x}$. $\mathrm{LI}_{x}$ was hypothesized to represent collateralizationrelated differences in the number of arteries of various calibers.

\section{Collateral quantification}

The number of visible collateral arteries was counted by two independent observers in the medical image software application OsiriX (version 3.7), using axial maximum intensity projections in the cranio-caudal direction of the original, unfiltered data over a limited range of axial slices. The thickness and location of the slab could be adjusted in real time to obtain optimal depiction of the collaterals. Collateral identification was preferably based on the Longland definition 9, which requires identification of the stem, mid- and re-entry zone. However, vessels with a clear mid-zone and either a stem or re-entry zone were also considered to be collateral arteries.

\section{Statistics}

Inter-observer agreement for collateral artery counting was assessed with a betweenobserver coefficient of variation ( $\mathrm{CoV}$ ), which is defined as the average standard deviation between the two observers divided by the average number of collateral arteries. The differences in $\mathrm{LI}_{x}$, and number of collaterals between days 2, 7 and 21 were tested using a paired Student's $t$-test. The correlation between $\mathrm{LI}_{x}$ and number of collaterals in individual rats was determined using a Pearson correlation coefficient. Effects were considered significant for $p<0.05$. 

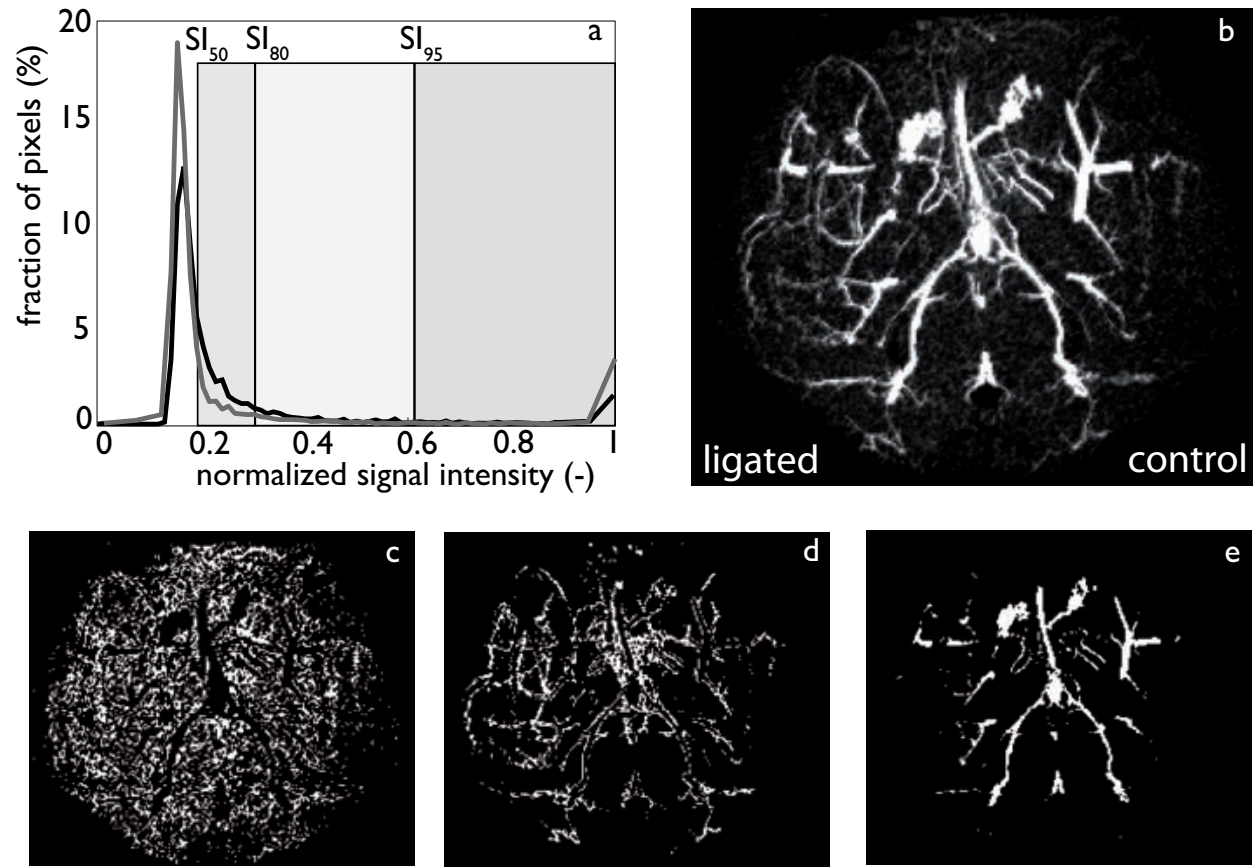

Figure 3.3: Histogram of signal intensities in the ligated (black line) and control limb (grey line), including the 50, 80 and $95 \%$ percentile signal intensities ( $\mathrm{SI}_{50}, \mathrm{SI}_{80}$ and $\mathrm{SI}_{95}$, respectively) for the ligated limb. Panel $b$ shows the corresponding filtered maximum intensity projection. Panels $\mathrm{c}, \mathrm{d}$ and e show the segmentation according to pixel signal intensity. Panel c shows pixels with signal intensities between $\mathrm{SI}_{50}$ and $\mathrm{SI}_{80}$. Note that the ligated side contains more pixels in this category. Panel $d$ represents pixels with signal intensities between $\mathrm{Sl}_{80}$ and $\mathrm{SI}_{95}$. In the ligated limb, line-like structures can be noted, which correspond to the visible collateral arteries in panel b. Panel e shows pixels with $\mathrm{SI}>\mathrm{Sl}_{95}$. In the control limb, the femoral artery and its main branches can be distinguished. In the ligated limb the femoral artery was absent, but the branches were large compared to the control side. The image was acquired on day 21 . 


\subsection{Results}

\section{Filter efficacy}

The vessel enhancement filter significantly increased the CNR in the femoral artery by $40 \%\left(\mathrm{CNR}_{\text {unfiltered }}=40 \pm 7(\right.$ mean $\pm \mathrm{SD})$ and $\left.\mathrm{CNR}_{\text {filtered }}=56 \pm 9 ; p<0.01\right)$. In the collateral arteries, CNR increased by a $100 \%\left(\mathrm{CNR}_{\text {unfiltered }}=10 \pm 1\right.$ and $\left.\mathrm{CNR}_{\text {filtered }}=21 \pm 4 ; p<0.01\right)$.

\section{Vessel-enhanced signal intensity distribution analysis}

Figure 3.3 shows the segmentation according to pixel intensity. Pixels with intensities above $\mathrm{Sl}_{95}$ (as measured in the ROI in the ligated limb) corresponded to large arterial structures such as the femoral artery and its main branches. These structures were surrounded by pixels with intensities between $\mathrm{SI}_{80}$ and $\mathrm{SI}_{95}$, which also were part of individual, small line-like structures in the ligated limb. The number of pixels with signal intensity between $\mathrm{SI}_{50}$ and $\mathrm{SI}_{80}$ was higher in the ligated limb compared to the control limb. Further analysis of lateralization indices $\mathrm{LI}_{x}$ was performed with $x$-values of 50,80 and $95 \%$.

Compared to the control limb, the main signal intensity peak in histogram was shifted towards higher signal intensities (see figure 3.4). This shift increased over time, and coincided with an increased number of visible line-like structures on the filtered MIP. In the control limb, a larger fraction of pixels with normalized signal intensities higher than 0.95 was found compared to the ligated limb. This difference decreased over time.

The lateralization indices $\mathrm{LI}_{50}, \mathrm{LI}_{80}$ and $\mathrm{L} \mathrm{I}_{95}$ were significantly different from 0 at all time points and increased over time (figure 3.5). L $\mathrm{I}_{80}$ was larger than 0 (i.e. $\mathrm{SI}_{80}$ was higher in the ligated limb compared to the control limb) and became significantly higher at day 21 compared to days 2 and $7(p=0.02)$. LI 50 followed the same trend (day 2 versus day 21: $p=0.10$; day 7 versus day 21: $p=0.13$ ). Ll l95 $_{5}$ was negative and increased over time: Ll 95 was significantly higher on day 21 compared to both day 2 and day 7 ( $p<0.01$ for both). The difference in LI between days 2 and 7 was not significant for any of the SI-values.

\section{Comparison with manual collateral artery quantification}

The number of collateral arteries identified manually, averaged for the two observers, displayed similar trends compared to $\mathrm{LI}_{x}$, increasing from $7.3 \pm 0.9$ (mean \pm standard error) on day 2 to $11.4 \pm 0.5$ on day 21 (see figure $3.5 \mathrm{~d}$ ). While for the SID analysis the difference between day 7 and 21 was significant, the number of counted collaterals 

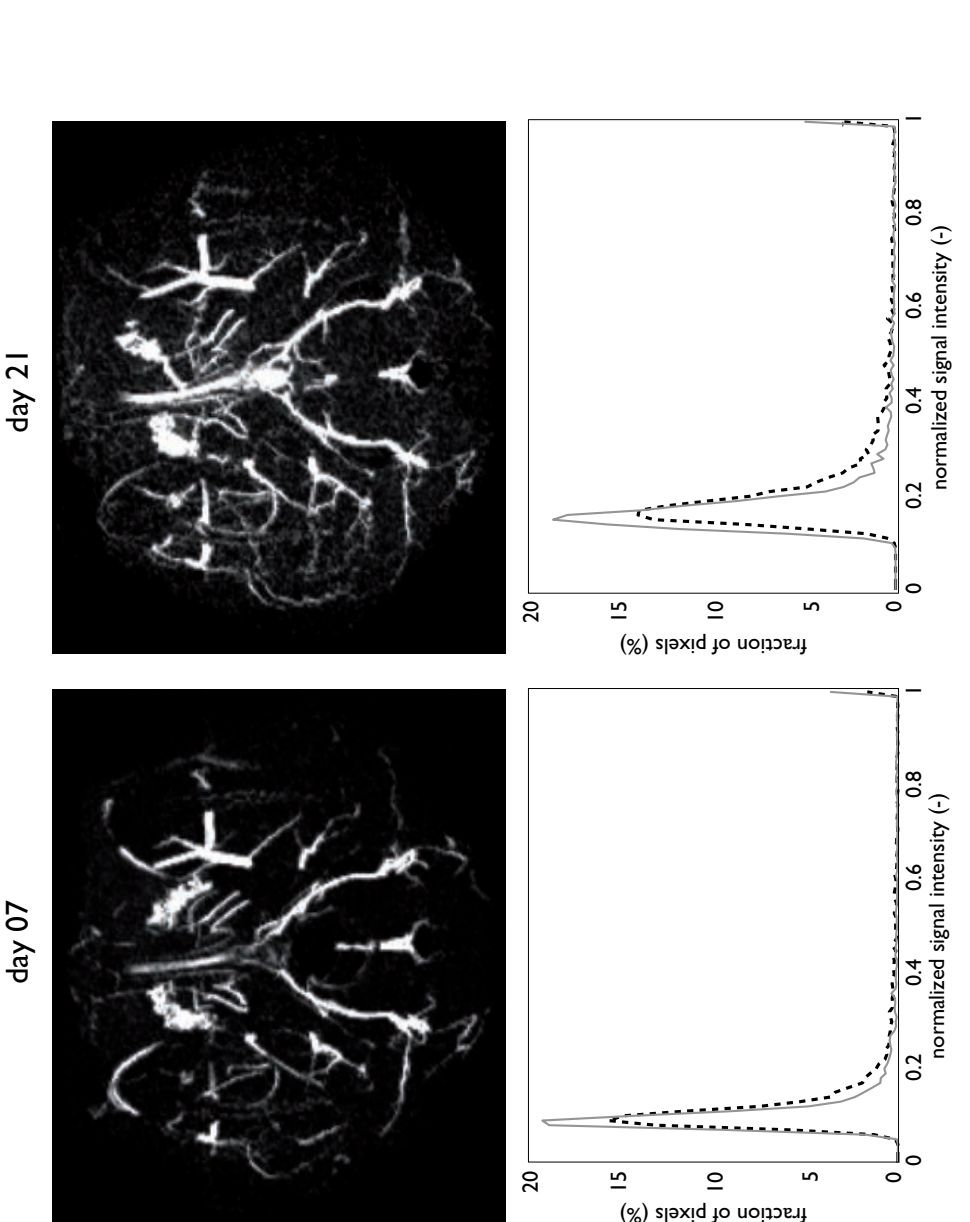

그 $\frac{\pi}{\sqrt{0}}$

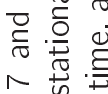

i $\stackrel{0}{\infty} \stackrel{0}{0}$

ते

$\pi$

३)

党空

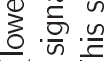

o $3 \vdash$

结

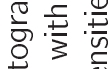

苞 $\frac{n}{0} \stackrel{0}{0}$

잉 $\stackrel{x}{\circ}$

흗ำ

की

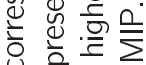

u d $\frac{0}{0}$

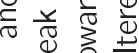

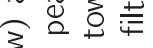

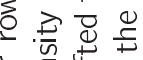

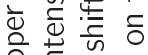

을. ज记

जิ $\stackrel{0}{5}$

高䨌

$\sum \stackrel{5}{5}$

윰
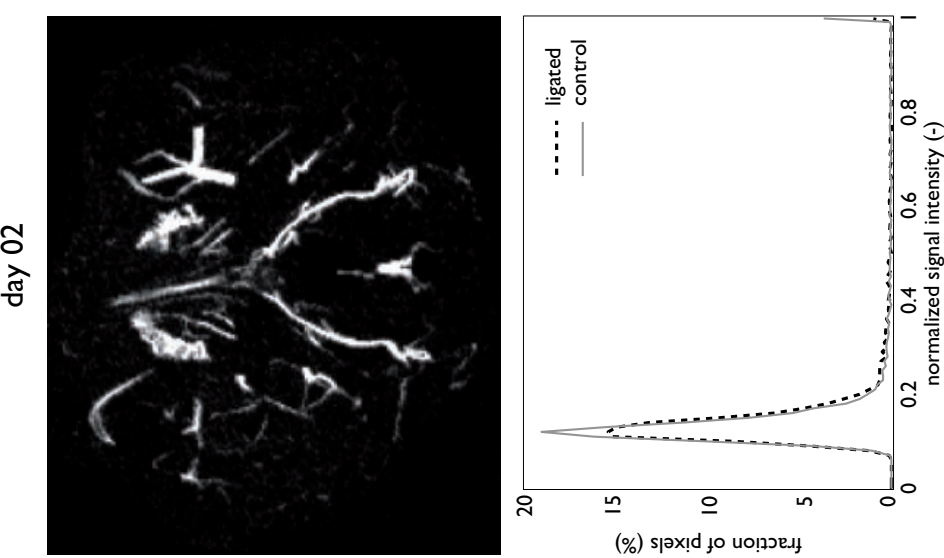

ह

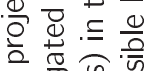

믐에

专曹

¿

.

छ ह

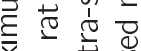

离

ป

ठ․ $\cong$

๖․ำ 든

它泀罂

$\ddot{\gamma} \bar{z}$

mi 苍

늠 흔

ป 는

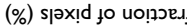



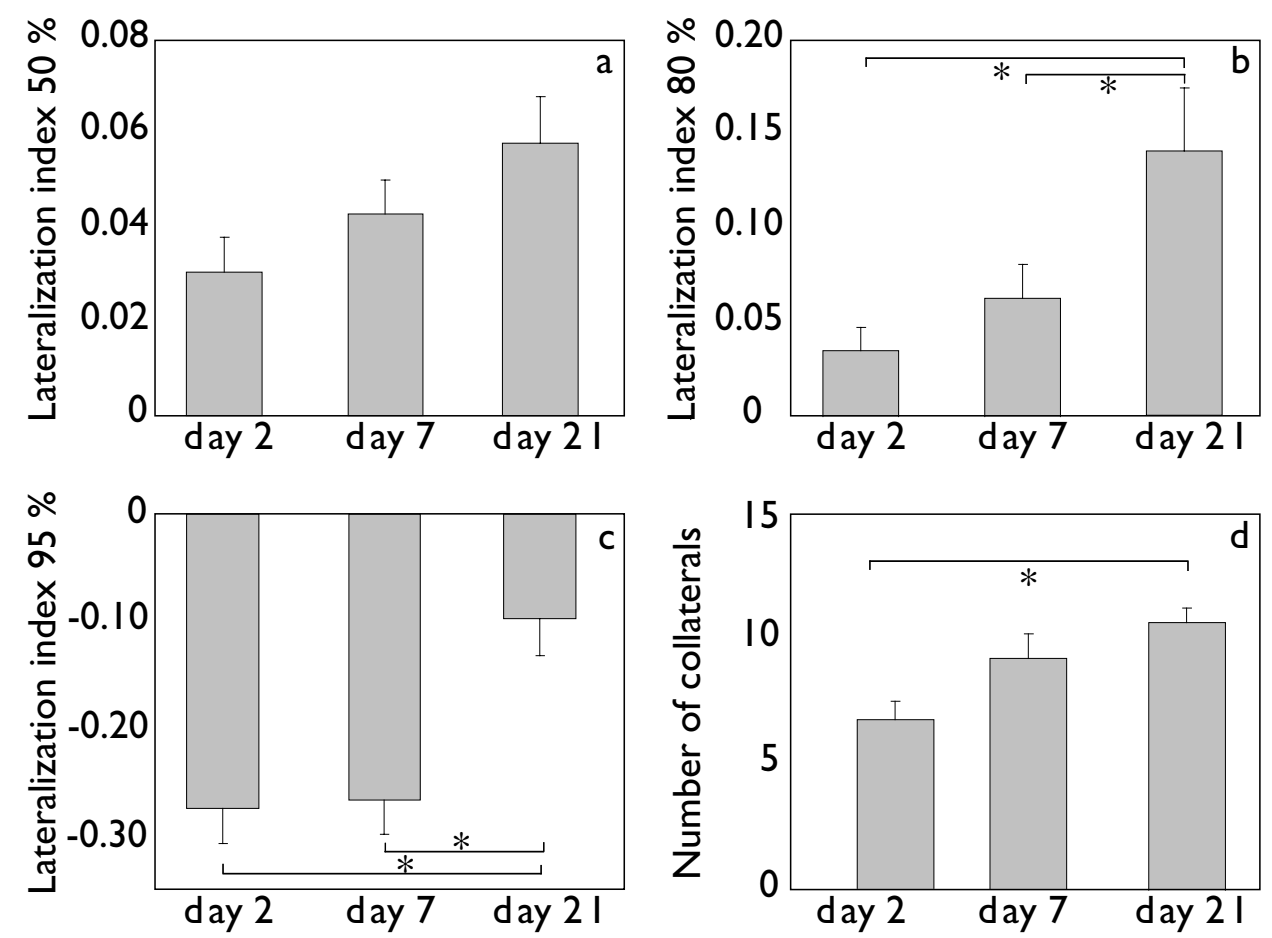

Figure 3.5: Lateralization index for 50, 80 and $95 \%$ percentile signal intensity values (panels a, b and c respectively) and number of collateral arteries (panel d) at days 2,7 and 21 post ligation. Significant differences in LI between days $(p<0.05)$ are indicated with *.

was only significantly higher on day 21 compared to day $2(p<0.01)$. Inter-observer variation in the number of identified collaterals was $19 \%$ on day 2 and $14 \%$ on days 7 and 21 , meaning that the difference between observers was approximately $1-2$ collateral arteries.

A significant correlation was found between the number of identified collateral arteries and all lateralization indices. The correlation was strongest with $\mathrm{Ll}_{80}$ (Spearman correlation coefficient $R=0.67(p<0.01)$, compared to $R=0.52(p=0.01)$ with $\mathrm{LI}_{50}$ and $R=0.56$ with $\mathrm{LI}_{95}(p<0.01)$.

Compared to manual identification, the automated method was considerably faster. Manual identification took approximately ten minutes per animal, whereas for signal intensity distribution analysis two minutes were needed, consisting of one minute of automated computer calculation time and one minute of user input for determining the location of the MIP slab in the original data and drawing the ROIs. 


\subsection{Discussion}

In this study, an MRI-based image analysis strategy was proposed for the automated assessment of collateral artery development in a hind limb ischemia model. From TOF-MR angiograms, axial MIPs encompassing the newly developed collateral arteries were generated. The conspicuity of collateral arteries on these MIPs was strongly enhanced with a vessel enhancement filter. Signal intensity distributions (SID) characteristics such as the 50, 80 and $95 \%$ percentile intensities were compared to the number of collaterals identified on the source images by two independent observers. Both the number of identified collaterals and differences in SID between control and ligated limb, expressed in lateralization indices (LI), increased over time. LIs correlated well with the number of manually counted collateral arteries.

We looked at three characteristic quantiles in the signal intensity histogram: the 50, 80 and $95 \%$ percentiles. In the histogram, the contribution of ultra-small collateral arteries with signal intensities somewhat higher than the mean background signal cannot be separated from the main bulk of noisy background signal. However, a shift in the median signal intensity (or $\mathrm{SI}_{50}$ ) in the ligated limb compared to the control limb, i.e. a positive lateralization index $\left(\mathrm{LI}_{50}\right)$, indicated that more pixels representing ultra-small arteries with sub-voxel diameters were present.

Pixels with signal intensities higher than $\mathrm{Sl}_{95}$ were found to be specific for large arterial structures such as the femoral artery and its main branches. In the ligated limb, $\mathrm{SI}_{95}$ was lower compared to the control side. The absence of flow in the ligated part of the femoral artery removed the main source of high intensity pixels. Over time, the branches feeding the collateral arteries visibly increased in diameter, resulting in a decreasing difference between the ligated and control side. Changes in $\mathrm{Ll}_{95}$ therefore provide information on the arterial branches feeding the collaterals. Signal intensities between $\mathrm{SI}_{80}$ and $\mathrm{SI}_{95}$ were found to form line-like structures coinciding with visible collateral arteries in the MIP. These vessels are still very small (diameter approximately $0.5 \mathrm{~mm}$ ) compared to the femoral artery. Since flow increases rapidly for increasing diameter (flow $\sim$ diameter $^{4}$, assuming laminar flow), they contribute substantially more to flow recovery than the ultra-small vessels [97].

Signal intensity distribution analysis produces surrogate measures for changes in vasculature. The degree to which the various lateralization indices are true representative for physiological properties such as arterial volume and flow is hard to validate in the absence of a good gold standard. Invasive catheter x-ray angiography has been used to validate collateral artery quantification, showing a good correlation with the number of vessels counted on MR angiograms [97], but it does not give accurate information on vessel size and volume. By showing the segmentation of the MIP based on signal intensity ranges we have sought to demonstrate that the various lateralization indices correspond to the presence of arterial structures of varying size. Moreover, correlation analysis with the number of manually identified collateral arteries showed that the parameters are closely related. This makes SID analysis a 
good alternative for manual counting, with the benefit of more detailed information about arterial structures of various sizes.

Both signal intensity distribution analysis and manual identification revealed that collateral arteries had already started to develop as early as day 2 post ligation. The number of vessels counted manually grew steadily over time, but only the difference between day 2 and 21 was statistically significant. The SID method seemed more sensitive to changes in vasculature, as it could differentiate the time courses of small and ultra-small collateral artery development. It showed that there was a significant change regarding collateral arteries with diameters of approximately $0.5 \mathrm{~mm}$ between days 7 and 21 . Moreover, during this period the arterial branches feeding these vessels increased in volume. Both processes indicated that maturation of the collaterals mainly took place during this period.

We chose to use a filter based on a multi-scale vessel enhancement filter to increase the contrast between vessels and background. This type of filter is frequently used in medical image analysis $[125,127]$. Filtering is commonly used as a preprocessing step before segmenting the vascular tree from the background. Many segmenting strategies are available (for an overview, see [128]), including histogram-based techniques for TOF-MRA [129]. However, segmentation of vessels with a diameter < 5 pixels is considered nearly impossible based on overlapping signal intensity distributions with background tissue $[123,130]$. Even though segmentation of collateral arteries may not be achievable, filtering was beneficial for signal intensity distribution analysis. For the signal analysis distribution to work, it is necessary to separate the signal intensities of vascular structures from the noisy background as much as possible. Filtering suppressed the noise and improved the CNR of small collateral by a factor 2, making the SID approach more sensitive.

In conclusion, with signal intensity distribution analysis and vessel enhancement filtering of time-of-flight MR angiograms, the time course of peripheral arteriogenesis can be monitored. Lateralization indices for 50, 80 and 95 percentile signal intensities provide information on ultra-small ( $« 0.5 \mathrm{~mm}$ ), small (approximately $0.5 \mathrm{~mm}$ ) and larger arterial structures $(>1.5 \mathrm{~mm})$, respectively. All three measures correlated well with the number of collateral arteries found by manual identification. The method is easy to implement, objective, fast and more sensitive to temporal changes than manual identification. 



\section{Reliability of pharmacokinetic parameters: small versus medium-sized contrast agents}




\section{Abstract}

Current clinical applications of dynamic contrast-enhanced magnetic resonance imaging are based on the extravasation of relatively small contrast agents (SCAs). SCAs are considered disadvantageous, as they require high image sampling rates. Mediumsized contrast agents (MCAs) leak more slowly into tissue and allow longer dynamic acquisition times, enabling improved image quality. The influence of molecular size on the reliability of pharmacokinetic parameters, including the transfer constant $K^{\text {trans }}$, was investigated.

Computer simulations were performed, with in vivo measured arterial input functions, to determine the bias and variance of pharmacokinetic parameters as a function of contrast agent size, sampling frequency, noise level, and acquisition time.

Better reliability of all parameters was obtained for the MCA compared to the SCA. To obtain similar variance $(10 \%)$ in $K^{\text {trans }}$, the sampling frequency for the SCA $\left(28 \mathrm{~min}^{-1}\right)$ had to be 20 times faster than for the MCA $\left(1.3 \mathrm{~min}^{-1}\right)$. Optimal reliability in parameter estimation required longer acquisition times for MCAs (13 min for $v_{e}$ and 5 min for $\left.K^{\text {trans }}\right)$ than for SCAs $\left(1.7 \mathrm{~min}\right.$ for $K^{\text {trans }}$ and $\left.v_{e}\right)$. Reliable estimation of $v_{p}$ was only achieved with MCAs.

In conclusion, MCAs provided superior reliability for pharmacokinetic parameter estimation compared to SCAs.

Published in Magnetic Resonance in Medicine 2009 


\subsection{Introduction}

Dynamic contrast-enhanced magnetic resonance imaging (DCE-MRI) is a widely applied non-invasive in vivo technique to evaluate tissue microvasculature. It is an established diagnostic method in oncology [68-70]. Additionally, it can be used for monitoring anti-cancer therapy [71-74] or revascularization and recovery of ischemia in skeletal muscles [75-78]. During DCE-MRI measurements, dynamic images are acquired to measure the tissue wash-in and wash-out of a contrast agent. Subsequently, quantitative pharmacokinetic modeling can be applied to obtain information about microvascular properties, including tissue perfusion, vessel permeability and vascular volume fraction.

The most commonly applied contrast agents are small contrast agents (SCAs), e.g. gadopentetate dimeglumine (Gd-DTPA, molecular weight $0.5 \mathrm{kDa}$ ), as they are rapidly cleared from the body and are approved for human application. The extravasation of SCAs is relatively fast and increases with the microvascular flow (flow-limited) in both normal and hyperpermeable tissue. The rapid extravasation is considered a disadvantage, as it requires fast imaging protocols [131] at the expense of image quality. For the differentiation between normally and abnormally perfused tissue, as well as monitoring of microvascular changes over time, it is of major importance to have a method that provides the highest possible reliability in parameter estimation. In the last ten years extensive research has been performed that improved pharmacokinetic modeling [132-136]. However, DCE-MRI would also benefit from the use of larger contrast agents, which have reduced leakage rates [90]. Such contrast agents would allow longer dynamic acquisition times, which could be employed to improve image quality by increased signal-to-noise ratio, spatial resolution, and/or spatial coverage.

An interesting group of contrast agents is the so called 'rapid clearance blood pool agents' [137]. They are larger than SCAs but small compared to typical macromolecular contrast agents, e.g. albumin-(Gd-DTPA) 30 (molecular weight: 92 kDa) and can therefore be classified as medium-sized contrast agents (MCAs) [74, 138]. They combine the advantage of clearance by glomerular filtration with a lower leakage rate, which is to a large extent determined by the permeability of the vessel wall (permeability-limited). This group includes P792 (gadomelitol, Guerbet, Roissy, France; molecular weight $6.47 \mathrm{kDa}$ ) and Gadomer (Bayer Schering Pharma AG, Berlin, Germany). Gadomer has a molecular weight of $17 \mathrm{kDa}$, but as a result of its globular shape, the apparent molecular weight is $32 \mathrm{kDa}$ [108]. Its potential value in experimental oncology was already demonstrated for the differentiation of malignant and benign tumors [91-93] and the monitoring chemotherapy [73] or antiangiogenesis therapy [74]. Reperfusion injury [139] and hyperpermeable angiogenic tissue could be detected in skeletal muscles, and white muscles could be differentiated from more strongly vascularized red muscles [77].

Monte Carlo simulations may give valuable insight in the uncertainty of pharma- 
cokinetic parameter estimation and enable comparison of various pharmacokinetic models $[140,141]$, contrast administration protocols [87], or measurements settings avoiding extensive in vivo experiments. The influence of the sampling frequency on the reliability of pharmacokinetic parameters was already investigated in various publications $[88,89,142]$ and provided insight in the minimally required sampling frequency. Previously, it was demonstrated that the reliability of pharmacokinetic parameters depends on the magnitude of $K^{\text {trans }}[77,87,89]$. Therefore, imaging protocols that were optimized for DCE-MRI using SCAs in leaky tumor tissue (relatively high $K^{\text {trans }}$ ) are unlikely to be optimal for MCAs in muscle tissue (relatively low $K^{\text {trans }}$ ), which means that optimal experimental settings for MCAs have to be re-defined.

In this study, the reliability of pharmacokinetic parameter estimation and the influence of measurement settings were investigated for an SCA and an MCA. To this extent, the mathematical structure of the extended Kety-model [82] was analyzed and the implications of discrete sampling on $K^{\text {trans }}$ estimation were investigated in both the frequency and time domain. Subsequently, Monte Carlo simulations were performed to investigate the influence of sampling frequency, acquisition time, and signal-to-noise ratio (SNR) on the reliability of pharmacokinetic parameters in terms of bias and variance. 


\subsection{Materials and Methods}

\section{Pharmacokinetic model}

The generalized pharmacokinetic model without a vascular fraction [83] describes the transfer of a contrast agent from the blood plasma into the interstitial space by

$$
v_{\mathrm{e}} \frac{\mathrm{d} C_{\mathrm{e}}}{\mathrm{d} t}=K^{\mathrm{trans}}\left(C_{\mathrm{p}}-C_{\mathrm{e}}\right)
$$

where $C_{p}$ and $C_{e}$ are time-dependent functions that describe the concentrations of the contrast agent in the blood plasma and extravascular extracellular space, respectively. Frequently, $C_{p}$ is approximated by a so-called arterial input function (AIF), which is the concentration time course determined in a large artery. The parameter $v_{e}$ represents the fraction of the extravascular extracellular space into which the contrast agent distributes. The transfer constant $K^{\text {trans }}$ describes the initial leakage rate as it follows from eq. 4.1 by

$$
K^{\text {trans }}=\left.v_{e} \frac{\mathrm{d} C_{e} / \mathrm{d} t}{C_{\mathrm{p}}}\right|_{t=0^{+}}
$$

where $t=0^{+}$represents the time-point just after contrast arrival. The solution of the differential eq. 4.1 is

$$
C_{e}(\mathrm{t})=\frac{K^{\mathrm{trans}}}{v_{\mathrm{e}}} \int_{0}^{t} C_{\mathrm{p}}(\mathrm{u}) \mathrm{e}^{-\frac{K^{\mathrm{trans}}}{v_{\mathrm{e}}}(t-\mathrm{u})} \mathrm{du}
$$

For an MCA, where most of the contrast agent remains in the vascular space, the fractional blood plasma volume $v_{p}$ should not be neglected [82]. After inclusion of $v_{p}$, the tissue concentration $C_{t}$ is composed of the blood plasma and the extravascular extracellular contribution:

$$
C_{\mathrm{t}}(t)=v_{\mathrm{p}} C_{\mathrm{p}}(\mathrm{t})+K^{\mathrm{trans}} \int_{0}^{t} C_{\mathrm{p}}(\mathrm{u}) \mathrm{e}^{-\frac{K^{\mathrm{trans}}}{v_{\mathrm{e}}}(t-\mathrm{u})} \mathrm{du}
$$

Using pharmacokinetic modeling the physiological parameters $K^{\text {trans }}, v_{\mathrm{e}}$, and $v_{\mathrm{p}}$ can be estimated from the time courses $C_{\mathrm{p}}$ and $C_{\mathrm{t}}$ derived from the dynamic measurement. Note that in theory only $K^{\text {trans }}$ depends on the molecular weight, where $K^{\text {trans }}$ decreases for increasing molecular weight [74].

Another way to represent the model is to describe the relationship between $C_{p}$ and $C_{t}$ in the frequency domain. The rationale for using the frequency domain description is that the tissue transfer model gives valuable insights in the effects of sampling frequency, the pharmacokinetic parameters and how low and high frequency components of the AIF are reflected in the tissue enhancement curves. Applying the 

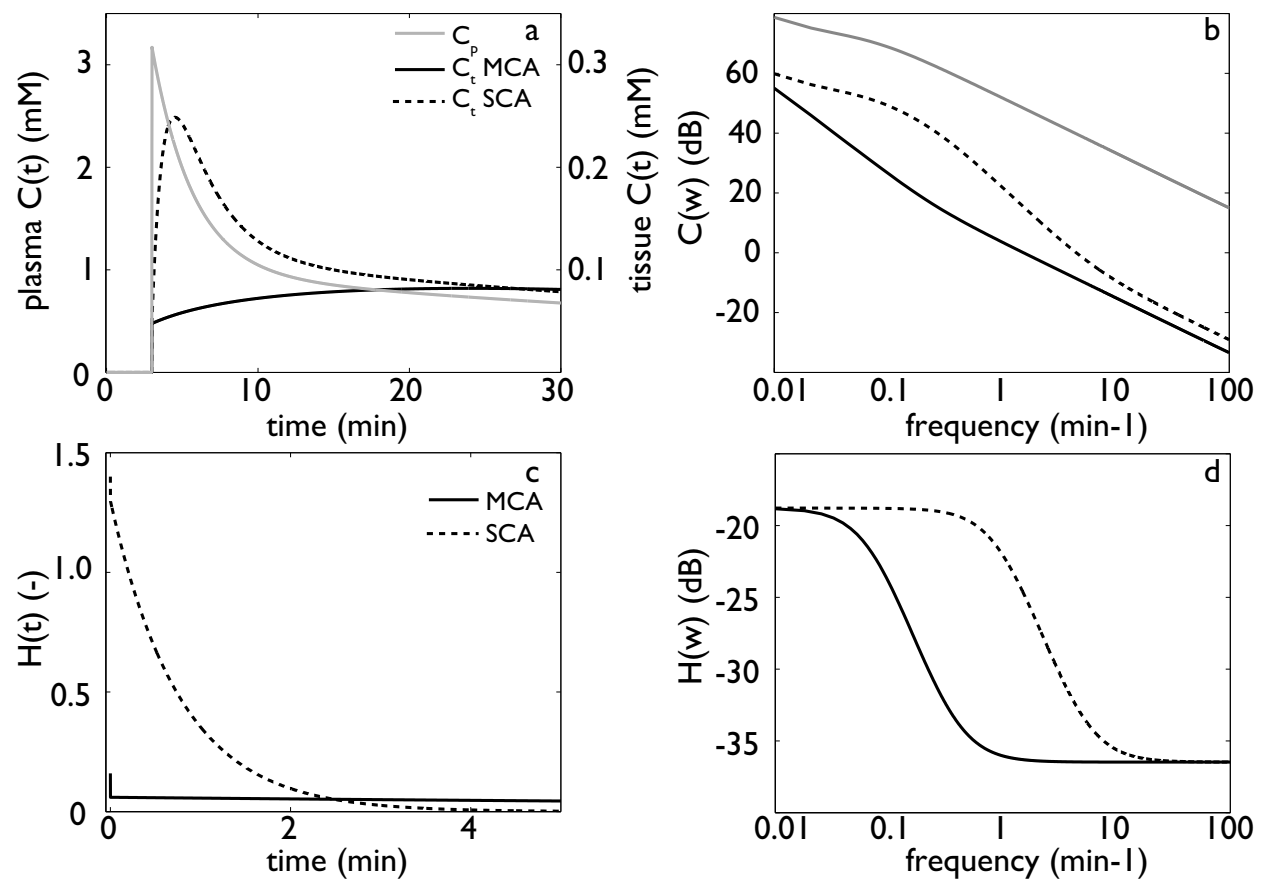

Figure 4.1: Plasma $\left(C_{p}\right)$ and tissue $\left(C_{t}\right)$ concentration curves in the time (a) and frequency domain (b) for both a medium-sized contrast agent (MCA) with $K^{\text {trans }}=0.006 \mathrm{~min}^{-1}$, and a small contrast agent (SCA) with $K^{\text {trans }}=0.13 \mathrm{~min}^{-1}$. The corresponding impulse response functions $H(\mathrm{t})$ and transfer function $H(\omega)$ (panels $\mathrm{c}$ and d, respectively) are also shown. Gray solid lines represent $C_{p}$; black dashed lines represent the SCA and black solid lines the MCA tissue concentration curves. Note the higher frequency content of the SCA curve compared with the MCA.

convolution theorem to eq. 4.4 , the model can be rewritten in the frequency domain as:

$$
C_{t}(\omega)=H(\omega) \cdot C_{p}(\omega)
$$

where $\omega$ is the angular frequency in rad $\mathrm{s}^{-1}, C_{\mathrm{p}}(\omega)$ and $C_{\mathrm{t}}(\omega)$ are the Fourier transforms of $C_{p}(t)$ and $C_{t}(t)$, respectively. Figures $4.1 \mathrm{a}$ and $\mathrm{b}$ illustrate examples of $C_{\mathrm{p}}$ and $C_{t}$ for two contrast agents with different $K^{\text {trans }}$ values, based on the same $C_{p}$ curve, in both the time and frequency domain. The tissue transfer function $H$ in the time and frequency domain is shown in figure 4.1c and d, respectively. $H(\omega)$ is given by [89]:

$$
H(\omega)=v_{\mathrm{p}}+\frac{K^{\text {trans }}}{i \omega+K^{\text {trans }} / v_{\mathrm{e}}}
$$


Essentially, $H(\omega)$ is a low-frequency pass filter with a fraction of the tissue signal $C_{t}$ that is proportional to the input function $C_{p}$. Generally, $H(\omega)$ is expressed in decibel $(\mathrm{dB})$, by transformation to the modulus function $M(\omega)=20 \cdot{ }^{10} \log |H|$. The three pharmacokinetic parameters represent characteristic features in the frequency diagram of $H$. At low frequencies $H(\omega \rightarrow 0)=v_{e}+v_{p}$, representing the maximal distribution volume fraction of the contrast agent. At very high frequencies $H(\omega \rightarrow$ $\infty)=v_{p}$, which represents the vascular distribution volume of the contrast agent. $H(\omega)$ has two characteristic frequencies. The pole of $H(\omega)$ at $f_{\mathrm{p}}=\frac{K^{\text {trans }}}{2 \pi v_{\mathrm{e}}}$ determines the cut-off frequency, i.e. the point at which $M(\omega)$ is attenuated by $3 \mathrm{~dB}$ relative to $M(0)$. The zero frequency of $H(\omega)$, at which $M(\omega)$ is $3 \mathrm{~dB}$ higher than the proportion of the vascular component is at $f_{z}=\frac{K^{\text {trans }}}{2 \pi}\left(\frac{1}{v_{\mathrm{e}}}+\frac{1}{v_{\mathrm{p}}}\right)$.

The Nyquist sampling theorem states that the maximal frequency $f_{\max }$ in the sampled signal equals $f_{\max }=\frac{1}{2} f_{\mathrm{s}}$, where $f_{\mathrm{s}}$ is the sampling frequency. The minimal (non-zero) discrete frequency $f_{\min }$ represented in $f_{\mathrm{s}}$ is defined by $f_{\min }=\frac{1}{T_{\text {aca }}}$, with $T_{\text {acq }}$ the total acquisition time. To obtain reliable pharmacokinetic parameters, all frequencies that contain information about these parameters need to be acquired. The high frequency plateau in $H(\omega)$ contains information on $v_{p}$, so for reliable estimation $f_{\mathrm{s}}$ needs to be at least $2 \times f_{\mathrm{z}}$, the zero frequency that marks the transition to the high frequency plateau. Reliable estimation of $K^{\text {trans }}$ requires that both $f_{\mathrm{p}}$ and $f_{\mathrm{z}}$ are included. The low frequency plateau depends on $v_{e}$, so for good estimation the lowest frequency needs to be smaller than $f_{\mathrm{p}}$.

As $K^{\text {trans }}$ decreases with increasing molecular size, $f_{\mathrm{p}}$ and $f_{\mathrm{z}}$ decrease proportionally and $H(\omega)$ shifts towards the lower frequencies. This will affect the requirements on sampling frequency and acquisition time. Knowledge of the approximate temporal behavior of the contrast agent can therefore be used to optimize the acquisition set-up.

\section{Contrast agents}

Two different $T_{1}$-shortening MRI contrast agents were used: Gd-DTPA (Magnevist, Bayer Schering Pharma AG, Berlin, Germany) and Gadomer, representing an SCA and an MCA, respectively. Table 4.1 provides an overview of the physicochemical properties of the contrast agents.

\section{Arterial input function}

Experiments were approved by the local animal ethics committee. To determine the time course of plasma contrast agent concentration, arterial blood was sampled from three New Zealand White rabbits (weight: $3.0-3.1 \mathrm{~kg}$ ). During the procedure the rabbits were ventilated with $2-3 \%$ isoflurane in oxygen. A canule was placed in the right femoral artery and 24 samples of $0.5 \mathrm{~mL}$ blood were collected at increasing 
Table 4.1: Physicochemical properties of Gd-DTPA and Gadomer

\begin{tabular}{|c|c|c|}
\hline & Gd-DTPA & Gadomer \\
\hline Vial concentration $(\mathrm{mol} \mathrm{L}-1)$ & 0.5 & 0.5 \\
\hline$T_{1}$ relaxivity at $3 T$ in plasma $\left(L \mathrm{mmol}^{-1} \mathrm{~s}^{-1}\right)[100]$ & 3.7 & 13 \\
\hline Molecular weight (kDa) & 0.5 & 17 \\
\hline Number of $\mathrm{Gd}^{3+}$ ions per molecule & 1 & 24 \\
\hline Dose (mmol/kg body weight) & 0.15 & 0.1 \\
\hline
\end{tabular}

time intervals after injection of $0.15 \mathrm{mmol} / \mathrm{kg}$ Gd-DTPA or $0.1 \mathrm{mmol} / \mathrm{kg}$ Gadomer at $0.05 \mathrm{~mL} / \mathrm{s}$ in the ear vein. Prior to injection, two blood samples were taken for the determination of the intrinsic $T_{1}$ relaxation rate of blood $\left(R_{10}\right)$. Each animal received both contrast agents, with a one week interval.

$T_{1}$ relaxation rate $\left(R_{1}\right)$ measurements of the blood samples were performed to determine the contrast agent concentrations. Measurements were performed on a 3 Tesla MRI system (Philips Achieva, Philips Medical Systems, Best, The Netherlands) equipped with a quadrature transmit/receive head coil. Inversion recovery image series were acquired with varying inversion time (TI) 50, 75, 100, 250, 500, 750, 1000, 1500, and $2000 \mathrm{~ms}$, TR $3000 \mathrm{~ms}$, TE $9.0 \mathrm{~ms}$, flip angle 90 ${ }^{\circ}$, and 2 signal averages. Acquired voxel size was $0.7 \times 0.7 \times 3.0 \mathrm{~mm}^{3}$.

The inversion recovery signal series was numerically fitted using a LevenbergMarquardt optimization algorithm for non-linear functions in the MATLAB programming environment (The MathWorks, Natick, MA), to calculate the relaxation time $T_{1}$ for each blood sample. The contrast agent concentrations in blood $C_{b}$ were calculated from $C_{\mathrm{b}}=\left(R_{1}-R_{10}\right) / r_{1}$. Contrast agent concentrations as determined in blood were converted to plasma concentrations by $C_{p}=C_{b} /(1-H c t)$, with an assumed hematocrit value Hct of 0.40 . The plasma concentration time course was numerically fitted to a biexponential function $C_{p}(t)=D\left(a_{1} e^{-t / \tau_{1}}+a_{2} e^{-t / \tau_{2}}\right)$, in which $D$ is the dose (mmol gadolinium/kg body weight), $a_{1}$ and $a_{2}$ are the amplitudes, and $\tau_{1}$ and $\tau_{2}$ the time constants. For the $C_{p}$ used in the simulations, an initial linear rise was added to account for the first pass of the contrast agent, resulting in:

$$
C_{\mathrm{p}}(t)=\left\{\begin{array}{cl}
\frac{t}{t_{1}} D & t<t_{1} \\
D\left(a_{1} \mathrm{e}^{-t / \tau_{1}}+a_{2} \mathrm{e}^{-t / \tau_{2}}\right) & t \geq t_{1}
\end{array}\right.
$$

For the simulations, $t_{1}$ was set to $30 \mathrm{~s}$. 


\section{Reliability analysis}

Monte Carlo simulations $[87,89]$ were performed in MATLAB to investigate the influence of $K^{\text {trans }}$ sampling frequency, relative noise level, and acquisition time on the reliability of pharmacokinetic parameter estimation. Reliability was expressed in terms of bias and variance, where the bias of a pharmacokinetic parameter was defined as the difference of median value of the runs and the input value, and the variance was expressed as the relative difference between 5 and $95 \%$ confidence interval values of the estimated parameter. Parameter estimation was defined to be reliable for bias $<5 \%$, and variance $<10 \%$ with respect to the input value.

A high temporal resolution $(10 \mathrm{~Hz})$ biexponential plasma concentration curve $C_{p}$ was constructed, with magnitudes and time constants as measured in vivo for both the SCA and MCA. This $C_{p}$ was used to numerically calculate the convolution integral for the tissue concentration time curve $C_{t}$, according to equation 4.3. Subsequently, $C_{\mathrm{p}}$ and $C_{\mathrm{t}}$ were undersampled with sampling frequency $f_{\mathrm{s}}$ and Gaussian noise was added, with a noise level relative to the maximum tissue concentration, which is independent of $f_{\mathrm{s}}$ and $T_{\mathrm{acq}}$.

The first Monte Carlo simulation was designed to study the relation between contrast agent molecular size and the reliability of pharmacokinetic parameter estimation. As $K^{\text {trans }}$ is the only parameter that is directly influenced by molecular size (where $K^{\text {trans }}$ increases with decreasing molecular size), the reliability of the pharmacokinetic parameters was calculated for a wide, logarithmically distributed, range of $K^{\text {trans }}$ values $\left(0.001-1 \mathrm{~min}^{-1}\right)$. The default sampling frequency $f_{\mathrm{s}}$ was $10 \mathrm{~min}^{-1}$ and acquisition time $T_{\text {acq }}$ was $15 \mathrm{~min}$. Gaussian noise, with a default noise level of 3 $\%$, related to the concentration maximum in the tissue was added. The noise level was based on previous DCE-MRI measurements in rabbits. The other simulations were performed with $K^{\text {trans }}$ mimicking the leakage rate for an MCA and an SCA in muscle tissue. The values were set to $0.006 \mathrm{~min}^{-1}$ and $0.13 \mathrm{~min}^{-1}$, representing an MCA and an SCA, respectively, while $v_{e}$ and $v_{p}$ were set to 0.09 and 0.015 , respectively $[77,143]$.

In the second simulation the influence of $f_{\mathrm{s}}$ was investigated, so $T_{\text {acq }}$ was kept constant at 15 minutes, and $f_{\mathrm{s}}$ was varied logarithmically $\left(5-60 \mathrm{~min}^{-1}\right)$. The relative noise level was $3 \%$ and was not adjusted for varying $f_{\mathrm{s}}$ to investigate its effect separately. In the third simulation the influence of $T_{\text {acq }}$ was determined, keeping $f_{\mathrm{s}}$ constant at $10 \mathrm{~min}^{-1}$, relative noise level at $3 \%$, and $T_{\text {acq }}$ (with respect to the start on injection) was varied logarithmically (range: $0.5-60 \mathrm{~min}$ ). In the fourth simulation the relative noise level was varied logarithmically (range: $0.5-20 \%$ ), while $T_{\text {acq }}$ was 15 minutes and $f_{\mathrm{s}}$ was $10 \mathrm{~min}^{-1}$. For the fifth simulation, $T_{\text {acq }}$ was 15 minutes, $f_{\mathrm{s}} 10 \mathrm{~min}^{-1}$, and noise level $3 \%$ to determine the co-dependency of the pharmacokinetic parameters. The last four simulations were performed separately for SCA and MCA parameters and the start time for downsampling was randomly chosen between $t=0$ and $t=1 / f_{\mathrm{s}}$ to mimic time offset variations. The number of 
simulation runs $N_{\text {MC }}$ was 1000 .

In each simulation run, the pharmacokinetic parameters were determined by a Levenberg-Marquardt optimization algorithm applied to the undersampled and noisy $C_{p}$ and $C_{t}$. A maximum number of 1000 iterations and function evaluations were allowed. The boundaries of the parameter estimates were set to 0 and 1 for all pharmacokinetic parameters and start values were 0.5 times the input value.

\section{Sampling analysis}

The mechanisms leading to over- or underestimation during undersampling are illustrated in both the time and frequency domain.

Time domain: First, a tissue concentration time curve $C_{t}$ was calculated based on the default pharmacokinetic parameters as described in the previous section and the measured AIF. Subsequently, both $C_{t}$ and $C_{p}$ were sampled at frequencies varying from 1 - $30 \mathrm{~min}^{-1}$. Undersampling was repeated several times with varying starting time (jitter). Typical examples were used to explain why the bias is positive or negative.

Frequency domain: Discrete and continuous forms of $C_{p}(t)$ and $C_{t}(t)$ were Fourier transformed to $C_{p}(\omega)$ and $C_{t}(\omega)$. Based on these curves $H(\omega)$ was calculated. We compared the discrete $H(\omega)$ to the continuous $H(\omega)$ to explain to explain the effect of aliasing on the bias in $K^{\text {trans }}$. 


\subsection{Results}

\section{Arterial input function}

The plasma concentration curves (figure 4.2) after injection of $0.15 \mathrm{mmol} / \mathrm{kg} \mathrm{Gd}$ DTPA or $0.10 \mathrm{mmol} / \mathrm{kg}$ Gadomer were fitted by a biexponential function with the following parameters: $a_{1}$ and $a_{2}$ were 22 and $9.7 \mathrm{~kg} / \mathrm{L}$ for Gd-DTPA and 24 and 5.5 $\mathrm{kg} / \mathrm{L}$ for Gadomer and time constants $\tau_{1}$ and $\tau_{2}$ were 2.5 and $75 \mathrm{~min}$ for Gd-DTPA and 5.5 and 70 min for Gadomer.

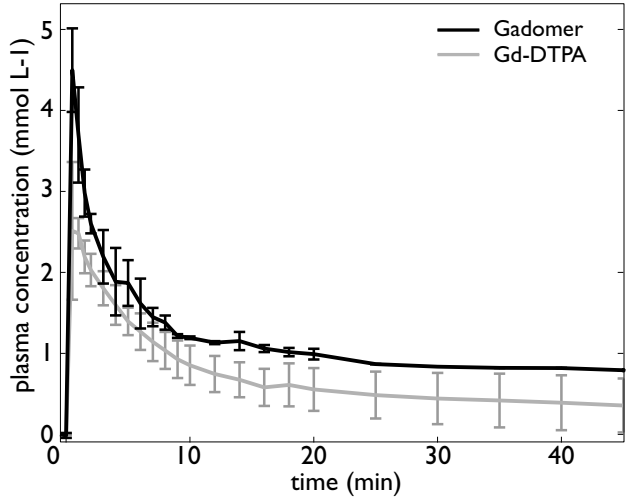

Figure 4.2: Measured plasma concentration time curves after administration of a $0.10 \mathrm{mmol}$ $\mathrm{Gd} / \mathrm{kg}$ Gadomer (grey) and $0.15 \mathrm{mmol} \mathrm{Gd} / \mathrm{kg} \mathrm{Gd}$-DTPA (black). Amplitudes $a_{1}$ and $a_{2}$ were 22 and $9.7 \mathrm{~kg} / \mathrm{L}$ for Gd-DTPA and 24 and $5.5 \mathrm{~kg} / \mathrm{L}$ for Gadomer. Time constants $\tau_{1}$ and $\tau_{2}$ were 2.5 and $75 \mathrm{~min}$ for Gd-DTPA and 5.5 and $70 \mathrm{~min}$ for Gadomer.

\section{Reliability analysis}

The reliability of parameter estimation was highly dependent on $K^{\text {trans }}$ (figure 4.3). The bias and variance of $K^{\text {trans }}$ and $v_{p}$ improved for decreasing $K^{\text {trans }}$ values. The variance in the output $K^{\text {trans }}$ was less than $10 \%$ for $K^{\text {trans }}<0.03 \mathrm{~min}^{-1}$. To obtain a variance of less than $10 \%$ in $v_{\mathrm{p}}, K^{\text {trans }}$ had to be less than $0.012 \mathrm{~min}^{-1}$. The $v_{\mathrm{e}}$ parameter could be determined reliably (variance $<10 \%$ and bias $<5 \%$ ) only for small range of $K^{\text {trans }}$ values $\left(0.005-0.1 \mathrm{~min}^{-1}\right)$. Estimation of $v_{\mathrm{p}}$ became strongly (negatively) biased with large variances for $K^{\text {trans }}>0.01 \mathrm{~min}^{-1}$.

Figure 4.4 shows the bias and variance of the estimated pharmacokinetic parameters as a function of sampling frequency (fig. 4.4A), relative noise level (fig.4.4B), and acquisition time (fig.4.4C) for the SCA and the MCA. A smaller bias and variance for all three pharmacokinetic parameters were obtained for the MCA compared to the SCA. To obtain a $10 \%$ variance in $K^{\text {trans }}, f_{\mathrm{s}}$ for the SCA $\left(28 \mathrm{~min}^{-1}\right)$ needed 

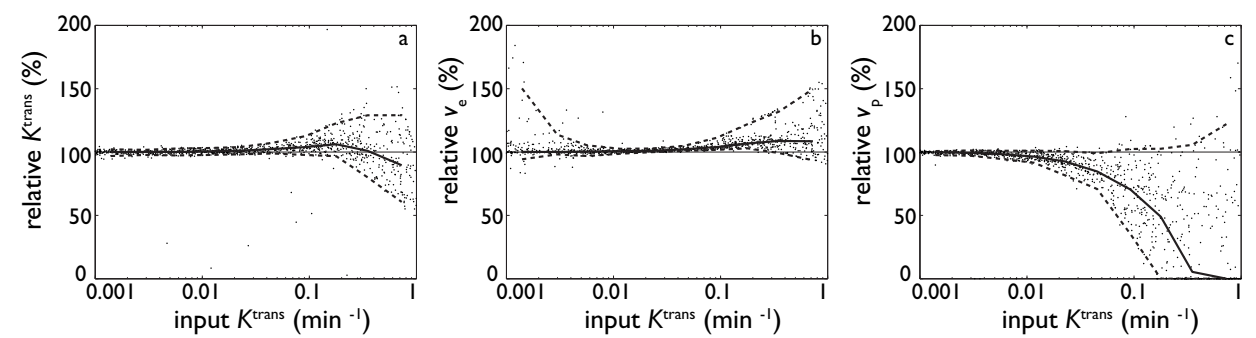

Figure 4.3: Bias and variance in the estimation of $K^{\text {trans }}(a), v_{e}(b)$, and $v_{p}$ (c) for a range of $K^{\text {trans }}$ values. Dots represent the outcome of each Monte Carlo simulation. The solid line is the median and dashed lines represent the 5 and $95 \%$ confidence intervals of the estimates. Input $v_{\mathrm{e}}$ and $v_{\mathrm{p}}$ were 0.09 and 0.015 , respectively. Sample frequency was $10 \mathrm{~min}^{-1}$, relative noise level $3 \%$, and acquisition time was $15 \mathrm{~min}$.

to be 20 times faster than for the MCA $\left(1.3 \mathrm{~min}^{-1}\right)$. For the MCA, $v_{p}$ could be obtained reliably at relatively low $f_{\mathrm{S}}\left(>3.5 \mathrm{~min}^{-1}\right)$, while for SCA a very high $f_{\mathrm{S}}(>$ $\left.60 \mathrm{~min}^{-1}\right)$ ) was required. Bias appeared to have a weak dependency on the relative noise level for all three parameters, whereas variance strongly increased with decreasing noise. For a variance smaller than $10 \%$ in all parameters, the relative noise level should be less than $6 \%$ for the MCA. The same precision in $K^{\text {trans }}$ and $v_{\mathrm{e}}$ with the SCA was achieved with a noise level $<1 \%$. The effect of $T_{\text {acq }}$ on the reliability of $v_{\mathrm{p}}$ was small for both contrast agents. A variance less than $10 \%$ in $K^{\text {trans }}$ and $v_{\mathrm{e}}$ was not reached for the SCA. However, the variance was almost constant for $T_{\text {acq }}>$ $1.7 \mathrm{~min}$. For the MCA, variance decreased significantly for increasing $T_{\text {acq }}$. Reliable estimation was realized for $T_{\text {acq }}>5 \mathrm{~min}$ for $K^{\text {trans }}$ and $T_{\text {acq }}>13 \mathrm{~min}$ for $v_{\mathrm{e}}$.

The parameters $K^{\text {trans }}$ and $v_{e}$, and $K^{\text {trans }}$ and $v_{p}$ (figures $4.5 \mathrm{a}$ and b, respectively) showed a codependency. For the MCA, an overestimation of $K^{\text {trans }}$ was mostly accompanied by an underestimation of $v_{e}$, while for the SCA, $v_{e}$ was usually overestimated. Overestimation of $K^{\text {trans }}$ was generally accompanied by underestimation of $v_{p}$. This effect was most notable for the SCA.

\section{Sampling analysis}

Time domain: The effect of sampling frequency on the $K^{\text {trans }}$ estimation using equation 4.2 can be explained in the time domain as illustrated in figure 4.6. For very low sampling frequencies ( $1 \mathrm{~min}^{-1}$ on the timescale of an SCA) both the discrete version of the derivative $\mathrm{d} C_{t} / \mathrm{dt}$ and $C_{\mathrm{p}}$ will usually be underestimated, but the relative error in the sampled $C_{p}$ is smaller, which will result in a negative bias for $K^{\text {trans }}$. At higher sampling frequencies (> $5 \mathrm{~min}^{-1}$ on the SCA timescale), however, the estimation of $\mathrm{d} C_{t} / d t$ is quite accurate and the underestimation of $C_{p}$ is relatively the largest, which causes overestimation of Ktrans. All sampling frequencies in the studied range were relatively fast with respect to the timescale of an MCA. In this 


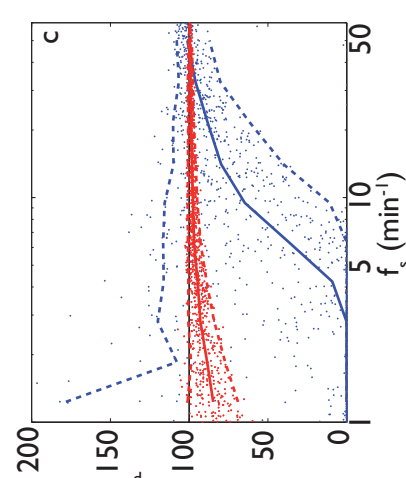

(\%) ${ }^{d} \wedge$ әм!џерәд

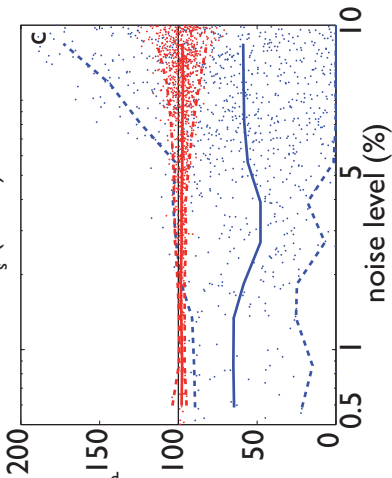

(\%) ${ }^{\mathrm{\Lambda}} \wedge$ әліперәд

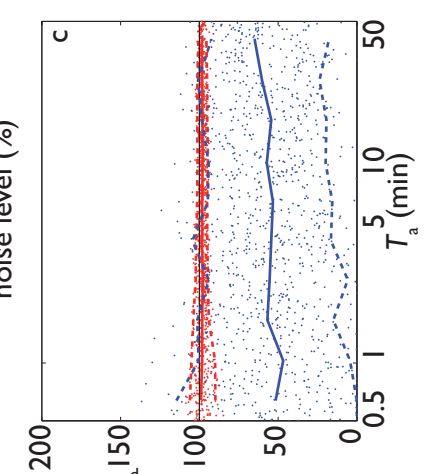

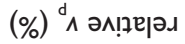

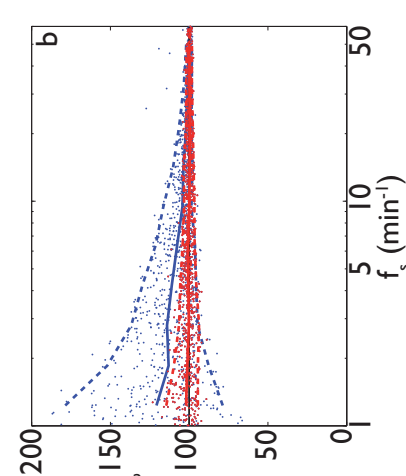

$(\overline{\%}){ }^{2} \wedge$ әм!ฺщер

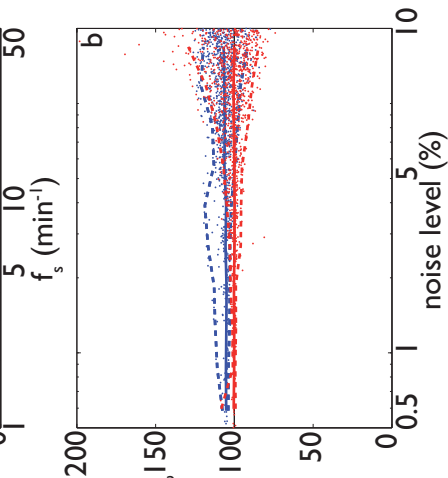

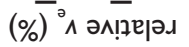

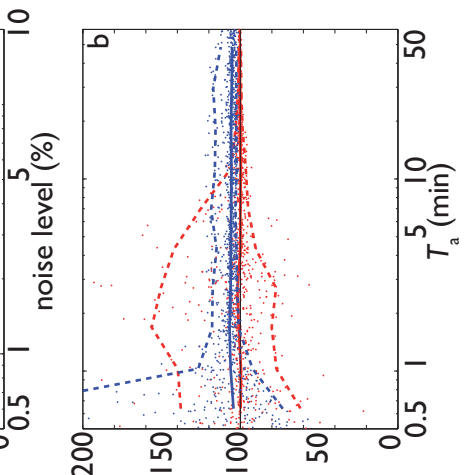

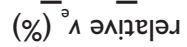
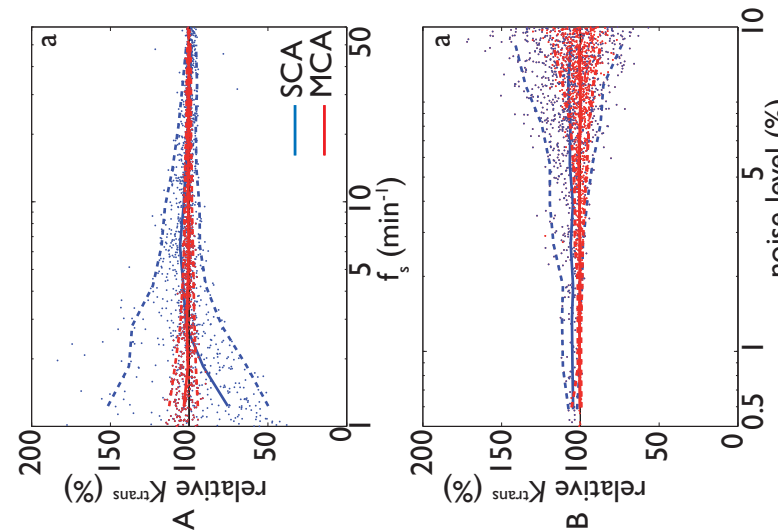

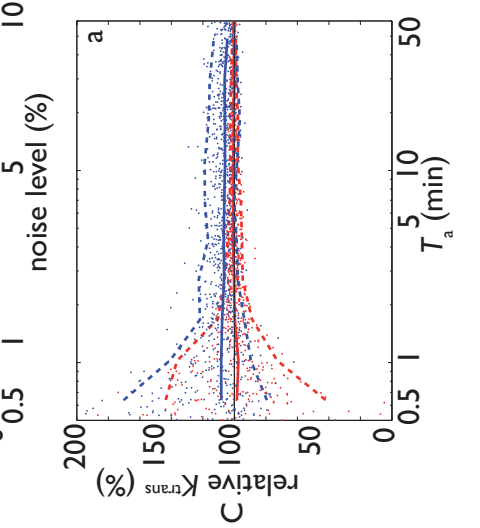

Figure 4.4: Bias and variance in estimation of $K^{\text {trans }}(a), v_{e}(b)$ and $v_{p}$ (c) for varying sampling frequency $f_{\mathrm{s}}(\mathrm{A})$, noise level $(\mathrm{B})$, and acquisition time $T_{\text {acq }}(\mathrm{C})$ for a small contrast agent (blue, $K^{\text {trans }}=0.13 \mathrm{~min}^{-1}$ ) and a medium sized contrast agent (red, $K^{\text {trans }}=0.006$ $\min ^{-1}$ ). Dots represent the result of each Monte Carlo simulation using 1000 runs; solid line is the median and dashed lines represent the 5 and $95 \%$ confidence intervals of the estimates. The default $f_{\mathrm{s}}$ was $10 \mathrm{~min}^{-1}$, noise level $3 \%$, and $T_{\text {acq }} 15 \mathrm{~min}$. 

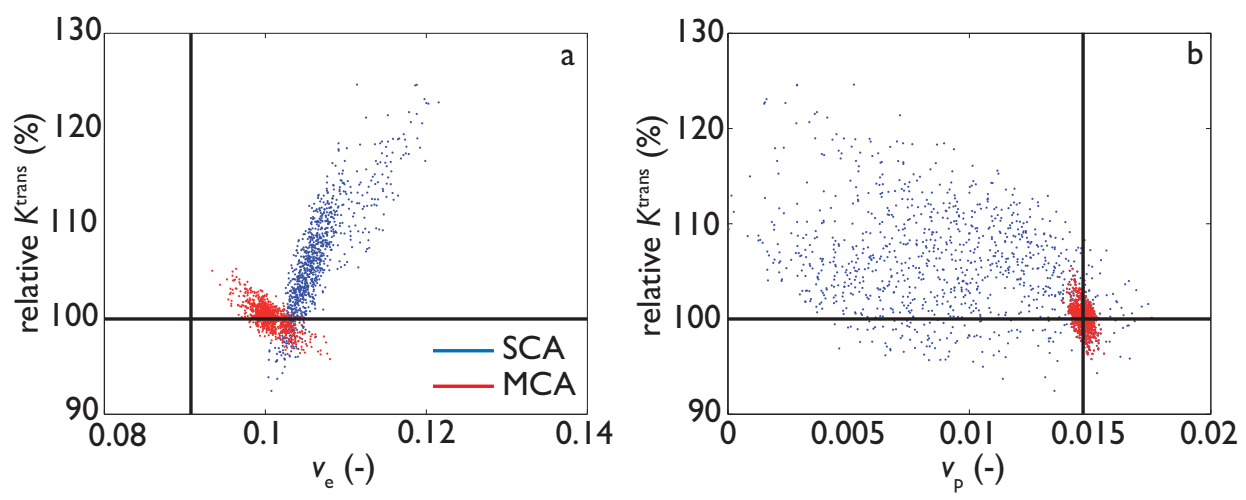

Figure 4.5: Co-dependency of $K^{\text {trans }}$ and $v_{e}(a)$, and $K^{\text {trans }}$ and $v_{p}$ (b) for the SCA (blue) and MCA (red). The MCA provides a smaller uncertainty for each parameter compared to SCA. A tilted (i.e. non-orthogonal) cloud of scattered data points indicates co-dependency of parameters. For the MCA, the uncertainties in $v_{\mathrm{e}}$ and $K^{\text {trans }}$ are negatively correlated, which means that an underestimated $v_{e}$ does in general provide a slightly overestimated $K^{\text {trans }}$ (and vice versa). For the SCA, an underestimated $v_{\mathrm{p}}$ provides may yield a strongly overestimated $K^{\text {trans }}$ (and vice versa).

case, the largest error was made in the estimation of $C_{p}$ (not shown), resulting in overestimation of $K^{\text {trans }}$.

Frequency domain: The effect of the sampling frequency on the sampled $C_{t}$ and $C_{p}$ for the SCA is shown in figure 4.7 for $f_{s}$. Fourier components of $C_{t}$ and $C_{p}$ at frequencies higher than the Nyquist frequency (i.e. $2 f_{\max }$ ) are not properly measured, but cause aliasing of the Fourier components with lower frequencies. Especially the frequencies just below the Nyquist frequency become positively biased. In general, this aliasing effect on $C_{p}$ is stronger than on $C_{t}$, because $C_{p}$ usually contains stronger high frequency components. During pharmacokinetic modeling the discrete version of $H(\omega)$ is calculated from the ratio of the discrete versions of $C_{t}$ and $C_{p}$, which will be affected by this aliasing effect. The effect on the transfer function is that the $3 \mathrm{~dB}$ attenuation frequency $\omega_{\mathrm{p}}$ shifts to a lower frequency value. Provided that the estimation of the low frequency plateau is accurate, this will result in an underestimation (i.e. negative bias) of $K^{\text {trans }}$. The steeper slope is consistent with a lower $v_{p}$. 

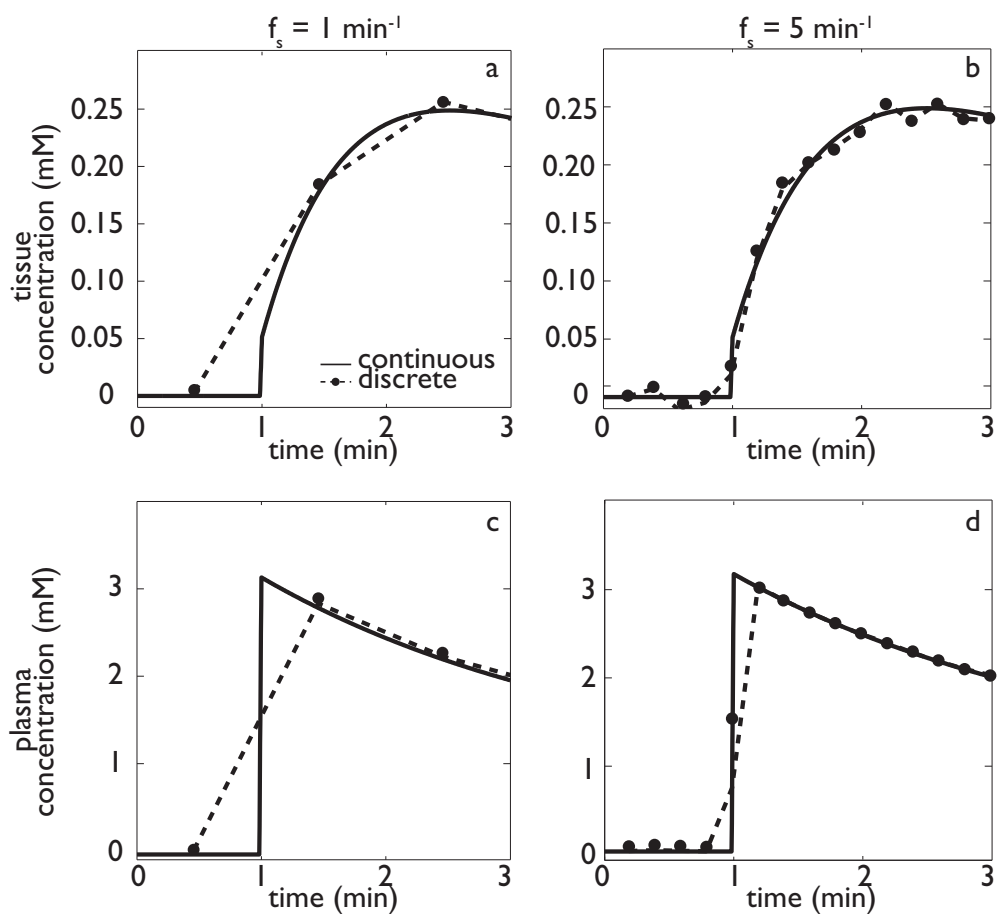

Figure 4.6: Sampling of the tissue ( $a$ and $b$ ) and plasma ( $c$ and $d$ ) concentration curves for low sampling frequencies ( $a$ and $\mathrm{c}: 1 \mathrm{~min}^{-1}, \mathrm{~b}$ and $\mathrm{d}: 5 \mathrm{~min}^{-1}$ ). The continuous functions are shown, as are the corresponding sampled signals with added noise. Note that the initial slopes of the plasma and tissue concentration curves are underestimated more strongly with the low temporal resolution sample signals $\left(f_{\mathrm{s}}=1 \mathrm{~min}^{-1}\right)$ than with the higher sampled signals $\left(f_{\mathrm{s}}=5 \mathrm{~min}^{-1}\right)$. The timescale is based on a small contrast agent. 


\subsection{Discussion}

\section{Current findings}

In this study the reliability of pharmacokinetic parameter estimation of SCAs and MCAs in muscle tissue were compared. To this end, the mathematical structure of the generalized pharmacokinetic model including a vascular component, and the influence of undersampling in both the time and frequency domain were studied. Monte Carlo simulations were performed to investigate the relation between the reliability of pharmacokinetic parameters and the sampling frequency, acquisition time, and noise level. The MCA appeared to be superior to the SCA in terms of bias and variance of the estimated pharmacokinetic parameters $K^{\text {trans }}, v_{e}$, and $v_{p}$ that describe the tissue's microvascular environment. Reliable estimates of $K^{\text {trans }}$ were obtained at lower sampling frequencies and $v_{p}$ could be determined reliably with the MCA. This was not possible for the SCA.
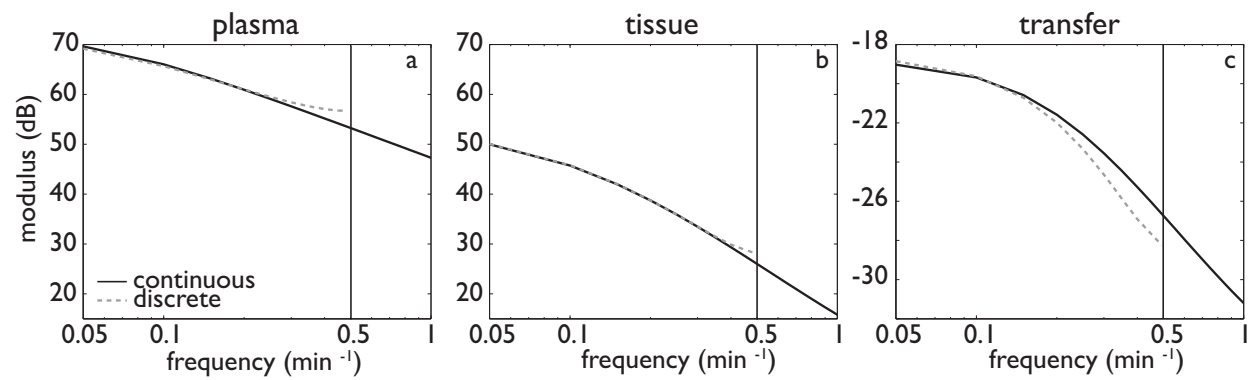

Figure 4.7: Modulus of plasma concentration $C_{p}$ (panel a), tissue concentration $C_{t}(b)$, and tissue transfer $H(c)$ in the frequency domain. Solid lines represent the original continuous functions, and dashed lines the discrete function. Sampling frequency was $1 \mathrm{~min}^{-1}$, so the highest frequency represented in the sampled discrete signal was $0.5 \mathrm{~min}^{-1}$. Note the aliasing effect just below the highest frequency in the sampled $C_{p}$ and $C_{t}$. The estimated discrete transfer function $H$ showed a lower frequency transfer compared to the continuous $H$.

\section{Frequency analysis}

Reliable estimation of pharmacokinetic parameters requires sampling of all relevant frequencies. Both the pole and zero frequency contain information on $K^{\text {trans }}$ and should be included. Because the slower extravasation of an MCA, the tissue transfer function shifts towards the lower frequencies, lower sampling rates and longer acquisition times are required than for an SCA. The parameter $v_{p}$ is defined by the high frequency plateau in the tissue transfer function. For the SCA, this plateau is reached at a higher frequency compared with the MCA. Estimation of $v_{p}$ improves 
for higher sampling frequencies for the SCA as well as for the MCA, but for the SCA estimation remains imprecise even at a very high sampling rate. Reliable estimation of $v_{p}$ is only achieved using an MCA. To optimally estimate $v_{e}$, the smallest discrete frequency that is acquired, $f_{\min }=1 / T_{\text {acq }}$, needs to be low enough to contain information about the low frequency plateau that defines. While for an SCA this requirement is met at acquisition times as short as 1 - 2 minutes, an MCA required a much longer acquisition time, especially for the estimation of $v_{e}(>13 \mathrm{~min})$.

A negative co-dependency of $K^{\text {trans }}$ and $v_{e}$ was found for the MCA, due to the fact that when both the plateau and pole frequency are excluded for the sampled range, an underestimation of the plateau (i.e. underestimation of $v_{e}$ ) is most often accompanied by a shift towards the higher frequencies, causing an overestimation of $K^{\text {trans }}$. However, when both the pole frequency and the plateau were acquired, as was the case for the SCA, an overestimation of $K^{\text {trans }}$ is followed by an overestimation of $v_{e}$, because $\omega_{p}=\frac{K^{\text {trans }}}{v_{e}}$. Underestimation of $K^{\text {trans }}$ is frequently accompanied by overestimation of $v_{p}$, because both $K^{\text {trans }}$ and $v_{p}$ are related to the initial change in tissue concentration. The instant rise in $C_{t}$ at the arrival of the contrast agents reflects the plasma fraction $v_{p}$, and the initial steepness $\mathrm{d} C_{t}\left(0^{+}\right) / d t$ equals $K^{\text {trans }}$. This confounding effect is less pronounced for the MCA, because the initial fast rise is mainly caused by a concentration change in the vascular space and can easily be distinguished from the more gradual increase due to leakage into the extracellular extravascular space.

\section{Implications for experimental set-up}

This study shows that a priori knowledge of the pharmacokinetic parameters in the tissue of interest can be used to optimize experimental set-up. Of course, true values of the parameters are not known beforehand. Practically, the largest variation is found in $K^{\text {trans }}$, where the primary determinants are contrast agent size and tissue of interest (muscle or tumor). Results from previous studies with the same contrast agent and tissue can be used to predict the range of expected parameter values.

When a lower sampling frequency is applied using an MCA, imaging can benefit from the gain in acquisition time per dynamic phase in several ways. A higher spatial resolution gives the opportunity to study tissue vascular properties in more detail. One should, however, consider the increase in noise and its detrimental effect on precision (variance) in parameter estimation. This effect is relatively the largest for an MCA, as the initial rise in tissue concentration is small with respect to the noise level. Another way to benefit from longer dynamic acquisition times is decreasing the noise level by signal averaging. This improves the reliability of parameter estimation, especially for an MCA. 
When using an SCA, all relevant information is acquired within the first five minutes after contrast agent administration. The use of MCAs may increase patient discomfort and body motion artifacts, as the acquisition time that is required for optimal parameter estimation is 2 - 3 times longer than for the SCA.

\section{Physiological interpretation}

Although the higher reliability might favor the use of an MCA instead of an SCA, it should be noted that the physiological interpretation of the pharmacokinetic parameters is quite different. For an SCA, the rate at which the interstitial concentration increases is highly determined by the amount of blood supply (flow-limited) and not by the permeability of the vasculature, whereas for an MCA this rate is more dependent of the permeability characteristics of the vessel wall with respect to the contrast agent size $[139,144]$. In tissue with hyperpermeable microvessels (e.g. tumors or inflammations), tissue enhancement by MCAs is more specific and largely reflects permeability, where SCAs mainly provides information on microvascular flow. Therefore, it depends on the microvascular property that is of interest to judge which contrast agent molecule is most adequate.

\section{Limitations}

The current results were obtained using computer simulations and assumed ideal time-course data to which Gaussian noise was added. In other words, the data used did not suffer from signal degrading effects as may occur with in vivo data. As other sources of errors may add to the uncertainty of the estimated parameters, it should therefore be emphasized that the current results may represent best case scenarios. The bias and variance presented should therefore be regarded as lower boundaries when compared to in vivo measurements. However, we feel that with these ideal data provide valuable insights on the influence of several controllable imaging parameters including sampling frequency, acquisition time, and signal-to-noise characteristics on the estimation of pharmacokinetic parameters.

Validation of this simulation study is not straightforward and has pitfalls. The main advantage of Monte Carlo simulations is that a large number of independent estimations can be accomplished. In vivo, this could be approximated by calculating the standard deviation for multiple voxels in the same muscle. However, the tissue will not be completely homogeneous and the voxels are not independent. Moreover, comparing various acquisition settings would still require many contrast injections, preferably within the same animal during the same session, which is practically and ethically not realizable. This problem could be solved by virtually decreasing the acquisition time and sampling frequency of an in vivo dataset, by omitting specific dynamic phases. However, these virtual datasets are not independent. We feel the 
best way to validate the relations between pharmacokinetic parameters and measurement settings described in this study is to use a $2 \times 2$ design, in which two different sampling frequencies and two different contrast agents are applied to different groups of similar subjects or animals.

\section{Conclusions}

In pharmacokinetic modeling, an MCA such as Gadomer theoretically provides improved reliability of pharmacokinetic parameter estimation compared to an SCA such as Gd-DTPA, due to the lower leakage rate of MCA into the tissue. The MCA allowed lower sampling frequencies compared to an SCA, while obtaining higher reliability for the determination of the transfer constant $K^{\text {trans }}$. Precise determination of the fractional plasma volume $v_{p}$ can be obtained at a much lower frequency for an MCA than for an SCA. 

Optimized pharmacokinetic modeling for the detection of perfusion differences in skeletal muscle with DCE-MRI: effect of contrast agent size 


\section{Abstract}

Purpose: The goal of this study was to optimize dynamic contrast-enhanced (DCE) MRI analysis for differently sized contrast agents and to evaluate the sensitivity for microvascular differences in skeletal muscle.

Methods: In rabbits, pathophysiological perfusion differences between hind limbs were induced by unilateral femoral artery ligation. On days 14 and 21, DCE-MRI was performed using a medium-sized (MCA, Gadomer) or small contrast agent (SCA, Gd-DTPA). Acquisition protocols were adapted to the pharmacokinetic properties of the contrast agent. Model-based data analysis was optimized by selecting the optimal model considering fit error, estimation uncertainty and parameter interdependency from three two-compartment pharmacokinetic models (normal and extended generalized kinetic model, and Patlak model). Model-based parameters were compared to the model-free parameter area-under-curve (AUC). Finally, the sensitivity of transfer constant $K^{\text {trans }}$ and $A \cup C$ for physiological and pathophysiological microvascular differences was evaluated.

Results: For the MCA, the optimal model included $K^{\text {trans }}$ and plasma fraction $v_{p}$. For the SCA,$K^{\text {trans }}$ and interstitial fraction $v_{e}$, should be incorporated. For the MCA, $K^{\text {trans }}$ was $4.8 \pm 0.210^{-3} \mathrm{~min}^{-1}$ (mean \pm standard error) and $3.6 \pm 0.110^{-3} \mathrm{~min}^{-1}$ for the red soleus and white tibialis muscle, respectively, $p<0.01$. With the SCA, $K^{\text {trans }}$ was $81 \pm 510^{-3} \mathrm{~min}^{-1}$ (soleus) and $66 \pm 510^{-3} \mathrm{~min}^{-1}$ (tibialis), $p<0.01$. In the ischemic limb $K^{\text {trans }}$ was significantly decreased relative to the control limb (soleus: $15-20 \%$; tibialis: $5-10 \%$ ). Similar differences in AUC were found for both contrast agents.

Conclusions: For optimal estimation of microvascular parameters, both modelbased and model-free analysis should be adapted to the pharmacokinetic properties of the contrast agent. The detection of microvascular differences based both $K^{\text {trans }}$ and $A \cup C$ was most sensitive when the analysis strategy was tailored to the contrast agent used. The MCA was equally sensitive for microvascular differences as the SCA, with the advantage of improved spatial resolution.

Published in Medical Physics 2010 


\subsection{Introduction}

Several investigators have proposed neovascularization as a therapeutic strategy aimed to relieve impaired tissue perfusion due to atherosclerotic occlusive disease in the coronary and peripheral arteries [94, 117, 145]. However, development of optimized treatment strategies is hampered by a lack of suitable non-invasive methods to assess the effect of different treatment regimens [39].

A particularly promising method for non-invasively monitoring changes in endorgan perfusion is dynamic contrast-enhanced magnetic resonance imaging (DCE$\mathrm{MRI}$ ). This technique is based on measuring temporal signal changes caused by passage and extravasation of a contrast agent. The utility of DCE-MRI derives from the assumption that the extravasation rate reflects underlying physiological properties, including microvascular flow and permeability. DCE-MRI already is an established method in oncology $[15,68,71,146]$, and has also proven to be of value in evaluation of myocardial $[147,148]$ and skeletal muscle microvasculature $[76-78,80]$, as DCE-MRI-related parameters correlate well with tissue perfusion measured with microspheres $[85,86]$.

Most DCE-MRI studies have used clinically approved small contrast agents (SCAs) such as gadopentetate dimeglumine (Gd-DTPA; molecular weight: $0.5 \mathrm{kDa}$ ) despite the disadvantage of rapid extravasation and need for fast imaging protocols [87-89]. Medium-sized contrast agents (MCAs) such as Gadomer (apparent molecular weight: $32 \mathrm{kDa}$ [108]) may overcome these problems, because their lower extravasation rates allow longer dynamic acquisition times [112]. The resulting improvement in image quality may facilitate delineation of muscle groups. A relatively unexplored issue remains whether data analysis methods should be adapted to the properties of the contrast agent.

Model-free approaches, such as calculating the area-under-curve (AUC), are relatively independent of injection protocols [79] and highly reproducible [80]. However, since the underlying physiological mechanisms remain undefined, the interpretation of model-free parameters remains ambiguous and depends on the method and contrast agent properties. By describing the mechanisms influencing extravasation in pharmacokinetic models and fitting the data to such models, parameters reflecting physiological properties can be obtained. To this end, a number of pharmacokinetic models, varying in complexity, have been developed. The most frequently used model in studies with SCAs is the Generalized Kinetic Model (GKM) as described by Tofts et al. [83]. It defines the relation between plasma and tissue concentration time curves $\left(C_{\mathrm{p}}\right.$ and $C_{\mathrm{t}}$, respectively) in terms of transfer rate $K^{\text {trans }}$ and volume fraction of the extracellular extravascular space $v_{e}$. However, the GKM neglects the contribution of the concentration of the plasma space $v_{p}$, which is included in the extended GKM [82]. A third model, the Patlak model, is a graphic technique that comes from the fields of computed tomography and nuclear medicine [84]. This model accounts for the contribution of intravascular contrast agent concentration with plasma vol- 


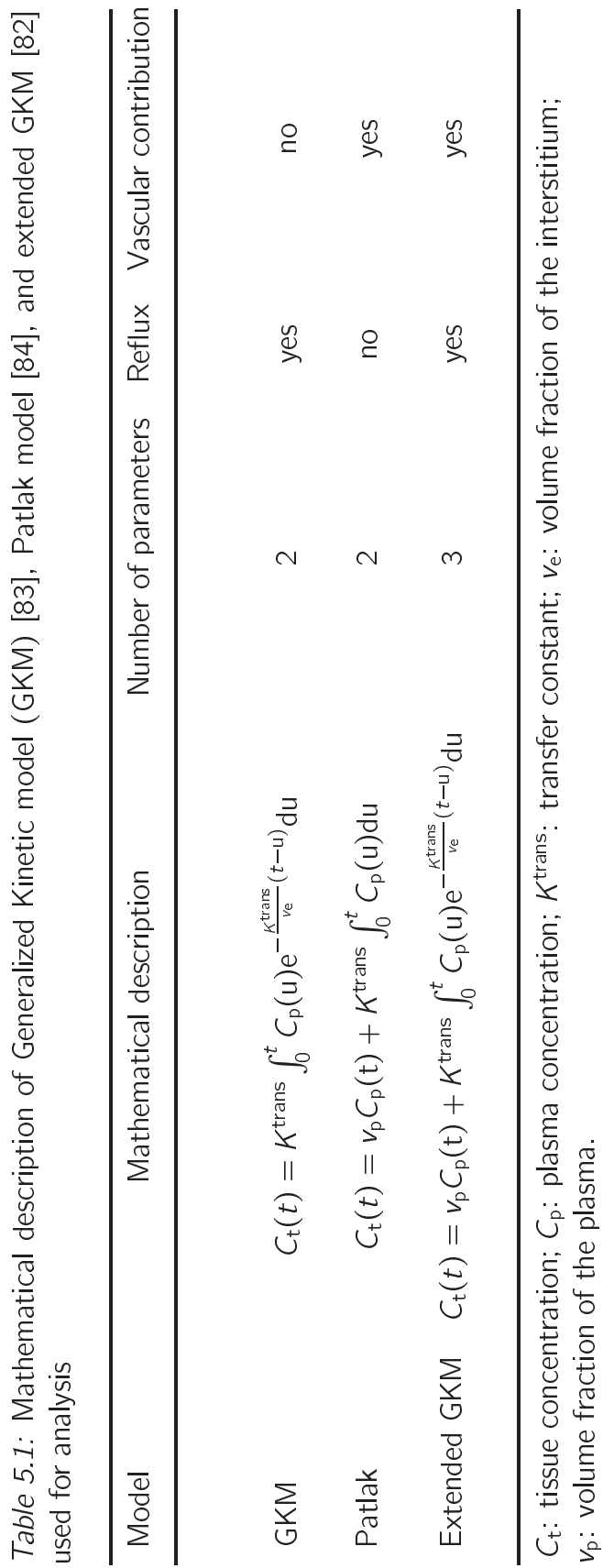


ume fraction $v_{p}$, but neglects reflux (i.e. $k=K^{\text {trans }} / v_{e}=0$ ). An overview of the three models including their mathematical descriptions is provided in table 5.1.

Many studies use one model to compare SCAs and MCAs [74, 144, 149] for reasons of unification. However, since contrast agent size strongly influences the concentration time course, model requirements may also vary among contrast agents. Using an extensive model may seem to be the fairest way for comparisons, yet more elaborate models do not necessarily produce a better fit [150] or more accurate parameters [140]. Assessment of the true merit of a novel contrast agent might therefore require the use of the most appropriate model for a specific setting.

The purpose of this study was to critically select optimized methods for modelbased and model-free data analysis for both an MCA and SCA separately. Extending on previous research, model selection comprised analysis of fit errors, parameter uncertainty and error interdependency. The sensitivity for differences in microvasculature in a rabbit model exhibiting decreased muscle perfusion was determined for both optimal and unified data analysis. 


\subsection{Materials and Methods}

\section{Animal model}

This study was approved by the local animal ethics committee. Six New Zealand White rabbits (weight: approximately $3 \mathrm{~kg}$ ) were included. To simulate the reduced muscle perfusion observed in patients with peripheral arterial occlusive disease, the right femoral artery was ligated as described by Hoefer et al [151]. Due to changes in shear stress, pre-existing arterioles will develop into functional collateral arteries that partially compensate for the loss of blood supply distal to the occlusion [152]. Pathophysiological perfusion differences between the two limbs were thus induced in addition to intrinsic physiological perfusion differences between red (soleus) and white (tibialis anterior) muscle groups.

Anesthesia was induced by intramuscular injection of ketamine hydrochloride and xylazin (50 and $8 \mathrm{mg} / \mathrm{kg}$ body weight, respectively). During the procedure, the rabbits were intubated and ventilated with isofluorane (2-3\%) and oxygen. A sham operation was performed on the contralateral limb, serving as a control. On days 14 and 21 post ligation the rabbits underwent MRI. It was assumed that differences between days 14 and 21 are marginal [32], allowing direct comparison of the two contrast agents in each animal. Venous access was obtained by two canulas placed in the ear vein for separate administration of contrast agent and vasodilator.

\section{Image acquisition}

Animals were imaged in supine position on a clinical 3.0 Tesla MR system equipped with a 5-element phased-array cardiac coil (Philips Medical Systems, Best, The Netherlands). The DCE-MRI protocol included a dynamic $T_{1}$-weighted 3D fast gradient echo sequence. Each animal received an MCA (Gadomer, Bayer Schering Pharma AG, Berlin, Germany) and an SCA (Gd-DTPA) on separate days. Randomization determined which contrast agent was administered first. As the two contrast agents have different pharmacokinetic properties, sampling requirements for DCE-MRI also differ [112]. The MRI protocol was therefore adapted for each contrast agent separately, resulting in the acquisition parameters listed in table 5.2. During DCE-MRI, adenosine $(200 \mu \mathrm{g} / \mathrm{kg} / \mathrm{min})$ was administered intravenously to invoke standardized increased muscle perfusion due to vasodilatation [153]. After acquiring several dynamic pre-contrast phases, the contrast agent (dose: 0.10 and $0.15 \mathrm{mmol} / \mathrm{kg}$ for MCA and SCA, respectively) was injected manually at $0.1 \mathrm{~mL} / \mathrm{s}$ and flushed with 2 $\mathrm{mL}$ saline.

Prior to contrast administration a variable flip angle series $(F A=1,2,4,6,8$, $\left.10,15,20,25^{\circ}\right)$ was acquired to determine pre-contrast $R_{1}\left(R_{1 \text {, pre }}\right)$ by fitting the data to the linearized relation between signal intensity $S$ and $F A[154,155]$ : 
Table 5.2: Acquisition parameters for DCE-MRI with a medium-sized and small contrast agent (MCA and SCA, respectively)

\begin{tabular}{lcc}
\hline & MCA & SCA \\
\cline { 2 - 3 } Matrix & $304 \times 304 \times 60$ & $128 \times 128 \times 30$ \\
Voxel size $\left(\mathrm{mm}^{3}\right)$ & $0.8 \times 0.7 \times 1.3$ & $2.0 \times 1.6 \times 2.5$ \\
Repetition time $(\mathrm{ms})$ & 7.7 & 6.1 \\
Echo time $(\mathrm{ms})$ & 2.0 & 1.0 \\
Flip angle $\left(^{\circ}\right)$ & 20 & 20 \\
Acquisition time per phase $(\mathrm{s})$ & 22.3 & 3.9 \\
Number of pre-contrast phases & 4 & 20 \\
Number of dynamic phases & 35 & 200 \\
\hline
\end{tabular}

$$
\frac{S(F A)}{\sin (F A)}=e^{-T R \cdot R_{1}} \frac{S(F A)}{\tan (F A)}+M \cdot\left(1-e^{-T R \cdot R_{1}}\right)
$$

with $M$ the fitted magnetization. Voxel size and matrix were equal to the dynamic images. TR and TE were 7.7 and $2.0 \mathrm{~ms}$, respectively. The number of signal averages was 4 for the SCA and 2 for the MCA. Acquisition time for the complete series was $1.5 \mathrm{~min}$ for the SCA and $6 \mathrm{~min}$ for the MCA. Immediately following DCE-MRI, the variable flip angle series was repeated to obtain post contrast $R_{1}\left(R_{1, \text { post }}\right)$. The changes in concentration during the acquisition of the series were assumed to be small compared to the changes during the DCE-MRI acquisition.

\section{Image analysis}

Regions of interest (ROIs) were defined within the soleus and tibialis anterior in both the ischemic and control limb on the pre-contrast images. Due to evident differences in spatial resolution between the MCA and SCA images, the observer was not blinded for contrast agent. The signal time curves in the ROls were converted to tissue concentration time curves $\left(C_{t}\right)$ in MATLAB (The MathWorks, Natick, MA) using a linear approximation [78]:

$$
C_{\mathrm{t}}=\frac{\Omega_{C A}}{r_{1, C A}} \cdot\left(S(t)-S_{\text {pre }}\right)
$$

where calibration constant $\Omega_{\mathrm{CA}}$ is determined by $\Omega_{\mathrm{CA}}=\frac{R_{1 \text { post }}-R_{\text {pre }}}{S_{\text {post }}-S_{\text {pre }}}, S(\mathrm{t})$ is signal time curve, and $S_{\text {pre }}$ and $S_{\text {post }}$ are pre- and post-contrast signal intensities, respectively. The contrast agent $T_{1}$ relaxivity $r_{1, C A}$ in plasma at 3 Tesla was taken from literature $\left(r_{1, \text { Gadomer }}=13 \mathrm{~L} \mathrm{mmol}^{-1} \mathrm{~s}^{-1} ; r_{1, \text { Gd-DTPA }}=3.7 \mathrm{~L}^{-1} \mathrm{~s}^{-1}[100]\right)$, and used as an approximation for contrast agent relaxivity in muscle tissue. 


\section{Parameter estimation}

AUC (unit: $\mathrm{s} \mathrm{mmol} / \mathrm{L}$ ) was determined by taking the integral of $C_{\mathrm{t}}(\mathrm{t})$ over a given time period $T_{A \cup C}(1.5,3,6$ and $12 \mathrm{~min})$ after contrast injection. Pharmacokinetic modeling was performed with three different two-compartment models: the conventional and extended generalized kinetic model (GKM) and the Patlak model. A more detailed description and comparison of the models is provided in table 5.1. For all models the analytical solution for $C_{t}(t)$ was calculated, assuming a biexponential $C_{p}(t)$ defined by

$$
C_{p}(t)=D\left(A_{1} \mathrm{e}^{-t / \tau_{1}}+A_{2} \mathrm{e}^{-t / \tau_{2}}\right)
$$

where $D$ is the administered dose (unit: $\mathrm{mmol} / \mathrm{kg}$ ). Amplitudes $A$ and time constants $\tau$ were determined by blood sampling in the femoral artery, as described in a previous experiment 21. $A_{1}$ and $A_{2}$ were found to be 22 and $9.7 \mathrm{~kg} / \mathrm{L}$ for the SCA and 24 and $5.5 \mathrm{~kg} / \mathrm{L}$ for the MCA. $\tau_{1}$ and $\tau_{2}$ were 2.5 and $75 \mathrm{~min}$ for the SCA and 5.5 and $70 \mathrm{~min}$ for the MCA. Differences in bolus arrival time were corrected for by synchronizing $C_{t}$ with the start of tissue enhancement.

Pharmacokinetic parameters were estimated by fitting the measured concentration time curve with a non-linear Gauss-Newton sum-of-least-squares optimization algorithm in MATLAB. A maximum number of 1000 iterations and function evaluations were allowed. Lower and upper boundaries of $K^{\text {trans }}$ were set to 0 and $1 \mathrm{~min}^{-1}$, respectively, and for both $v_{\mathrm{e}}$ and $v_{\mathrm{p}}$ to 0 and $100 \%$.

\section{Model performance}

The optimal model was chosen for each contrast agent separately, considering quality of the fit, uncertainty in parameter estimation and error interdependency between parameters. The sum of squared residuals (SS) were calculated for the control tibialis muscle by:

$$
\mathrm{SS}=\sum\left(C_{\mathrm{t}, \mathrm{fit}}-C_{\mathrm{t}}\right)^{2}
$$

where $C_{t \text {,fit }}$ is the fitted tissue concentration curve, $C_{t}$ the original curve and the summing runs over all discrete time-points of sampling. SS were normalized to relative fit errors (RFE) by taking the square root of SS divided by the squared sum of $C_{\mathrm{t}}$ :

$$
\mathrm{RFE}=\sqrt{\frac{\sum\left(C_{\mathrm{t}, \mathrm{fit}}-C_{\mathrm{t}}\right)^{2}}{\sum C_{\mathrm{t}}^{2}}}
$$

Parameter estimation uncertainty and interdependency were derived from the 
covariance matrix, whose diagonal elements represent estimation uncertainty, and off-diagonal elements indicate parameter interdependencies.

\section{Image quality}

Maximal signal enhancement $\left(E_{\max }\right)$ was calculated with:

$$
E_{\max }=\frac{S_{\max }-S_{\text {pre }}}{S_{\text {pre }}} \cdot 100 \%
$$

where $S_{\max }$ is the maximum signal intensity in $C_{\mathrm{t}}(\mathrm{t})$. Two subsequently acquired pre-contrast images were used to calculate the signal-to-noise-ratio (SNR): SNR = $\frac{\mathrm{S}_{\text {pre }} \sqrt{2}}{\mathrm{SD}_{\text {sub }}}$, where $S D_{\text {sub }}$ is the standard deviation in a muscle region in the subtracted image. The noise level on $C_{t}$ was approximated by using equation 5.4 over the relatively slowly varying part of $C_{t}$.

\section{Statistics}

Statistical analysis was performed with commercial software (SPSS, Chicago, III, version 17.0). Analysis of variance (ANOVA) was performed to rule out differences in $K^{\text {trans }}$ between day 14 and 21 .

F-tests were used to test which of the two 2-parameter models was superior in terms of SS, and to assess whether expansion to the 3-parameter model gave a significantly improvement in SS for compared to the best 2-parameter model. In the latter case, the $F$-statistic was calculated according to the extra sum of squares method $[156,157]$ :

$$
F_{d f_{3}-d f_{2}}=\frac{\left(\mathrm{SS}_{2}-\mathrm{SS}_{3}\right) /\left(d f_{2}-d f_{3}\right)}{\mathrm{SS}_{3} / d f_{3}}
$$

where $\mathrm{SS}_{n}$ and $d f_{n}$ were the SS and degrees of freedom for the model with $n$ parameters ( $n=2$ or 3 ), respectively.

Differences between models, quantified by means of estimation uncertainties and parameter interdependencies, were tested with paired Wilcoxon signed-rank tests. Multivariate linear regression was used to determine the relative contributions of the physiological properties $K^{\text {trans }}$ and $v_{e}$ or $v_{p}$ to AUC. Differences between red and white, and ischemic and control muscle groups were tested using two-sided paired Student's $t$-tests. Differences in image quality in terms of $E_{\max }$, SNR and noise level were tested with independent sample Student's $t$-tests. Effects and differences were considered significant when $p<0.05$. 


\subsection{Results}

Tissue concentration time curves for the MCA showed a distinctive instant increase representing bolus arrival in the vascular space (figure 5.1). For the SCA this rise was indiscernible from the rapid increase caused by extravasation of the contrast agent.
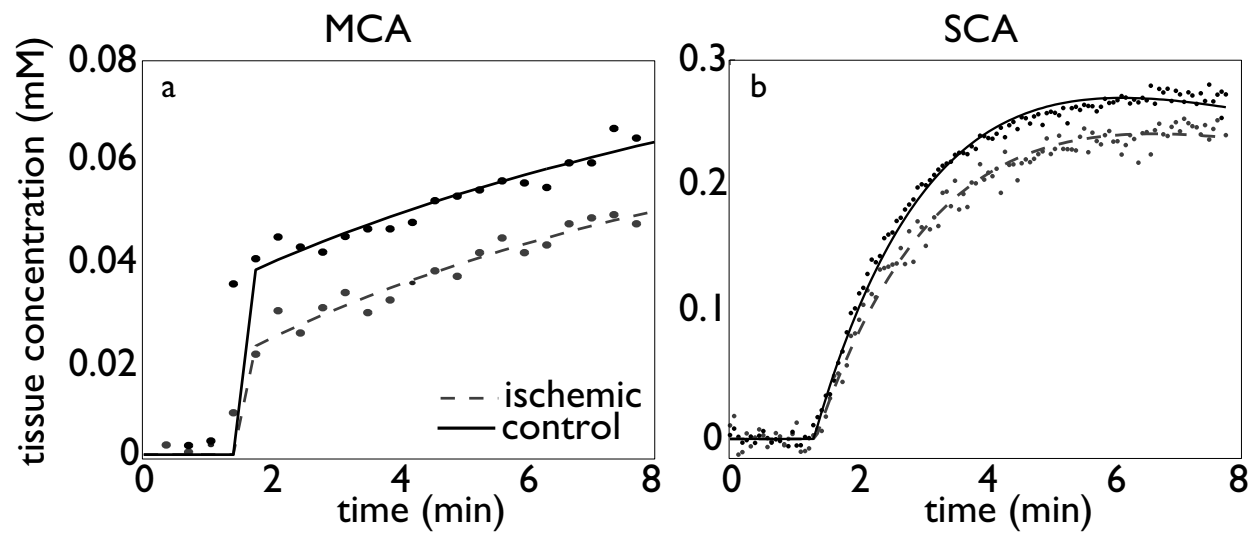

Figure 5.1: Tissue concentration time curves of the MCA (panel a) and the SCA (panel b) in the tibialis anterior muscle of the ischemic (gray, dashed) and control limb (black, solid). Dots represent measured raw DCE-MRI data and the curves represent the fits with the optimal pharmacokinetic model. For the MCA, the Patlak model was used, whereas the SCA curve was fitted with the generalized kinetic model.

\section{Model-based approach}

\section{Optimization}

As assumed, no differences in $K^{\text {trans }}$ were found between days 14 and $21(p=0.80)$. Hence, the data of both days were pooled for further analyses. Table 3 shows the values for pharmacokinetic parameters estimated with various pharmacokinetic models and corresponding fit errors for the control tibialis muscle.

Between the two-parameter models, the Patlak model gave best fit for the MCA $(p<0.001$ for all animals). RFE were $5.1 \pm 0.4 \%$ (mean $\pm \mathrm{SE}$ ) and $13.0 \pm 0.9$ $\%$ for Patlak and GKM, respectively. For the SCA the RFEs of the GKM were smallest $(p<0.001$ in 5 out of 6 animals. RFE were $11.1 \pm 0.6 \%$ and $6.3 \pm 1.2$ $\%$ for Patlak and GKM, respectively. Expansion to the extended GKM gave a slight, yet significant improvement in fit quality for both the SCA $(p<0.001$ in 5 out of 6 animals; RFE $=4.8 \pm 0.4 \%$ ). For the MCA, the improvement was significant in 1 out of 6 rats $(p=0.04)$, whereas a deterioration was observed in 4 animals 
$(p<0.001)$

Table 5.3: Pharmacokinetic parameters estimated with various pharmacokinetic models and corresponding fit errors (mean \pm standard error) for the tibialis muscle in the control limb. * indicate the best fit error for the two-parameter models. Expansion to the three-parameter extended generalized kinetic model (extended GKM) gave a significant improvement of the fit error for the SCA.

\begin{tabular}{llcc}
\hline & & MCA & SCA \\
\cline { 3 - 4 } GKM & $K^{\text {trans }}\left(10^{-3} \mathrm{~min}^{-1}\right)$ & $6.9 \pm 0.3$ & $65.7 \pm 5.1$ \\
& $v_{\mathrm{e}}(\%)$ & $7.1 \pm 0.6$ & $12.1 \pm 0.5$ \\
& fit error $(\%)$ & $13.0 \pm 0.9$ & $6.3 \pm 1.2 *$ \\
& & \\
Patlak & $K^{\text {trans }}\left(10^{-3} \mathrm{~min}^{-1}\right)$ & $3.6 \pm 0.1$ & $24.4 \pm 0.9$ \\
& $v_{\mathrm{p}}(\%)$ & $0.9 \pm 0.1$ & $2.4 \pm 0.3$ \\
& fit error $(\%)$ & $5.1 \pm 0.4$ & $11.1 \pm 0.6$ \\
extended & & \\
GKM & $K^{\text {trans }}\left(10^{-3} \mathrm{~min}^{-1}\right)$ & $3.8 \pm 0.1$ & $50.4 \pm 3.2$ \\
& $v_{\mathrm{e}}(\%)$ & $45.2 \pm 9.4$ & $12.3 \pm 0.5$ \\
& $v_{\mathrm{p}}(\%)$ & $0.9 \pm 0.1$ & $1.1 \pm 0.3$ \\
fit error $(\%)$ & $5.2 \pm 0.4$ & $4.7 \pm 0.6$ \\
\hline
\end{tabular}

GKM: generalized kinetic model; MCA: medium-sized contrast agent; SCA: small contrast agent; $K^{\text {trans }}$ : transfer constant; $v_{\mathrm{e}}$ : volume fraction of the interstitium; $v_{\mathrm{p}}$ : volume fraction of the plasma.

Fitting with the extended GKM gave large estimation uncertainties (84\%) for $v_{\mathrm{e}}$ with the MCA (figure 5.2). Compared to the extended GKM, the simpler Patlak model had significantly lower estimation uncertainties for both $K^{\text {trans }}$ and $v_{\mathrm{e}}(p=$ 0.03 for both), as well as lower parameter interdependencies between $K^{\text {trans }}$ and $v_{p}$ $(p=0.03)$. For the SCA, estimation uncertainties of $K^{\text {trans }}$ and $v_{\mathrm{e}}$ did not differ between the normal and extended GKM. Interdependency between $K^{\text {trans }}$ and $v_{e}$ was stronger for the GKM $(p=0.03)$. However, the estimation uncertainty of $v_{p}$ with the extended GKM for the SCA was very large (>1000\%), and it was accompanied by a strong interdependency between $v_{p}$ and $K^{\text {trans }}$, which might negatively influence $K^{\text {trans }}$ estimation.

Although expansion to the extended GKM resulted in a significantly better fit for the SCA, it was accompanied by introduction of parameter $v_{p}$ that could not be reliably estimated and had strong parameter interdependency with $K^{\text {trans }}$. As the improvement in fit error was small, we selected the GKM as the optimal for the SCA. For the MCA, the Patlak was the best model in terms of fit error, parameter uncertainty and error interdependency and was therefore considered the optimal model. 

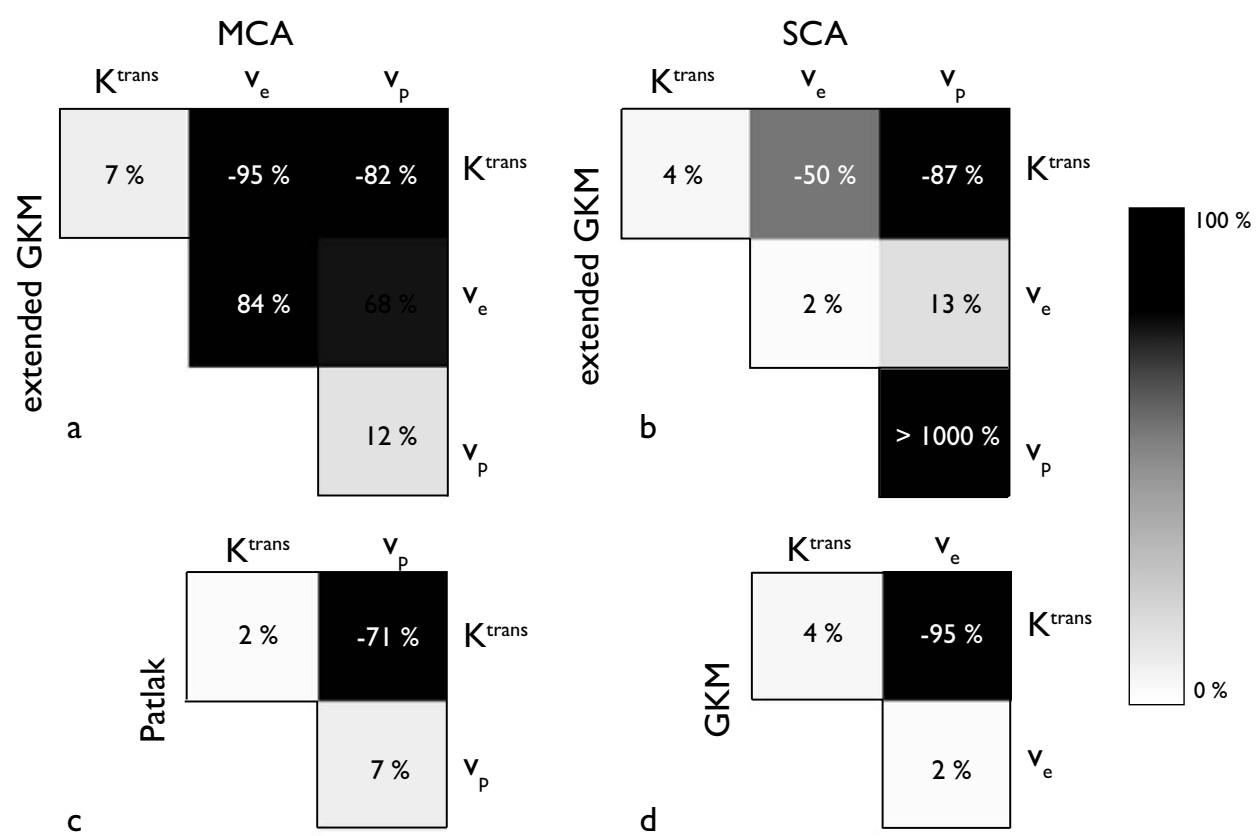

Figure 5.2: Averaged elements of the covariance matrix for fits with the extended generalized kinetic model (upper panels) and the optimal two-parameter models (MCA: Patlak, panel C; SCA: generalized kinetic model (GKM), panel d) for the control tibialis anterior muscle. Diagonal elements represent the percentage parameter estimation uncertainties and off-diagonal elements represent parameter interdependencies. A positive interdependency between two parameters indicates that underestimation of one parameter is likely to be accompanied by underestimation in the other, while a negative interdependency tells that underestimation in one parameter is often accompanied by overestimation of the other (and vice versa). For a high interdependency the chance of the occurrence of this effect is high.

\section{Detection of microvascular differences}

Optimal model: Both MCA and SCA were successful in detecting microvascular differences using $K^{\text {trans }}$ estimated with the optimal model (figure 5.3). For the MCA, $K^{\text {trans }}$ was $4.8 \pm 0.210^{-3} \mathrm{~min}^{-1}$ (mean \pm standard error) and $3.6 \pm 0.110^{-3} \mathrm{~min}^{-1}$ for the red soleus and white tibialis muscle, respectively, $p<0.01$. With the SCA, $K^{\text {trans }}$ was $81 \pm 510^{-3} \mathrm{~min}^{-1}$ (soleus) and $66 \pm 510^{-3} \mathrm{~min}^{-1}$ (tibialis) $(p<0.01$ ). $K^{\text {trans }}$ was significantly lower in the ischemic limb compared to the control limb for both soleus and tibialis $(p<0.02)$. These differences ranged from $5-20 \%$ and were similar for both MCA and SCA. Correlation of $K^{\text {trans }}$ between the two contrast agents was high (Pearson $R=0.81 ; p<0.01$ ). 

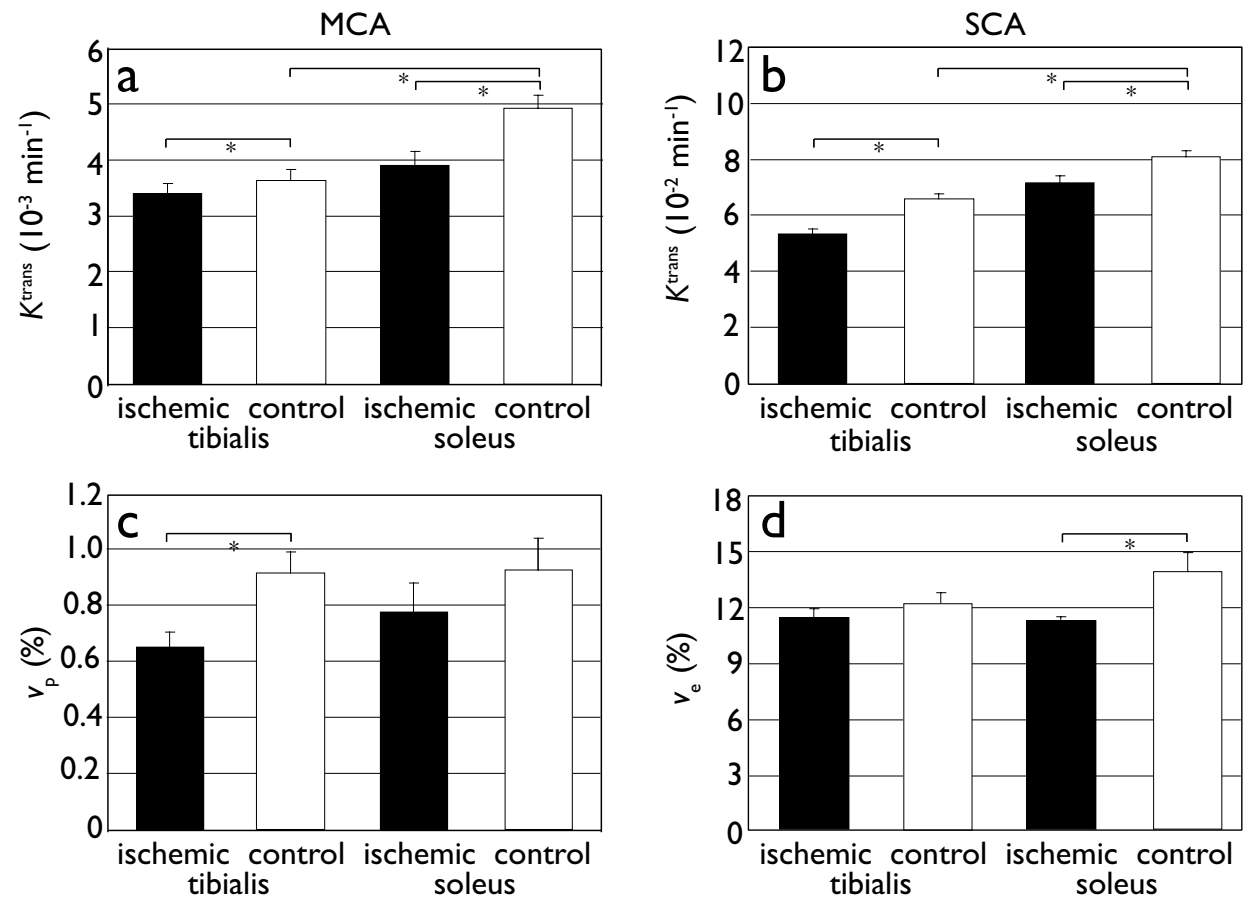

Figure 5.3: Pharmacokinetic parameters of the different muscle groups as estimated with the optimal models. With the MCA, pharmacokinetic modeling gave estimations for $K^{\text {trans }}$ (panel a) and $v_{p}$ (panel $c$ ), and with the SCA $K^{\text {trans }}$ (panel b) and $v_{e}$ (panel d) were estimated. Error bars indicate the standard error of the mean. Significant differences $(p<0.05)$ between muscle groups were indicated with *. 
Extended GKM: With the extended GKM the same trends were observed compared to the optimal model (figure 5.4). However, for the MCA only the difference in $K^{\text {trans }}$ between ischemic and control soleus, and between control soleus and tibialis were significant ( $p=0.03$ and 0.01 , respectively). Moreover, for the SCA only the difference between ischemic and control tibialis nearly reached significance $(p=$ $0.06)$, whereas neither the differences in $K^{\text {trans }}$ between ischemic and control soleus $(p=0.69)$ nor between red and white muscle groups were significant $(p=0.22)$. With the MCA, unrealistically high values for $v_{e}$ were obtained $\left(v_{e}>70 \%\right)$. For the SCA, $v_{p}$ was significantly higher in the control soleus compared to both control tibialis and ischemic soleus ( $p=0.01$ for both).

\section{Model-free approach}

\section{Optimization}

With the MCA, the highest sensitivity for microvascular differences was found for $T_{\text {AUC }}=12 \mathrm{~min}$. For the SCA, the difference in AUC between the various muscle groups was largest for $T_{A U C}=3 \mathrm{~min}$. Using these $T_{A \cup C}$, model-free parameter $A U C$ was equally effective in detecting microvascular differences as $K^{\text {trans }}$ (figure 5.5). For both contrast agents, AUC was significantly decreased in the muscle groups in the ischemic limb compared to the control limb $(p<0.03)$. In the control limb, the soleus had a significantly larger AUC than the tibialis muscle $(p<0.01)$.

\section{Comparison with model-based parameters}

The contribution of pharmacokinetic properties, as estimated with the optimal model for each contrast agent, to model-free parameter AUC strongly depended on $T_{A U C}$ (figure 5.6). For the MCA, a longer $T_{A \cup C}$ resulted in a stronger contribution of $K^{\text {trans }}$ and a weaker influence of $v_{p}$. In contrast, for the SCA the contribution of $K^{\text {trans }}$ decreased with increasing $T_{A \cup C}$, whereas ve increasingly contributed.

\section{Image quality}

Relative maximal signal enhancement in the control tibialis did not differ between contrast agents $\left(E_{\max }=23.5 \pm 0.5 \%\right.$ (mean \pm SE) and $24.3 \pm 2.0 \%$ for the MCA and SCA, respectively, $p=0.45)$. Noise level on $C_{t}$ was equal for both contrast agents (MCA: $3.5 \pm 0.4 \%$; SCA: $3.2 \pm 0.3 \% ; p=0.54$ ). Pre-contrast SNR was higher for the SCA ( $45 \pm 10$ for the MCA images and $69 \pm 18$ for the SCA images, $p=0.015)$, which is due to the larger acquired voxel size. The higher spatial resolution for the MCA facilitated more accurate delineation of the various muscle 

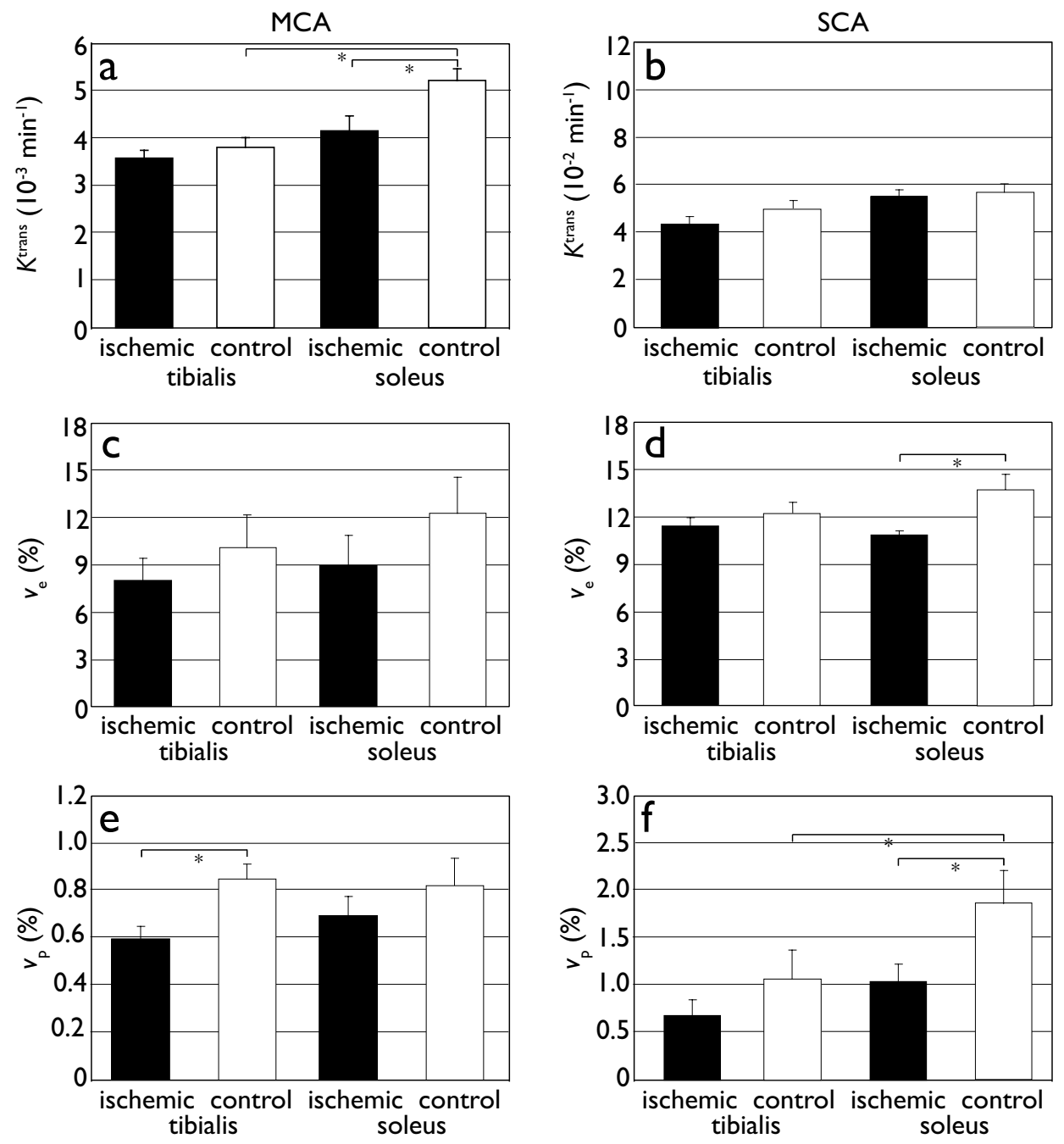

Figure 5.4: Pharmacokinetic parameters $K^{\text {trans }}$ (panels a and b), $v_{e}$ (panels $c$ and $d$ ), and $v_{p}$ (panels e and $f$ ) of the different muscle groups as estimated with the extended generalized kinetic model. Error bars indicate the standard error of the mean. Significant differences $(p<0.05)$ between muscle groups were indicated with *.

groups and better exclusion of non-muscular tissues such as bone and large blood vessels (figure 5.7). 

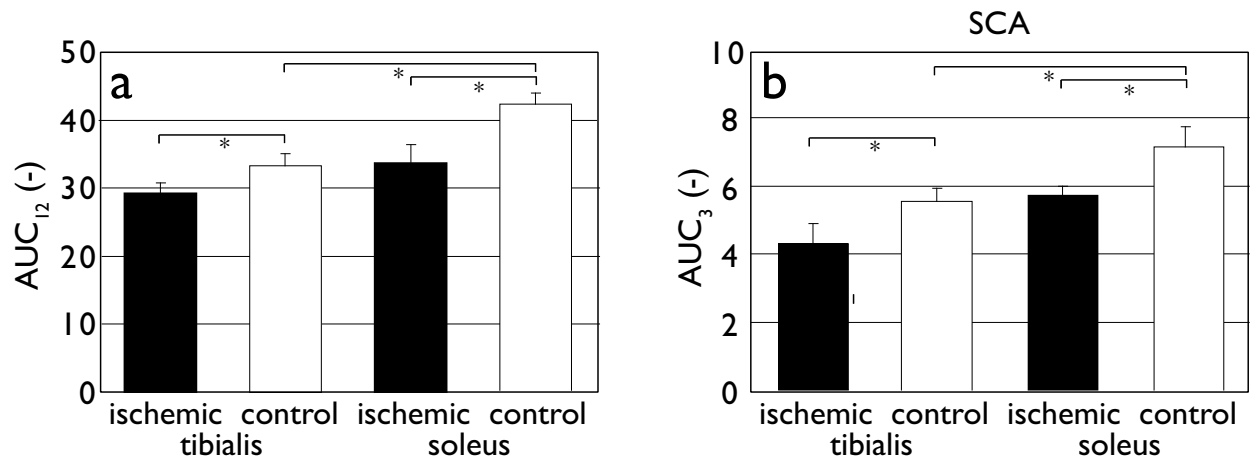

Figure 5.5: AUC values in the different muscle groups for the MCA (panel a) and the SCA (panel b). For each contrast agent the AUC that corresponded best with $K^{\text {trans }}$ was chosen (SCA: $T_{\text {AUC }}=3 \mathrm{~min}$; MCA: $T_{\text {AUC }}=12 \mathrm{~min}$ ). Error bars indicate the standard error of the mean. Significant differences $(p<0.05)$ are indicated with *.
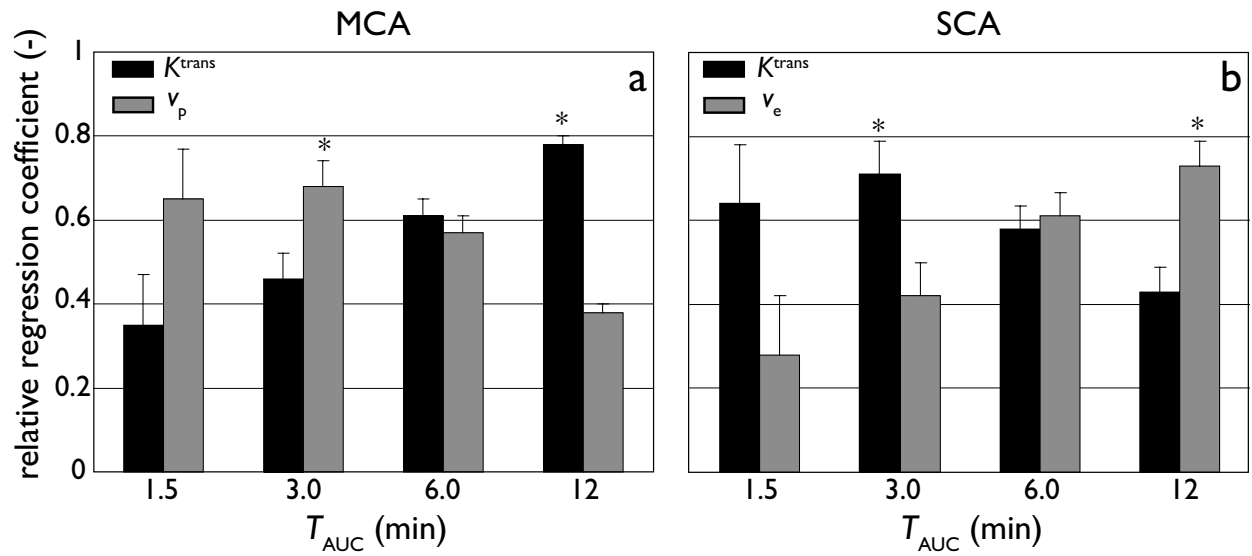

Figure 5.6: Standardized regression coefficients in multivariate linear regression between AUC at $T_{\mathrm{AUC}}=1.5,3,6$, and 12 minutes after contrast injection, and pharmacokinetic parameters estimated with the optimal model. For the MCA, the contribution of $K^{\text {trans }}$ increased with increasing $T_{\text {AUC }}$, while the influence of $v_{p}$ decreased. Alternatively, for the SCA the contribution of $K^{\text {trans }}$ decreased with increasing $T_{A \cup C}$, whereas $v_{e}$ increasingly contributed. Error bars indicate the standard error of the mean. Parameters that contributed significantly most $(p<0.05)$ are marked with *. 


\subsection{Discussion}

\section{Current findings}

In this study the potential of an MCA in detecting skeletal muscle microvascular differences in a rabbit hind limb ischemia model was investigated and compared to an SCA. It was shown for the first time that contrast agent specific data analysis resulted in a higher sensitivity of model-based parameter $K^{\text {trans }}$ and model-free parameter AUC for microvascular differences. With optimized data analysis strategies, physiological and pathophysiological microvascular differences could be detected with both contrast agents and model-based and model-free analysis. The MCA was equally successful in detecting microvascular differences as the SCA, at a higher spatial resolution.

\section{Optimization of model-based approach}

Theoretically, quantification of pharmacokinetic parameters requires fast acquisition protocols for small contrast agents [88], whereas a long acquisition time should be used for larger contrast agents [112]. However, in clinical practice these requirements are not easily met due to conflicting demands on patient compliance, spatial resolution and coverage of the anatomy of interest. Selection of the appropriate model to fit these imperfect DCE-MRI data is not straightforward.

For SCAs, filling of the vascular space and extravasation occurred almost concurrently, which resulted in a high estimation uncertainty on $v_{p}$ and a strong interdependency between $K^{\text {trans }}$ and $v_{p}$. It is therefore not recommended to include $v_{p}$ in the analysis. On the contrary, MCAs act as intravascular agents during the first-pass [137]. Consequently, bolus arrival and extravasation appeared as clearly distinctive features in the tissue concentration curves. As a result, estimation uncertainties on $v_{p}$ and $K^{\text {trans }}$ were relatively small. However, the MCA tissue curve did not reach its maximum within the duration of the experiment. An incomplete washout measurement resulted in poor performance of the extended GKM. Despite the extra parameter, fit errors were larger in the extended GKM compared to the Patlak model, which was the result of forcing the additional parameter $v_{\mathrm{e}}$ to remain within the natural boundaries of 0 and $100 \%$ during the fitting procedure. Estimation uncertainties on $v_{e}$ were large, which negatively influenced the uncertainty on $K^{\text {trans }}$, as a consequence of the larger error interdependencies between $K^{\text {trans }}$ and $v_{e}$. Reliable estimation of $v_{e}$ can be obtained by elongation of the total acquisition time $[112,156]$. However, in most studies the parameter of interest is $K^{\text {trans }}$, which can be reliably estimated within clinically acceptable acquisition times ( $<10 \mathrm{~min})$ by using the Patlak model.

Our work underscores the importance of model selection in DCE-MRI data anal- 
ysis. Because of parameter interdependency, the use of a model including parameters which cannot be estimated reliably may have a detrimental effect on the estimation of the other parameters. Which model is optimal depends on the specific features present in the concentration time curves. As this varies with acquisition settings (especially dynamic and total acquisition time), contrast agent size and microcirculatory properties of the tissue of interest, so should the choice of the pharmacokinetic model. Analysis of the quality of fit, which has been used in similar studies $[150,156]$ may be the most straightforward to implement as quality control, but we feel that estimation uncertainties and parameter interdependencies should also be used when assessing the validity of a model.

\section{Comparison between model-free and model-based parame- ters}

Model-free parameter extraction has several advantages over model-based parameter estimation. It is more robust and obviates the need for an arterial input function, which is a major source of error in pharmacokinetic modeling [15]. However, the comparison of patients becomes more difficult, as the relationship between AUC and microvascular properties depends on the injection protocol and bolus shape [158]. The results of this study revealed that the degree to which pharmacokinetic properties are reflected in the AUC, and consequently the physiological meaning, depends on $T_{\text {AUC }}$. Moreover, the sensitivity for microvascular differences appeared to be highest when the contribution of $K^{\text {trans }}$ was relatively largest. The pharmacokinetic properties of the contrast agent should therefore also be taken into account when selecting $T_{\text {AUC }}$.

\section{Detection of microvascular differences}

This study showed that the highest sensitivity for changes in $K^{\text {trans }}$ was obtained when data analysis strategies were adapted to the pharmacokinetic properties of the contrast agent. A fair comparative study requires optimal data analysis for each contrast agent. Using a unified analysis might therefore not maximally benefit from the advantages of each contrast agent, or introduce a bias. We therefore recommend that both model-free and model-based analysis strategies are tailored to the characteristics of each individual contrast agent.

The physiological interpretation of $K^{\text {trans }}$ is not straightforward, as it is related to both flow $(F)$ and permeability surface area product $(P S)$ by $K^{\text {trans }}=F \cdot\left(1-e^{\frac{-P S}{F}}\right)$ [83]. The degree to which $F$ and $P S$ contribute is determined by their ratio. If $P S>>F$, as is the case for SCAs, contrast agent extravasation is considered to be flow-limited and $K^{\text {trans }}$ is mainly a measure for microvascular flow. For $P S<<F$ , $K^{\text {trans }}$ is predominantly determined by $P S$ [83]. The low permeability of MCAs 

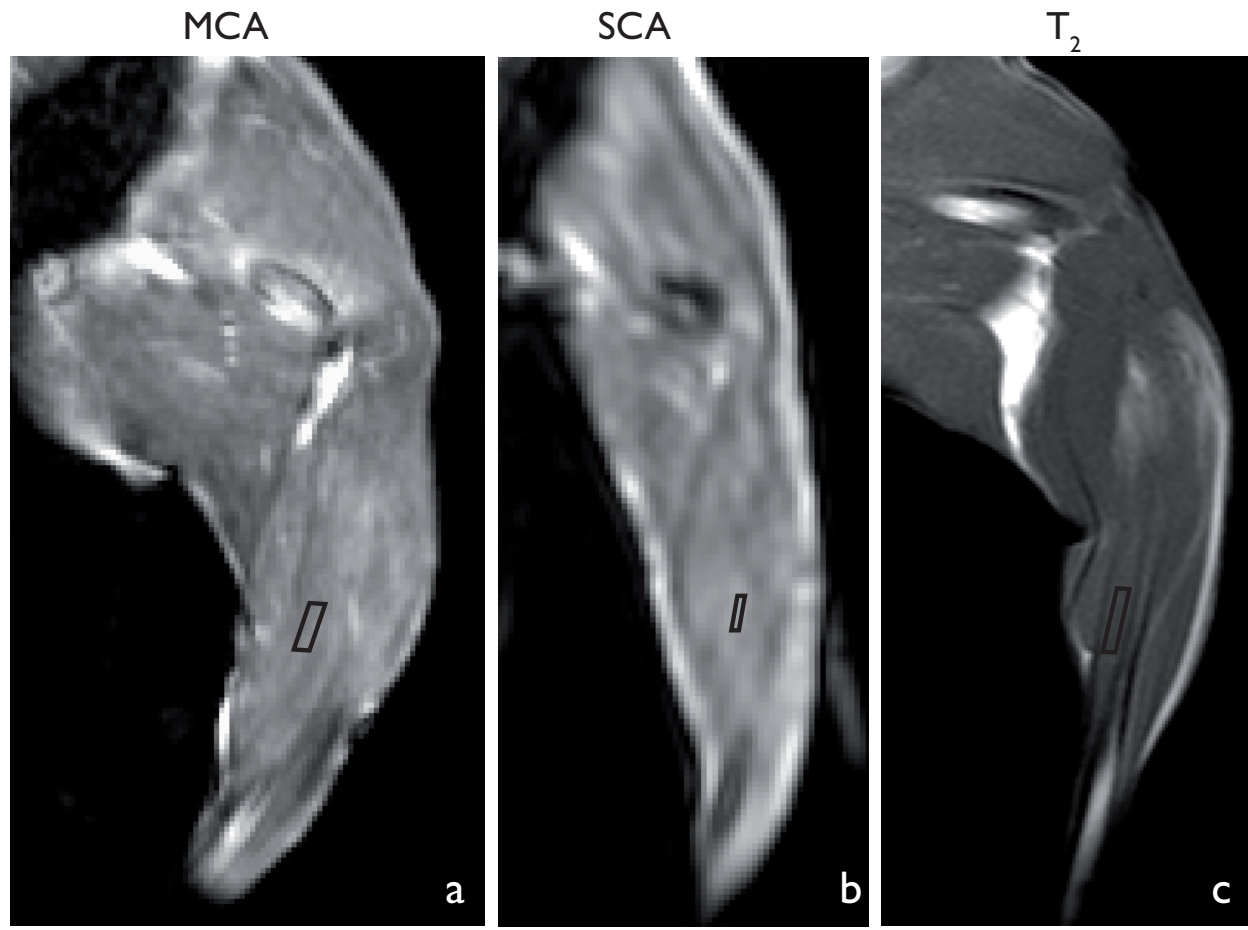

Figure 5.7: MR images obtained with the DCE-MRI protocol for the MCA (panel a) and SCA (panel b). Voxel size was $0.8 \times 0.7 \times 1.3 \mathrm{~mm}^{3}$ for the MCA and $2.0 \times 1.6 \times 2.5 \mathrm{~mm}^{3}$ for the SCA. ROIs delineated in black indicate the soleus. As a reference a high resolution $T_{2}$-weighted image with voxel size $0.5 \times 0.5 \times 4.0 \mathrm{~mm}^{3}$ is shown (panel c). 
therefore is an advantage in cancer imaging $[92,93]$ where hyperpermeable vessels are a key feature of tumor growth [159], but might be a disadvantage for perfusion studies. Strong tissue perfusion is generally achieved by a high number of opened capillaries. As DCE-MRI is a functional technique, the capillary surface area $S$ represented in $K^{\text {trans }}$ is the surface area of the actually perfused capillaries, rather than the total capillary surface area. The high correlation between $K^{\text {trans }}$ of the MCA and the SCA found in this study suggests that microvascular flow and surface area are indeed highly related characteristics in skeletal muscle tissue, making $K^{\text {trans }}$ of an MCA a suitable parameter to assess perfusion differences with either contrast agent.

Using our contrast agent specific data analysis methods, physiological microvascular differences between muscle groups with different muscle fiber compositions could be detected. $K^{\text {trans }}$ was $20-25 \%$ higher in the soleus muscle, which primarily contains highly perfused "slow-twitch" type I fibers [56], compared to the tibialis anterior muscle, with mainly of "fast-twitch" type II fibers. Our findings compare reasonably with the $35 \%$ difference found by Thompson et al. [78] with an SCA in humans in a reactive hyperemia experiment, and by Faranesh et al. [77] with an MCA in rabbits without any vasodilation.

In this study, we found that $K^{\text {trans }}$ was still decreased in the calf muscles of the ligated limb two to three weeks after ligation, which corresponded well with the results from a previous study [76]. Changes in capillary density due to angiogenesis and restoration of rest blood flow were found to normalize after 10 days post ligation. However, reserve blood flow has been demonstrated to still be impaired [32]. In our experiments, hyperperfusion was induced by administration of adenosine, thereby increasing perfusion differences in normal and ischemic calf muscles. We therefore believe that differences in $K^{\text {trans }}$ found represented perfusion differences.

\section{Limitations}

In this study a standardized arterial input function was used for each animal and each limb. This input function was obtained by taking blood samples from the femoral artery. The actual input might be different for the ischemic and control muscle tissue of the lower limb as the upstream macrovasculature adapts after ligation. The actual input function of the ischemic muscle tissue is expectedly delayed and more dispersed compared to the control limb due to the presence of collateral arteries which delay contrast agent delivery [76]. Due to limited spatial resolution it was not feasible to determine input functions for each animal and each limb. We believe that our standard input function with correction for arrival time best approximates the concentration time course in the hind limb. By not correcting for differences in the arterial input function, $K^{\text {trans }}$ may become a combination of tissue perfusion and impaired collateral blood supply. As our standard AIF does not incorporate fast varying components of the first passage, which are most likely to be affected by the collateral bed, we expect the influence on the model selection to be small. 
In this study, the measured perfusion differences were not validated by microsphere perfusion measurements. Other studies have already demonstrated the strong correlation between DCE-MRI related parameters and muscle tissue perfusion as measured with microspheres $[85,86,160]$. The focus of our study was therefore on improving the reliable estimation of these parameters for differently sized contrast agents. Microsphere experiments do not provide such information.

\section{MCAs in DCE-MRI}

The use of MCAs in DCE-MRI has great clinical potential over SCAs. The main advantage is their less stringent requirements on dynamic acquisition times, which can be exploited to increase spatial resolution or coverage. Improved spatial resolution facilitates delineation of muscle groups and makes it easier to exclude non-muscular tissue such as large blood vessels and bone. The smaller voxel size may lead to a lower SNR, as was seen in this study. However, when the analysis is ROI-wise rather than voxel-wise, the noise on the tissue concentration time curve is more relevant than SNR, and in this study the noise level appeared to be equal for both contrast agents.

Gadomer is a relatively large MCA. Other MCAs, such as P972 (molecular weight: $6.5 \mathrm{kDa}$ [161]) and P846 (molecular weight: 3.5 [162]) are considerably smaller, which is likely to affect the choice of pharmacokinetic model. Which model is most appropriate depends on acquisition settings and tissue of interest as well as contrast agent size. The method proposed in this study can be used to define the best model in each situation.

Unfortunately, none of the MCAs is currently approved for use in humans. The value of only approved gadolinium-based intravascular contrast agent, gadofosveset trisodium, to DCE-MRI is limited. Gadofosveset itself has a molecular weight of $1.0 \mathrm{kDa}$, but after non-covalently binding to albumin, the molecular weight becomes $70 \mathrm{kDa}$. In human plasma, 80 - $96 \%$ of the contrast agent is bound [163], yet during the first passage of the contrast agent, a large fraction ( $74 \%$ [104]) is still unbound and extravasates more rapidly. The variation in fraction of bound contrast agent make pharmacokinetic modeling complicated, resulting in a poor correlation with histology [164].

The greatest benefit of MCAs in cardiovascular applications may be gained in cardiac DCE-MRI, as demonstrated by Gerber et al. [165] in pigs. Here, temporal requirements are even stricter and the need for high spatial resolution is more urgent, because detailed information of the spatial extent (transmurality) of the infarcted area is an important predictor of recovery [166]. 
Chapter 5. Contrast agent size in DCE-MRI of muscle perfusion

\section{Conclusion}

For optimal estimation of microvascular parameters, both model-based and modelfree analysis should be adapted to the pharmacokinetic properties of the contrast agent, as the detection of microvascular differences is most sensitive when an analysis strategy tailored for each contrast agent is used. Pathophysiological and physiological microvascular differences between muscle groups could be detected with model-based parameter $K^{\text {trans }}$ and model-free parameter AUC. The MCA was equally successful as the SCA, at a higher spatial resolution and comparable SNR. 




\section{Morphological and functional monitoring of therapeutically induced neovascularization in ischemia with MRI}




\section{Abstract}

Therapies that stimulate arteriogenesis or angiogenesis may serve as an alternative to invasive treatment strategies to relieve myocardial and skeletal muscle ischemia. The purpose of this study was to assess the potential of comprehensive functional and morphological MRI techniques for non-invasive in vivo evaluation of neovascularization therapies.

Rats $(\mathrm{N}=32)$ underwent unilateral femoral artery ligation. Angiogenesis and angiogenesis-related hyperpermeability were promoted by vascular endothelial growth factor (VEGF), and arteriogenesis by monocyte chemoattractant protein (MCP-1). Combined angiogenesis and arteriogenesis without hyperpermeability were created by a combination of fibroblast growth factor (FGF) and platelet-derived growth factor (PDGF). The control group received phosphate buffer solution (PBS). All agents were administered intra-arterially by osmotic minipump.

On days 2, 7 and 21 post ligation, the animals underwent an MRI exam consisting of time-of-flight MR angiography (TOF-MRA) to evaluate arteriogenesis by visualizing the collateral arteries, $T_{2}$-weighted imaging to detect edema (i.e. hyperpermeability) associated with angiogenesis, and dynamic contrast-enhanced (DCE) MRI to assess muscle perfusion.

On TOF-MR angiograms, collateral formation appeared enhanced by the combination of FGF and PDGF and by MCP-1. $T_{2}$-weighted images showed prolonged edema in animals with stimulated angiogenesis. Functional assessment with DCEMRI demonstrated lower extravasation and local plasma clearance rates in the ischemic compared to the control limb. All treatment groups showed an accelerated improvement in tissue perfusion with respect to the control group.

In conclusion, MRI allows comprehensive, non-invasive characterization and monitoring of the arteriogenic and angiogenic responses to neovascularization therapy and their effects on tissue perfusion, by demonstrating time- and treatment-dependent differences between ischemic and control muscle.

Submitted 


\subsection{Introduction}

Peripheral and coronary artery disease (PAD and CAD, respectively) are clinical expressions of atherosclerosis characterized by stenosis or occlusion of the arteries supplying the extremities or myocardium, respectively. In a relatively large group of patients, conventional invasive treatment strategies such as percutaneous transluminal angioplasty (PTA) and surgery are not an option or are unsuccessful. This results in a high morbidity and mortality $[3,4,167]$. As the disorders are associated with insufficient collateralization and decreased microvascular density $[5,168]$, therapies that promote arteriogenesis (i.e. maturation of arterioles into functional collateral arteries) and angiogenesis (i.e. sprouting of capillaries from preexisting vessels) seem attractive as an alternative or adjuvant treatment.

Stimulation of neovascularization can be achieved by administration of cytokines that promote the formation and development of blood vessels. Extensive research has identified many of these activators (for an overview, see $[10,11,25]$ ). Some factors, such as vascular endothelial growth factor (VEGF $[12,13]$ ) mainly stimulate angiogenesis, while others are primarily associated with arteriogenesis, such as monocyte chemoattractant protein-1 (MCP-1 [24]). Many growth factors play a role in both forms of neovascularization. Examples from this group are fibroblast growth factor (FGF-) 2 and platelet-derived growth factor (PDGF-) BB, which exhibit synergism when administered together [27]. Animal studies have shown the potential of growth factor therapy in animal hindlimb ischemia models $[94,169,170]$.

Clinical trials with FGF-2 have shown promising long-term results regarding therapydependent improvements in peak walking time or exercise tolerance (measured 90 days after the start of treatment) and amputation-free survival [35-37]. Thus far, it remains unknown how functional responses in patients relate to vascular changes. Further development and clinical introduction would therefore greatly benefit from the availability and development of imaging techniques that can monitor therapeutic efficacy in an earlier stage of therapy [39], which may enable individualized treatment strategies. Magnetic resonance imaging (MRI) is a safe and non-invasive imaging modality that does not use ionizing radiation. It can therefore be used repeatedly without imposing a risk on the patient. Due to its versatility, MRI can be used to obtain both anatomical and functional information within a single session.

The arteriogenic response can be assessed morphologically with MR angiography (MRA). This technique has already been widely introduced as a diagnostic tool for patients with PAD and CAD $[42,46]$, and studies in both animals and patients have demonstrated that collateral arteries could be visualized $[97,98,120]$. VEGF-induced angiogenesis is associated with an increase in vascular permeability [10], which can cause edema $[171,172]$. T2-weighted MR imaging is an effective and straightforward way to estimate muscle water content and to visualize edematous myocardium [173, 174] and skeletal muscle tissue [175]. The therapeutic effect on tissue perfusion can be assessed with dynamic contrast-enhanced (DCE) MRI. This technique, which 
is based on the extravasation of a contrast agent, provides information on muscle microvasculature $[76,77,86]$, as DCE-MRI-related parameters correlate well with tissue perfusion measured with microspheres [85, 86].

The purpose of this study was to assess the potential of combined functional and morphological MR techniques for the in vivo evaluation of therapeutically induced neovascularization. To this end, MRI-derived measures reflecting arteriogenesis, angiogenesis, angiogenesis related hyperpermeability and their effects on muscle perfusion were determined at multiple time points in a rat ischemic hindlimb model in which either or both forms of neovascularization in presence or absence of hyperpermeability are stimulated with growth factors. 


\subsection{Methods}

\section{Animal model}

This study was approved by the local animal ethics committee. 32 male SpragueDawley rats ( $N=8$ per treatment group, pre-operative weight 300 - $350 \mathrm{~g}$ ) underwent unilateral femoral artery ligation. For continuous administration of growth factors, a catheter connected to an osmotic minipump (Alzet model 2ML4, Durect Corp) was inserted just above the bifurcation of the popliteal and saphenous artery, with the tip of the catheter placed just below the origin of the circumflex femoral artery, thus creating a ligation.

Animals were randomly assigned into four groups. In three treatment groups, either or both forms of neovascularization were stimulated with growth factors whose effects on neovascularization are well known. The first group received VEGF-A, which stimulates angiogenesis and causes hyperpermeability. To the second group, MCP-1, a potent arteriogenic growth factor, was administered. The third group received a combination of FGF-2 and PDGF-BB (henceforth called the FGF group), which promotes both forms of neovascularization in the absence of hyperpermeability. A fourth group receiving a phosphate buffer solution (PBS) served as a control. The growth factors (PeproTech Inc) and PBS were administered at a rate of 2.5 $\mu \mathrm{L} /$ hour, maintained during the complete course of the experiment. The growth factor concentration was $28 \mu \mathrm{g} / \mathrm{mL}$ diluted in PBS for all growth factors. After surgery, the animals were examined daily for necrosis or abnormal limb swelling.

For the surgery and subsequent MRI measurements, anesthesia was induced by placing the animal in an induction box filled with a mixture of oxygen and isoflurane. During both the intervention and MR exams, the rats were ventilated with $3 \%$ isoflurane in oxygen. Analgesia was provided by intramuscular injection of buprenorphine $(0.01 \mathrm{mg} / \mathrm{kg})$ immediately before the start of surgery. A cannula was placed in the rats' tail vein to enable contrast agent injection. After the final MRI session at day 21 , the animals were sacrificed by an intravenous lethal injection of pentobarbital. Subsequently, the calf muscles were exposed and visually inspected for muscle damage.

\section{MRI protocol}

On days 2, 7, and 21 post surgery, the animals were imaged in supine position in a 7.0 Tesla MR system with a birdcage quadrature coil (Bruker Biospin, Ettlingen, Germany). The MR protocol consisted of time-of-flight MR angiography (TOFMRA), $T_{2}$-weighted anatomic images, and DCE-MRI. 


\section{TOF-MRA}

A 2D flow-compensated gradient echo sequence was used to visualize the collateral arteries in the upper limb. 150 axial slices with matrix size $200 \times 256$ were acquired. Voxel size was $0.49 \times 0.49 \times 0.55 \mathrm{~mm}^{3}$. TR/TE/FA were $28 \mathrm{~ms} / 4.5 \mathrm{~ms} / 76^{\circ}$, and number of signal averages was 2 . A flow saturation slab located distally to the imaging plane was applied to suppress venous enhancement. Total acquisition time was $35 \mathrm{~min}$.

\section{$T_{2}$-weighted imaging}

A 2D $T_{2}$-weighted turbo spin echo sequence (turbo factor 8 ) was used to measure calf muscle edema. TR/TE/FA were $4000 \mathrm{~ms} / 35 \mathrm{~ms} / 90^{\circ}$. Matrix size was $256 \times 256$ and number of coronal slices was 30 . Acquired voxel size was $0.23 \times 0.23 \times 1.7 \mathrm{~mm}^{3}$.

\section{DCE-MRI}

For perfusion imaging, a dynamic $T_{1}$-weighted $3 \mathrm{D}$ gradient echo sequence was used. TR/TE/FA were $6.9 \mathrm{~ms} / 2.5 \mathrm{~ms} / 25^{\circ}$. Matrix and voxel sizes were $128 \times 128 \times 30$ and $0.47 \times 0.47 \times 1.7 \mathrm{~mm}^{3}$, respectively. Number of dynamic phases was 65 and total acquisition time was $22 \mathrm{~min}$. During acquisition, $0.1 \mathrm{mmol} / \mathrm{kg}$ body weight Gadomer (Bayer Schering Pharma AG, Berlin, Germany) was administered manually after 1.5 minutes, followed by a $0.5 \mathrm{~mL}$ saline flush. The concentration of the solution was diluted to $0.1 \mathrm{M}$ (original vial concentration $0.5 \mathrm{M}$ ) and the injected volume was 1 $\mathrm{mL} / \mathrm{kg}$ body weight. The injection rate was $0.05 \mathrm{~mL} / \mathrm{s}$.

\section{Image analysis}

\section{TOF-MRA}

The arteriogenic response was assessed by quantifying the fraction of pixels representing collateral arteries and feeding branches in the area of collateral formation on maximum intensity projections (MIPs) in the cranio-caudal direction. The stack of axial slices over which the MIPs were computed was determined for each set separately based on vascular anatomy. The most proximal slice was chosen just above the origin of the circumflex femoral artery and the most distal slice was positioned above the bifurcation of the cranial and caudal tibial artery (see figure 6.1a). The MIPs were filtered with a 2D multi-scale vessel enhancement filter described by Kagadis et al. [126] to suppress noise and enhance vascular (i.e. line-shaped) structures. In the original MIPs, ROls were drawn in both the control and ligated limb (see figure $6.1 \mathrm{~b}$ ). The ROIs encompassed the femoral artery (in the control limb), the circum- 
flex femoral artery and the anterior branches of the internal iliac artery, and excluded the testicular artery, the iliac artery and its posterior branches.

In the ROI in the control limb, the signal intensities at which 80 and $95 \%$ of the pixels have lower signal intensity were determined ( $\mathrm{SI}_{80}$ and $\mathrm{SI}_{95}$, respectively). Segmentation based on signal intensities (SI) showed that in the ligated limb, pixels with $\mathrm{SI}$ between $\mathrm{SI}_{80}$ and $\mathrm{SI}_{95}$ were highly associated with collateral arteries (figure 3.1c). Pixels with SI higher than $\mathrm{SI}_{95}$ were found to represent branches of the femoral artery that feed the collateral arteries (figure 3.1d). The percentage of pixels between $\mathrm{SI}_{80}$ and $\mathrm{SI}_{95}\left(\mathrm{P}_{\text {small }}\right)$, and higher than $\mathrm{SI}_{95}\left(\mathrm{P}_{\text {large }}\right)$ were therefore used as a measure for the volume of collateral arteries and feeding branches, respectively. In the ROI in the control limb, $\mathrm{P}_{\text {small }}$ was $15 \%$ and $\mathrm{P}_{\text {large }}$ was $5 \%$ by definition.

\section{$T_{2}$-weighted imaging}

The degree of edema was measured on the $T_{2}$-weighted images. Signal intensity (SI) was measured in regions-of-interest (ROIs) drawn in the ischemic and control calf muscle. The degree of edema was expressed as the lateralization index $\left(\mathrm{LI}_{x}\right)$ between the control and ischemic limb:

$$
L I_{x}=\frac{S I_{\text {ischemic }}-S I_{\text {control }}}{S I_{\text {ischemic }}-S I_{\text {control }}}
$$

\section{DCE-MRI}

The ROIs used for $T_{2}$-weighted imaging were rescaled in MATLAB (The MathWorks, Natick, MA) to match the DCE data. The signal intensity time courses $S(t)$ in the ROIs were normalized according to:

$$
S_{n}(\mathrm{t})=\frac{S(\mathrm{t})-S_{0}}{S_{\max }-S_{0}}
$$

where $S_{0}$ is the pre-contrast signal intensity, averaged over four pre-contrast images and $S_{\max }$ the maximum signal intensity. $S_{n}(t)$ represents the time course of relative signal change and served as a surrogate measure for the tissue concentration time course $C_{t}(\mathrm{t})$.

The time series data were fitted with the pharmacokinetic model described by Tofts et al. [83]:

$$
C_{\mathrm{t}}(t)=K^{\mathrm{trans}} \int_{0}^{t} C_{\mathrm{p}}(\mathrm{u}) \mathrm{e}^{-k_{\mathrm{ep}}(t-\mathrm{u})} \mathrm{du}
$$

where $K^{\text {trans }}$ is the extravasation rate, $k_{\mathrm{ep}}$ the reflux rate, and $C_{\mathrm{p}}(\mathrm{t})$ the plasma concentration time course. $K^{\text {trans }}$ and $k_{\text {ep }}$ are related according to $k_{\mathrm{ep}}=\frac{K^{\text {trans }}}{V_{\mathrm{e}}}$, 

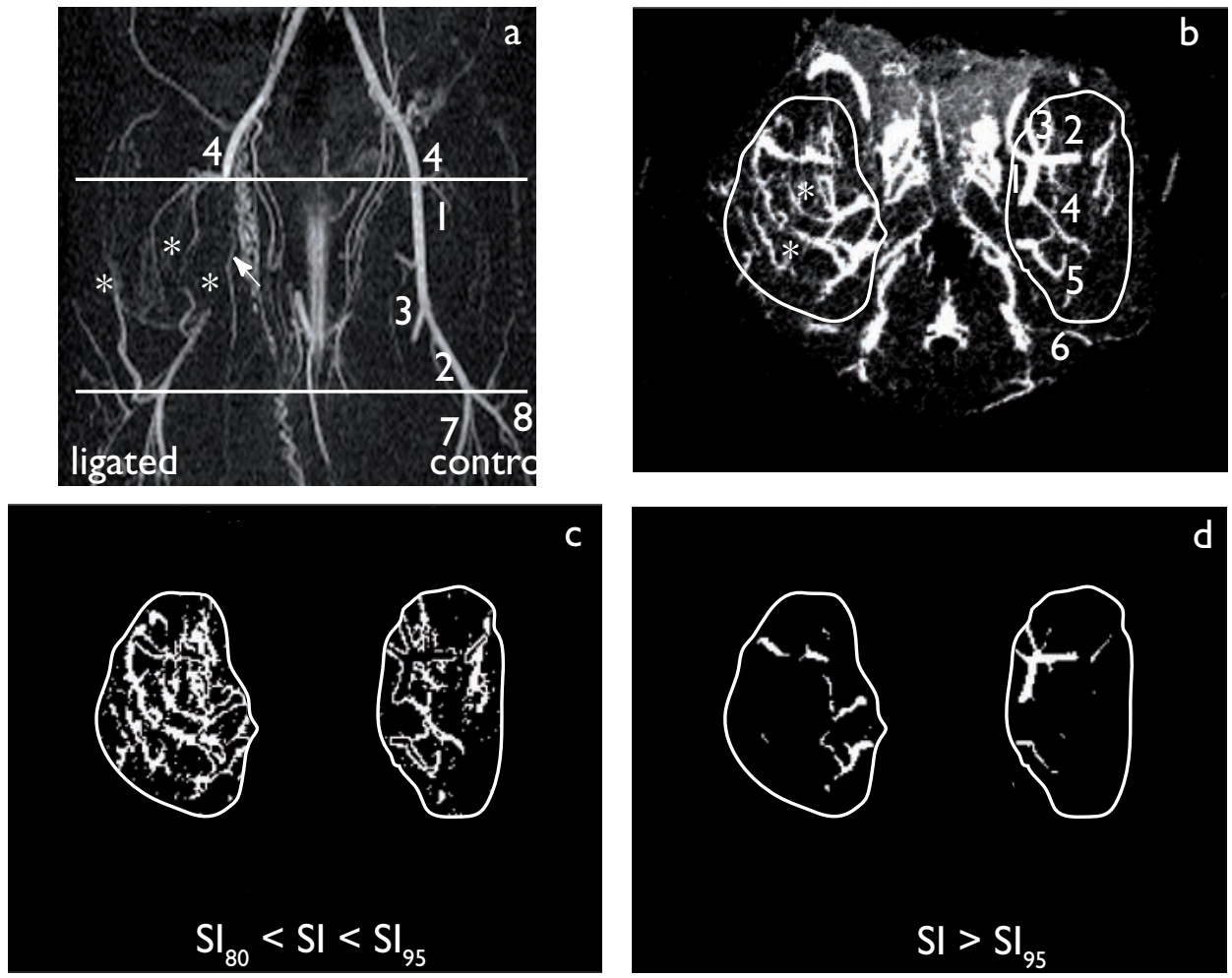

Figure 6.1: Panel a shows a coronal maximum intensity projection (MIP) of the vascular tree in a rat's hindlimb. The white lines indicate the boundaries of the stack of transverse images over which the MIP in the cranio-caudal direction in calculated (panel b). Panels $\mathrm{C}$ and $\mathrm{d}$ show the results of segmentation based on pixel signal intensities (SI) within the ROls. $\mathrm{Sl}_{80}$ and $\mathrm{Sl}_{95}$ are the signal intensities at which 80 and $95 \%$ of the pixels in the control limb have lower signal intensities. Pixels with $\mathrm{SI}$ between $\mathrm{SI}_{80}$ and $\mathrm{SI}_{95}$ are highly associated with collateral arteries in the ligated limb, whereas pixels with SI higher than $\mathrm{Sl}_{95}$ correspond to branches of the femoral arteries that feed the collaterals. White lines in panel b-d represent the regions of interest in the ligated and control limb. * indicate collateral arteries. 1: femoral artery; 2: popliteal artery; 3: saphenous artery; 4: circumflex femoral artery; 5: internal iliac artery; 6: branch of internal iliac artery, 7: cranial tibial artery; 8: caudal tibial artery. 
where $v_{e}$ is the volume fraction of the extracellular extravascular space. $C_{p}$ was approximated by a mono-exponential curve:

$$
C_{p}(t)=C_{p, \max } \cdot e^{-m t}
$$

with $C_{p, \max }$ is the maximum plasma concentration and $m$ the plasma clearance rate. Preferably, $m$ is obtained from the plasma concentration time-course in a large vessel to represent the microvascular plasma concentration in the calf muscle. However, local plasma concentrations in the calf muscles might differ due to variable delays in inflow routes. This is especially true in the ligated limb, where blood flow to the calf muscles has to traverse through small and tortuous collateral arteries instead of the direct route via an intact femoral artery. In this study, the plasma clearance rate in the tissue $m$ was therefore considered a fitting parameter. The analytical solution to equation 6.3 combined with equation 6.4 is:

$$
C_{\mathrm{t}}(t)=\frac{C_{\mathrm{p}, \max } \cdot K^{\text {trans }}}{k_{\mathrm{ep}}-m}\left(e^{m t}-e^{k_{\mathrm{ep}} t}\right)
$$

Because normalized time curves were used, $C_{p \text {,max }}, k_{e p}$, and $K^{\text {trans }}$ were unknown. Equation 6.4 is therefore reduced to:

$$
C_{\mathrm{t}}(t)=A\left(e^{m t}-e^{k t}\right)
$$

In this equation, $k_{\mathrm{ep}}$ is replaced by $k$, which represents the inflow rate, $m$ the outwash or local plasma clearance rate, and $A$ represents a scaling factor. The pharmacokinetic parameters $A, k$, and $m$ were estimated by fitting the measured concentration time curve with a non-linear Gauss-Newton sum-of-least-squares optimization algorithm in MATLAB. A maximum number of 1000 iterations and function evaluations were allowed. Lower and upper boundaries of $k$ and $m$ were set to 0 and $10 \mathrm{~min}^{-1}$, respectively, while $A$ was allowed values between 0 and 10 . The differences in $k$ and $m$ between ischemic and control muscle groups were expressed as lateralization indices (see equation 6.1) to compensate for physiological variations in hemodynamics among animals and between measurements. As a reference, the plasma clearance rate was measured in a large vascular structure in the ligated upper hindlimb, which mainly consists of the femoral vein. 


\section{Statistical analysis}

Repeated measurements analysis of variance was used to test whether the day after ligation and treatment had an effect on the arteriogenic response (expressed in $\mathrm{P}_{\text {small }}$ and $\mathrm{P}_{\text {large }}$ ), presence of edema (expressed in $\mathrm{LI}_{T 2}$ ), and DCE-MRI parameters $k$ and $m$ (expressed in $\mathrm{LI}_{m}$ and $\mathrm{LI}_{k}$ ). Interaction coefficients between variables representing day and treatment group were used to test whether the time course of each effect altered when a growth factor was administered.

Independent sample Mann-Whitney tests were performed to test the difference between the growth factor and control groups at specific time points. The changes between days 2 and 7 , and between days 7 and 21 were tested for each contrast agent separately using a paired Wilcoxon signed rank test. Effects were considered significant for $p<0.05$.

Figure 6.2 (facing page): Representative examples of maximum intensity projections in the cranio-caudal direction of time-of-flight angiograms on days 2 and 21 showing the collateral arteries formed in the upper hindlimbs, demonstrating the non-stimulated response in the control and VEGF group (upper rows), and the accelerated response to arteriogenesis therapy in the MCP-1 and FGF animals (lower rows). 
day 2
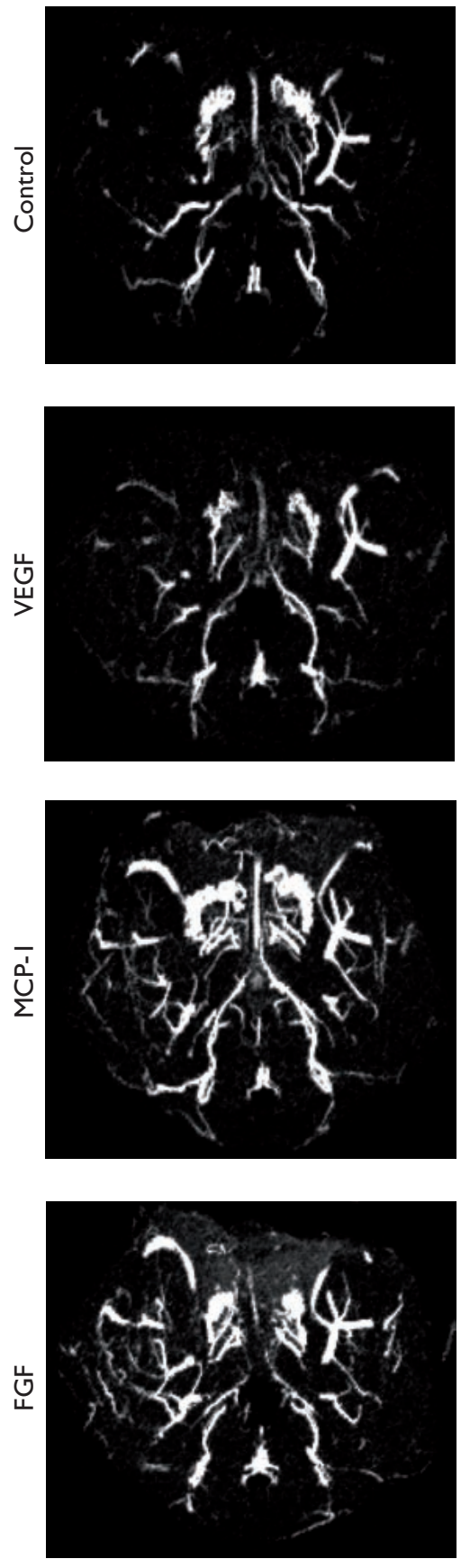

day 21
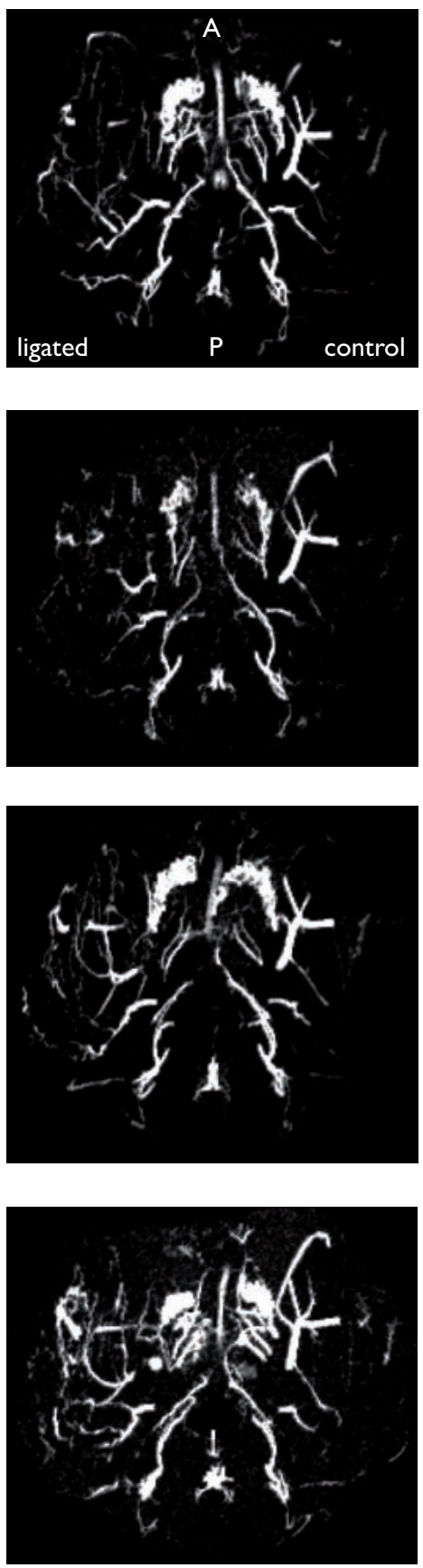


\subsection{Results}

\section{Arteriogenic response: TOF-MRA}

Figure 6.2 shows representative examples of MIPs of TOF-MR angiograms on day 2 of the upper hindlimb from the control group and the three treatment groups, showing the natural arteriogenesis response to femoral artery ligation.

The fraction of pixels representing small vessels $\left(P_{\text {small }}\right)$ was $1.3-2.5$ times higher in the ligated limb compared to the control limb (see figure 6.2). The fraction of large vessels pixels ( $P_{\text {large }}$ ) was considerably lower in the ligated limb, ranging from $0.1-0.4$ times the fraction of pixels in the control limb. Generally, both $P_{\text {small }}$ and Plarge in the ligated limb increased over time $(p<0.01)$. In the MCP-1 and FGF group, the time course of collateral artery development (expressed in $\mathrm{P}_{\text {small }}$ ) differed from the controls ( $p<0.01$ and $p=0.01$ for MCP-1 and FGF, respectively). On day $2, P_{\text {small }}$ was significantly higher for both treatment groups compared to the control group $(p=0.02)$, yet no significant change over time was observed within the treatment group was present ( $p=0.6$ (MCP-1) and $p=0.08$ (FGF) for the difference between days 2 and 21). On day 21, $P_{\text {small }}$ was lower in the MCP-1 group compared to the controls $(p=0.04)$. In the FGF and MCP-1 group, $\mathrm{P}_{\text {large }}$ was equal to the control group at all time points, and the time course of the development of the feeding branches did not differ ( $p=0.6$ and $p=0.4$, respectively).

Administration of VEGF did not affect the development of both the collaterals (expressed in $\mathrm{P}_{\text {small }}, p=0.09$ ) or their feeding arteries (expressed in $\mathrm{P}_{\text {large }}, p=0.17$ ) compared to the controls. However, Psmall was lower compared to the controls on day $7(p=0.04)$, and $\mathrm{P}_{\text {large }}$ was decreased on day $2(p=0.01)$.

\section{Angiogenic response: $T_{2}$-weighted images}

The signal intensity on the $T_{2}$-weighted images was generally higher in the ischemic limb compared to the control limb $(p<0.01$, see figure 6.4) and decreased over time $(p<0.01)$. Figure 6.5 shows the time course of the lateralization indices of the $T_{2^{-}}$ weighted signal intensities ( $\mathrm{LI}_{T 2}$ ) for all treatment groups. In rats receiving VEGF, the degree of edema did not decrease between days 2 and $21(p=0.9)$, resulting in a significantly higher $L_{T 2}$ compared to the control group on day 21 . Rats from the FGF and MCP-1 groups displayed less edema compared to the controls $(p=0.04)$, with a significant difference between control and treatment groups on day 21 ( $p=0.03$ and $p<0.01$ for MCP-1 and FGF, respectively), when no edema was found in these treatment groups. 

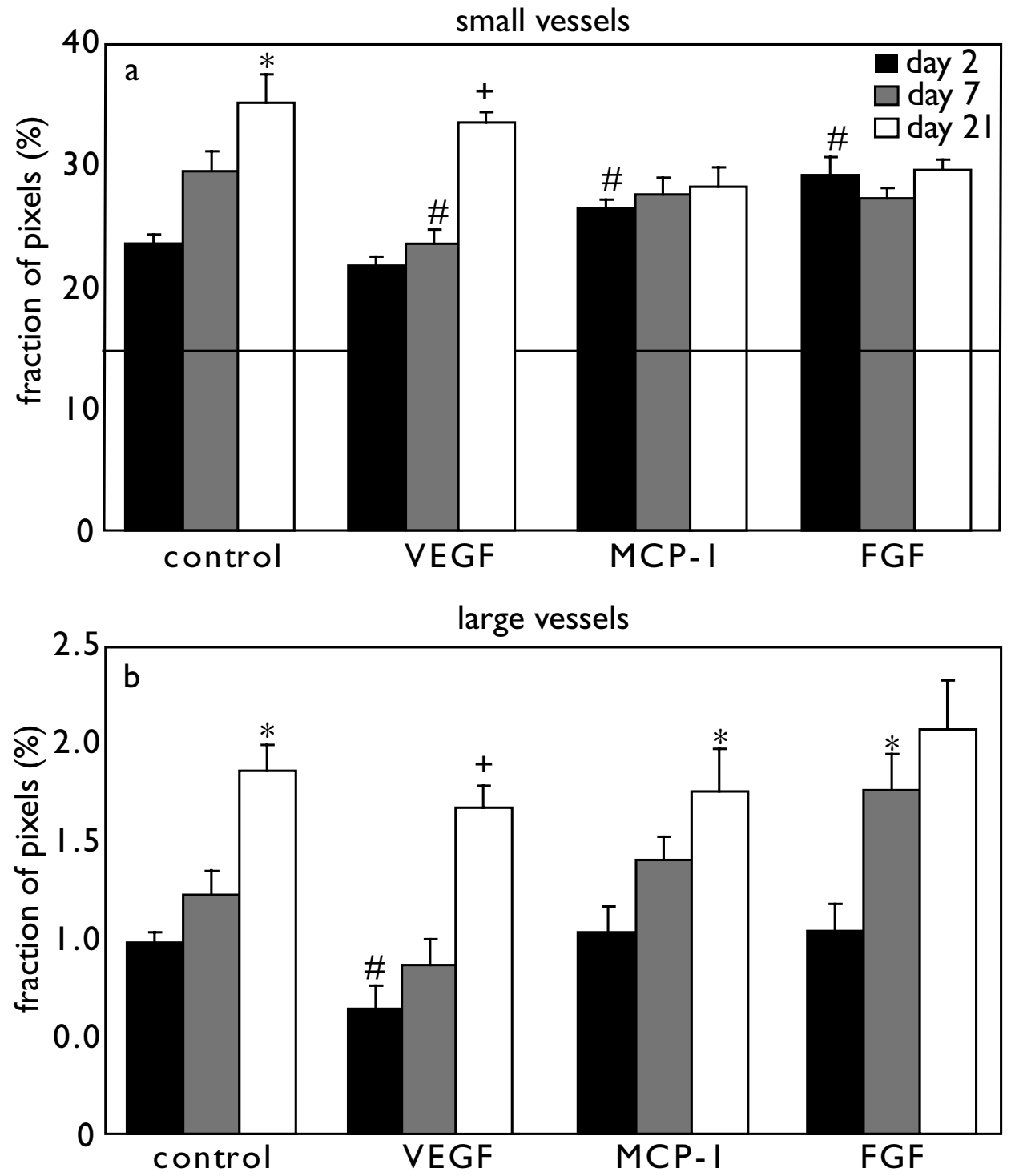

Figure 6.3: Fraction of pixels in the ligated limb representing small ( $P_{\text {small }}$, panel a) and large $\left(P_{\text {large }}\right.$, panel $\left.b\right)$ vascular structures in cranio-caudal maximum intensitiy projection of time-of-flight MR angiograms for various time points (days 2, 7 and 21) in the control and treatment groups. \# indicates a significant difference between treatment and control group $(p<0.05), *$ indicates a significant increase compared to the previous day of measurement, + indicates an increase in all animals. The black line in panel a indicates the percentage of small vessel pixels in the control limb $\left(P_{\text {small }}=15 \%\right.$ by definition $)$. The percentage of large vessel pixels in the control limb is $5 \%$. Error bars indicate standard errors of the mean. 
Chapter 6. MRI of therapeutic neovascularization

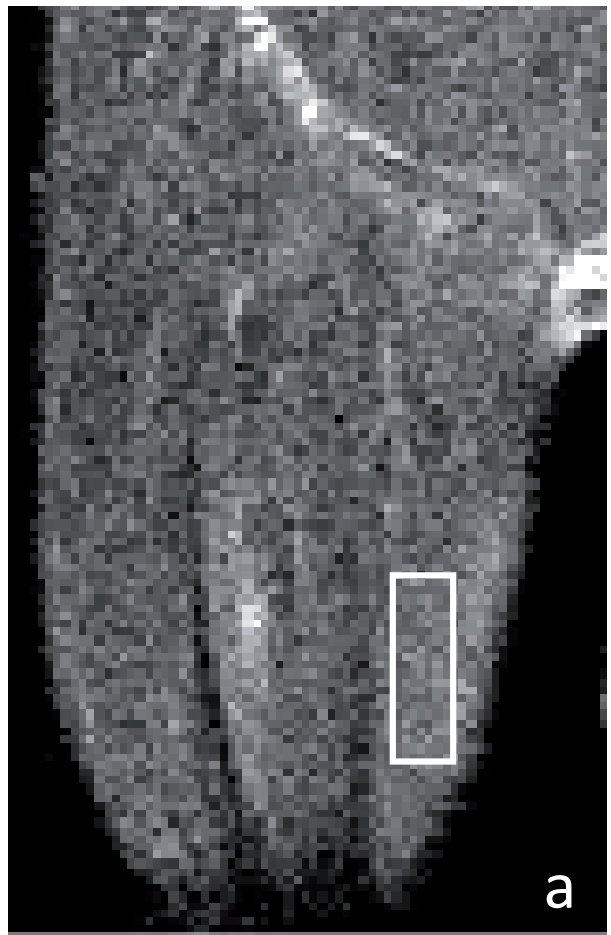

ligated

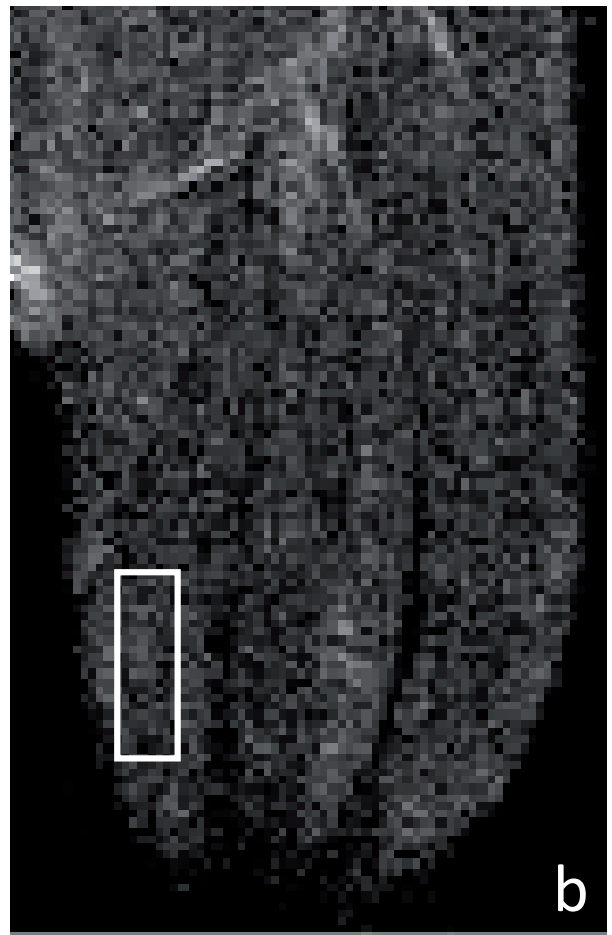

control

Figure 6.4: $T_{2}$-weighted image showing increased signal intensity in the ligated lower hindlimb (panel a) compared to the control limb (panel b). White boxes delineate the regions of interest of the ligated and control limb used for $T_{2}$-weighted signal intensity measurements and DCE-MRI. Image was obtained from a rat receiving VEGF at day 2. 


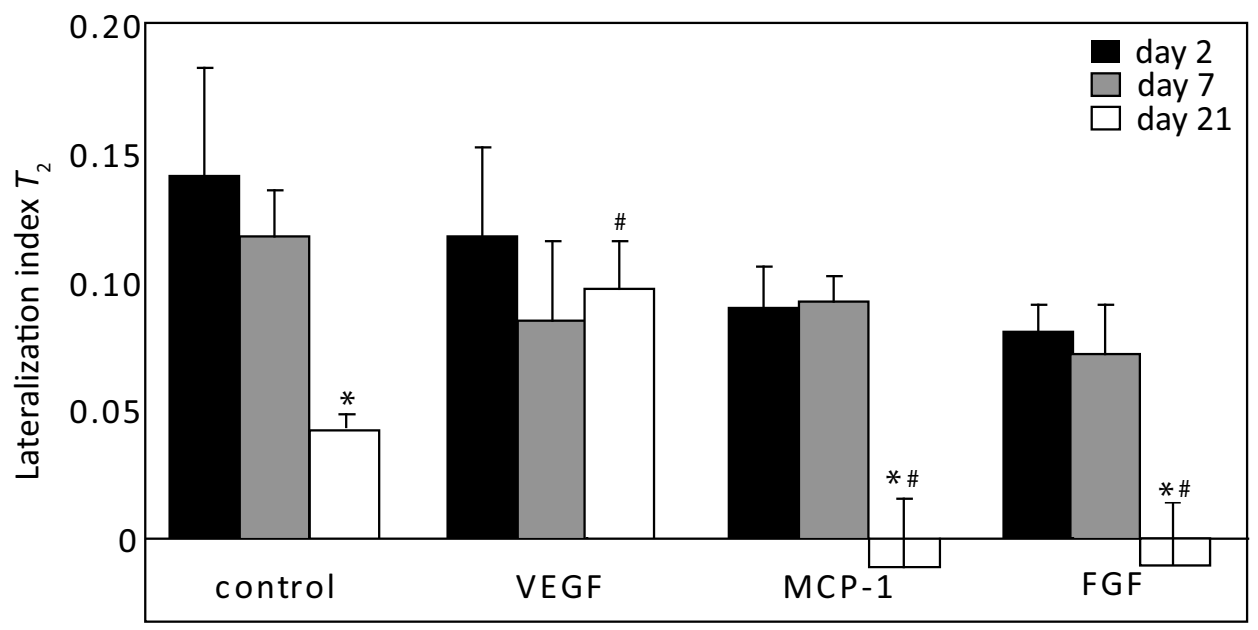

Figure 6.5: Lateralization indices of the $T_{2}$-weighted image signal intensities in the calf muscles at days 2, 7 and 21 post ligation in the control and treatment groups. \# indicate a significant difference between treatment and control group $(p<0.05)$ and $*$ indicate a significant increase compared to the previous day of measurement. Error bars indicate standard errors of the mean.

\section{Tissue perfusion: DCE-MRI}

An example of DCE-MRI signal intensity curves in the control and ischemic limb is shown in figure 6.6. The curve of the ischemic limb was characterized by a slower increase and prolonged decrease compared to the control muscle.

Local plasma clearance rate $m$ was lower in the ischemic limb compared to the control limb ( $p<0.01$, see figure 6.7) and significantly increased over time $(p<$ 0.01). Administration of MCP-1 or FGF improved the clearance (i.e. increased $m$, $p=0.01$ and $p<0.01$, respectively), with a significantly higher $L_{m}$ compared to the control group on day 2 ( $p=0.01$ and $p<0.01$ for MCP-1 and FGF, respectively) and day $7(p<0.01)$. Administration of VEGF resulted in an early improvement in $\mathrm{LI}_{m}$ compared to the controls ( $p=0.03$ on day 2 ). On day 21 post ligation, no significant differences in local plasma clearance between treatment groups were observed.

The clearance rate measured in the femoral vein was approximately three times higher compared to local plasma clearance $\left(0.16 \pm 0.04 \mathrm{~min}^{-1}\right.$ (mean \pm standard deviation) compared to $\left.0.05 \pm 0.02 \mathrm{~min}^{-1}\right)$, and did not vary significantly between days $(p=1.0)$ or treatment groups $(p>0.4)$. 
Chapter 6. MRI of therapeutic neovascularization

Inflow rate $k$ was significantly lower in the ischemic limb compared to the control $\operatorname{limb}\left(\mathrm{LI}_{k}<0\right)$ at all time points for the control $(p<0.05)$ and VEGF group $(p=$ $0.03)$. For the animals receiving MCP-1, the difference was significant on day 2 $(p=0.01)$, but not on days 7 and $21(p=0.07$ and $p=0.40$, respectively). For the FGF group, no significant difference was found on any of the days $(p>0.09)$. Standard errors on extravasation rate $k$ were large and analysis of variance did not reveal any significant effects by either time of measurement nor treatment group. 


\subsection{Discussion}

In this study, the potential of MRI for the in vivo evaluation of neovascularization therapies in hindlimb ischemia was assessed in an experimental setting. We found that MRI was able to non-invasively discriminate the effects of various treatment regimens. Furthermore, MR parameters for angiogenesis and arteriogenesis, as well as functional parameters, demonstrated time- and treatment-dependent differences between ischemic and control limbs.

\section{Arteriogenic response}

The gold standard for visualization of small collateral arteries is x-ray angiography, which has a high spatial resolution, especially when applied post mortem in combination with perfusion fixation. However, this technique is not suited for follow-up due to its invasive nature. The value of time-of-flight (TOF) MR angiography as a non-invasive alternative has already been extensively demonstrated in small animal models $[120,176]$. Because of its lower resolution, TOF-MRA may visualize only the larger collateral arteries. As flow increases rapidly with increasing diameter (flow $\sim$ diameter ${ }^{4}$, assuming laminar flow), these vessels contribute substantially more to flow recovery than the ultra-small vessels that may additionally be depicted with $x$-ray angiography [97].

Collateral arteries are usually identified and quantified manually on (rotating) maximum intensity projections (MIPs) using the Longland definition [60], which requires identification of the collateral stem, mid- and re-entry zone. Visualization of complete trajectories is challenging due to inflow artifacts and flow voids in inplane arteries [41], which makes manual identification of collateral arteries observerdependent and time-consuming. In this study, pixels in the ligated limb were qualified as collateral arteries and feeding branches based on their signal intensities. Although segmentation of individual small arteries is considered nearly impossible based on overlapping signal intensity distributions with background tissue [123,130], pixels in the ligated limb with signal intensities between certain values were found to be highly associated with visible collateral structures. Quantification of the fraction of these pixels therefore provides a fast and objective alternative to manual identification and counting of collateral arteries. Moreover, large collateral arteries, which contribute more to total flow, have more impact on the angiographic score than smaller vessels.

Analysis of TOF-MRA revealed that rats receiving growth factors that promote arteriogenesis (MCP-1 and FGF /PDGF) showed an accelerated response compared to the control animals, whereas in rats receiving VEGF, the development of collateral arteries and feeding branches showed no treatment-dependent effect. The early effects and lack of long-term advantages for MCP-1, FGF and PDGF in collateralization have previously been reported based on post-mortem $\mathrm{x}$-ray angiography $[27,151]$. 


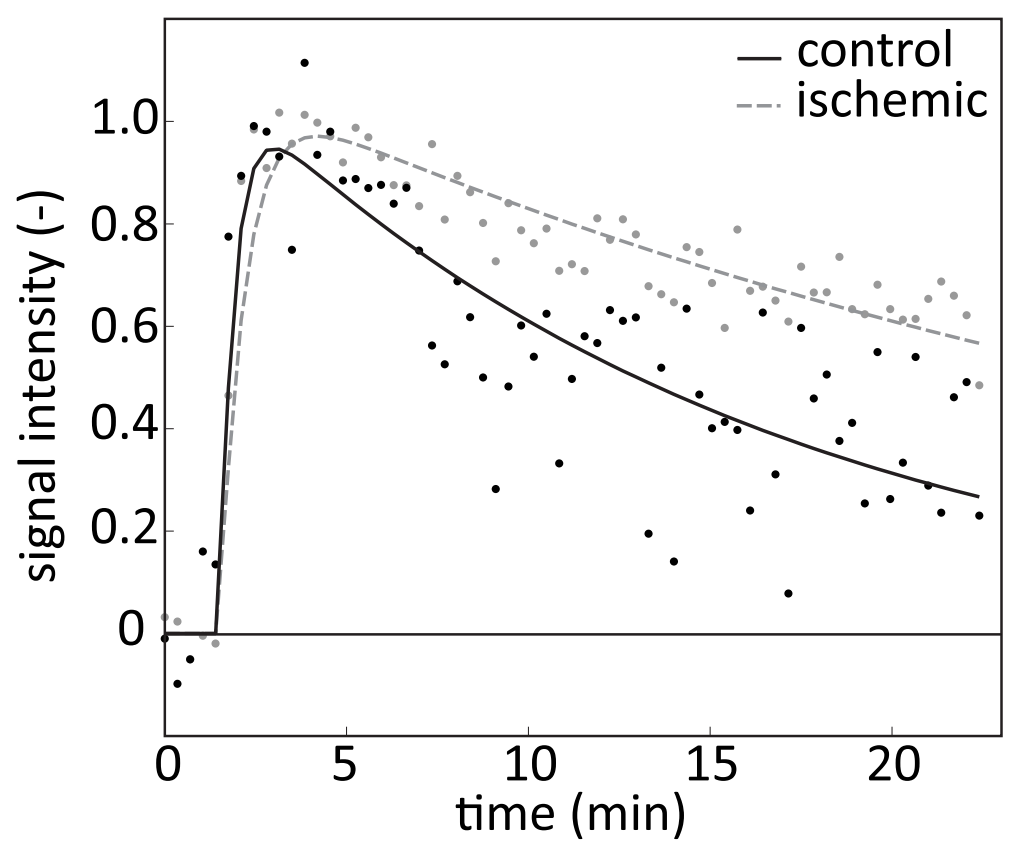

Figure 6.6: DCE-MRI signal intensity time curves in the control (black) and ischemic (grey) calf muscle. Solid and dashed lines represent the fitted curves in the control and ischemic limb, respectively. Signal intensities were normalized with respect to the minimum (set at zero) and maximum (set at 1 ) signal intensity present in the original data. Displayed data were obtained for a control rat at day 2 . 
However, no consensus is reached yet regarding the arteriogenic properties of VEGF. Some studies report no effect on the number of collateral arteries [20,32], while others found a positive effect [169]. Our data suggest that VEGF had no additional effect on top of the (natural) baseline arteriogenic response.

\section{Angiogenic response}

$T_{2}$-weighted signal intensities provide a surrogate measure for angiogenesis by visualizing the degree of edema associated with the formation of new capillaries. Immediately after ligation, hypoxia upregulates endogenous VEGF expression, which in its turn increases vascular permeability [13]. Edema as a by-product of this hyperpermeability was observed in all treatment groups during the first week after surgery. Three weeks post ligation the edema had disappeared in the MCP-1 and FGF groups, and was significantly decreased in the control animals. However, in the rats receiving exogenous VEGF, edema levels remained constant during the course of the experiment, suggesting that the therapeutically-induced angiogenic response sustained for a longer period.

Measurement of vascular permeability, rather than the resulting degree of edema, would provide a more direct measure for the angiogenic response. Ziv et al. [75] used DCE-MRI to measure permeability with a large contrast agent, and demonstrated an early increase in permeability in mice. However, both permeability and edema are surrogate measures for angiogenesis, since neither of them quantifies capillary density. As shown by Masaki et al. [177], FGF-2 therapy induces an increase in microvascular density, but as these vessels are more matured, less edema will be formed. As a result, the angiogenic response in the FGF-2/PDGF group was found to be equal or even lower compared to the controls in this study. Monitoring the angiogenic response with $T_{2}$-weighted imaging may therefore be biased towards detecting immature vessels.

Vascular hyperpermeability was found to be a crucial step in angiogenesis, as increased extravasation of plasma proteins facilitates the infiltration of endothelial cells and fibroblasts [14]. However, it might also have undesired effects when it is therapeutically enhanced. The excessive edema which is a potential result of VEGF therapy [32] is associated with tissue damage and should be avoided $[172,177]$. Apart from acting as a surrogate measure for the angiogenic response, the degree of edema should therefore also be monitored to prevent permanent tissue damage. This makes $T_{2}$-weighted imaging an indispensable tool for in vivo evaluation of neovascularization therapies.

\section{Tissue perfusion}

$T_{2}$-weighted imaging and MR angiography provide morphological information on the angiogenic and arteriogenic response, respectively. However, changes in morphology 


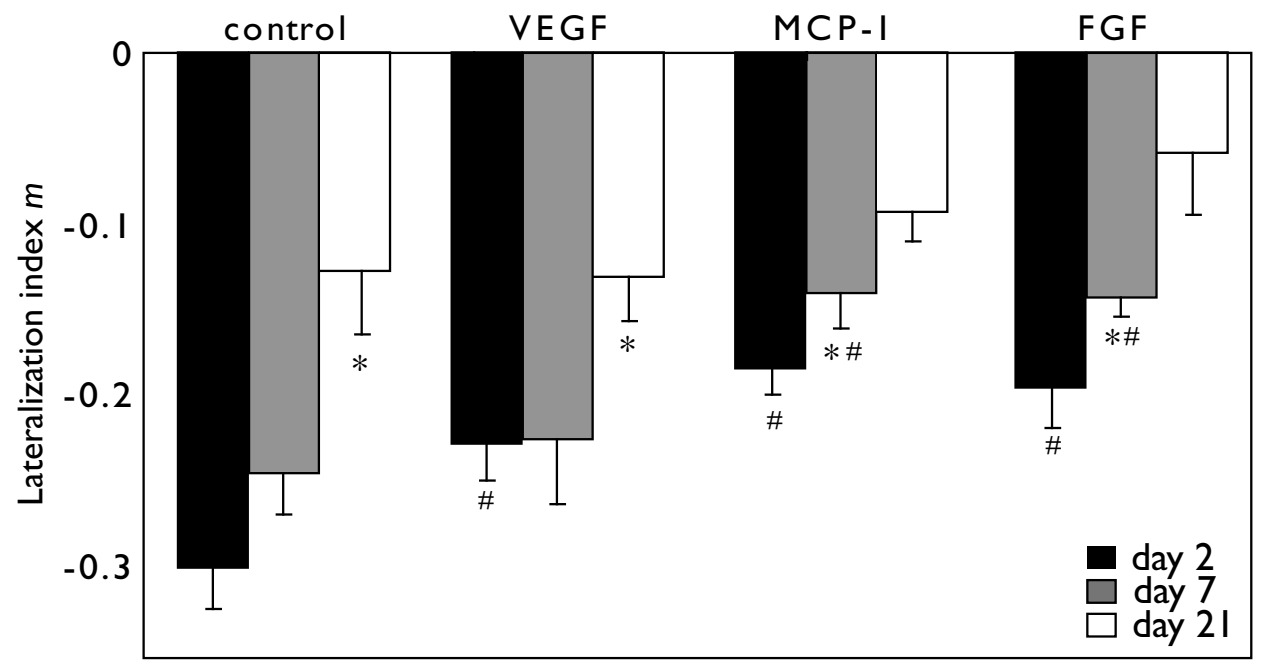

Figure 6.7: Lateralization indices of tissue clearance rate $m, 2,7$ and 21 days post ligation in the control and treatment groups. \# indicate a significant difference between treatment and control group $(p<0.05)$ and $*$ indicate a significant increase compared to the previous day of measurement. Error bars indicate standard errors of the mean.

do not necessarily result in changes in tissue perfusion, as was demonstrated previously by Fuchs et al. [61], which stresses the need for functional measurements. In this study, DCE-MRI was used to assess muscle perfusion. Functional assessment of neovascularization therapies demonstrated a positive early effect of both arteriogenic and angiogenic growth factors. Both MCP-1 and FGF/PDGF significantly improved the tissue clearance rate with respect to the untreated group during the first week post ligation, whereas the effect of VEGF was only noted on day 2.

Parameters $k$ and $m$ were estimated based on tissue enhancement curves, representing the rate of contrast agent in- and outwash, respectively. In general, $m$ reflects the plasma clearance rate, which is a characteristic of the vascular plasma concentration time course $C_{p}$ used in pharmacokinetic modeling (see equation 6.3). In this study, the clearance rate found in a large vessel was found to be 2 - 3 times faster compared to the clearance rate in the muscle microvasculature. The systemic plasma contrast agent concentration therefore appeared to be an incorrect representation of local, microvascular plasma concentration. Moreover, $m$ is likely to depend on the morphology of the supplying arterial network: compared to the control limb, it was lower in the calf muscle of the ligated limb, where blood has to flow through relatively small collateral arteries instead of a large femoral artery prior to arriving in the capillaries. The presence of collaterals will thus prolong the time to bolus arrival [97], introducing a delay in both in- and outwash as noted in both $k$ and $m$, respectively.

Inflow rate $k$, as used in this study, was derived from extravasation rate $K^{\text {trans }}$, 
which is the pharmacokinetic parameter most strongly associated with perfusion. Previous studies have shown that increased contrast agent size results in improved reliability of $K^{\text {trans }}$ estimation, even at low sampling rates [112] due to lower extravasation rates. In this study we used a medium-sized contrast agent (Gadomer), as it proved to give good results for muscle perfusion DCE-MRI in a previous study in rabbits [114]. In these animals, maximum tissue concentration was not reached within 10 minutes after contrast injection. In the present study, however, extravasation appeared to be much faster, reaching a maximum within 1 - 2 minutes. This might be attributed to a faster circulation time and increased clearance in rats compared to rabbits. As a consequence, reliable estimation of $k$ proved to be a challenge, because few data points were acquired during the upslope of the tissue concentration time curve. This resulted in a large variance of $k$, so that no effects of time and treatment could be discerned. However, early after ligation, inflow rate $k$ was systematically lower in the ischemic calf muscle compared to the control.

\section{Clinical perspective}

The efficacy of neovascularization therapies in clinical studies have often been evaluated based on long term outcomes such as limb salvage, and changes in peak walking time, ankle-brachial index or differences in ulcer healing after several weeks of therapy $[35,36]$. These are all clinically very relevant outcome measures, but they provide indirect information on the changes in vasculature in response to therapy. The MR techniques described in this study directly visualize the mechanistic physiological effect of the growth factors on the formation of new vessels. Early assessment of this vascular response enables identification of therapeutic efficacy as well as the response in individual patients. This, in turn, potentially enables individual adjustment of therapy protocol and may result in improved overall therapeutic efficacy.

\section{Conclusion}

MRI can demonstrate time- and treatment-dependent differences in terms of neovascular responses between various pharmacological neovascularization strategies in ischemic and control muscle. Time-of-flight angiography demonstrated an accelerated response of collateral artery development in rats receiving MCP-1 and FGF/PDGF, which are known to stimulate arteriogenesis. $T_{2}$-weighted imaging revealed increased levels of edema in the calf muscles of animals with a stimulated angiogenic response by VEGF. With DCE-MRI, a treatment-dependent improvement in perfusion could be measured. By combining these MR techniques, non-invasive characterization and monitoring of the vascular effects of neovascularization therapies becomes feasible. 



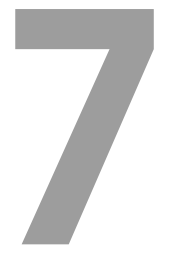

General discussion 


\subsection{Introduction}

Neovascularization, or the development of new blood vessels, plays an important role in many physiological and pathological processes. In adults, two main forms of neovascularization can be distinguished: (i) the sprouting of capillaries (angiogenesis) and (ii) the development of pre-existing arterioles into functional collateral arteries (arteriogenesis). The more extensive capillary bed formed by angiogenesis in itself may not improve tissue perfusion, as the newly formed microvessels are too small to compensate for the conductance capacity of larger vessels $[9,16]$, yet it may indirectly stimulate arteriogenesis $[17,18]$. The processes are triggered by fundamentally different conditions: angiogenesis occurs in hypoxic tissues, whereas arteriogenesis is initiated by elevations in intravascular shear stress [9]. Extensive research has identified several cytokines that play a role in neovascularization [9-11]. Some factors primarily promote either angiogenesis (e.g. VEGF $[12,26]$ ), or arteriogenesis (e.g. MCP-1 [24]), while others are involved in both processes (e.g. fibroblast growth factor (FGF-) 2 and PDGF-BB, which exhibit synergism when administered together [27]). Knowledge on the molecular mechanisms paves the way for therapeutic stimulation of neovascularization in patients with peripheral and coronary artery disease (PAD and CAD, respectively) in whom conventional, invasive revascularization therapies are not an option or unsuccessful. [27].

Animal studies have demonstrated the potential of growth factor therapy in animal hind limb and myocardial ischemia models [24, 27-34]. In clinical trials, FGF-2 has shown promising long-term results regarding therapy-dependent improvements in functional end points [35-38]. The ability to measure the primary vascular response in an early phase of therapy may therefore enable individual adjustment of treatment strategies, thereby improving therapeutic efficacy on an individual basis. The development and clinical introduction of therapeutic neovascularization would therefore benefit greatly from the development and introduction of imaging techniques that can detect and monitor early treatment effects by non-invasive evaluation of the vascular responses [39]. Due to its non-invasive nature and absence of ionizing radiation, magnetic resonance imaging (MRI) may be considered the most safe and patientfriendly option for follow-up. In addition, MRI has exquisite soft tissue contrast and is the most versatile imaging modality, which allows functional and morphological imaging within a single session. In this thesis, several MR techniques that can be used for the monitoring of neovascularization therapies were discussed. 


\subsection{Morphological imaging}

Therapies that promote neovascularization are designed to induce changes in macrovascular morphology. Angiography aims to visualize the macrovasculature, thus providing information on the number, size and location of these collateral arteries. As such, angiography can be used to assess the fundamental effects of therapy on the process of arteriogenesis.

\section{MRA, a non-invasive alternative to XRA?}

Because of its high spatial resolution and selective arterial enhancement, intra-arterial catheter $x$-ray angiography (XRA) is considered the standard of reference. However, $\mathrm{XRA}$ is an invasive procedure that requires arterial catheterization and the exposure to ionizing radiation, and is therefore not suited for monitoring of therapy-induced changes. With respect to patient safety and comfort, MR angiography (MRA) is highly favored. However, the small calibers of collateral arteries require a high spatial resolution. Hence, the main aspect that needs to be addressed when considering MRA as a viable alternative is the problem of spatial resolution, which is low compared to XRA.

When high spatial resolution is important, contrast-enhanced (CE) MRA techniques are generally preferred over non contrast-enhanced techniques such as timeof-flight (TOF) MRA, as they are considerably faster and can visualize vessels with relatively low blood flow [41]. CE-MRA images are preferably acquired during the first passage of the contrast agent bolus, when the arterial concentration is highest and veins are not yet enhanced. However, acquisition within the duration of the first-pass period limits the spatial resolution and coverage, and increases the risk of incomplete filling of vessels due to inaccurate acquisition timing. Imaging in the steady state allows higher spatial resolutions as well as complete filling of vessels with low flow [57], at the cost of decreased arterial concentration, increased surrounding tissue due to extravasation, and venous enhancement. The detrimental effect of the first two can be limited by the use of blood pool agents (BPAs), which mainly remain inside the blood vessels due to their size.

The value of steady-state MR angiography with the medium-sized BPA Gadomer for the visualization of collateral arteries was discussed in Chapter 2. Gadomer has potential in the visualization of small collateral arteries. No differences in the number of arteries between steady-state MRA and XRA were found. This is an improvement with respect to a comparable study in the same animal model [97], where a significant difference between the number of collateral arteries identified with XRA and first-pass MRA was found. Steady-state MRA therefore seems a good alternative to XRA.

An important advantage of MRA over XRA concerns the injection of the contrast agent. For XRA, the contrast agent is injected directly into the feeding artery. 
As a consequence, only collateral arteries that branch off distally from the site of injection can be visualized. In comparison, MR contrast agents are generally injected intravenously, resulting in systemic rather than local enhancement of the vasculature. Consequently, MRA provides a superior overview of the vessels in a large area.

Not all arteries can be visualized with in vivo MRA and XRA, given that the number of collateral arteries found with post mortem MRA [178] and XRA [27, 151, 169], which can depict even extremely small arteries (caliber $\sim 50 \mu \mathrm{m}$ ). Although the presence and development of these vessels is interesting from a scientific point of view, the question is whether the collaterals which are missed out on with in vivo MRA contribute substantially to the restoration of perfusion. As flow increases rapidly for increasing diameter (flow $\sim$ diameter ${ }^{4}$, assuming laminar flow), smaller vessels contribute substantially less to flow recovery and are therefore clinically less relevant than the vessels that are already demonstrable with MRA [97]. Consequently, although in vivo techniques may not depict all collateral arteries, it expectedly visualizes the clinically important ones.

\section{Contrast agent size in CE-MRA}

In Chapter 2, we demonstrated that the blood pool agent Gadomer is suitable for MRA of collateral arteries. The use of Gadomer noticeably improved contrast-tonoise characteristics compared to the clinically used small contrast agent Gd-DTPA, while the dose could be reduced. Interestingly, the resulting improved visibility of collateral arteries did not result in an increase in number of identified collaterals, yet it appreciably facilitated the identification of collateral arteries. Apparently, the quality of the Gd-DTPA angiograms was sufficiently high, leaving little room for improvement for Gadomer. This was supported by the finding that no significant difference was observed with the number of collateral arteries visible on $\mathrm{x}$-ray angiograms. An important question is whether blood pool agents have additional value compared to small contrast agents in CE-MRA of collateral arteries. First, the importance of facilitated identification of collateral arteries in clinical practice should not be underestimated. When less time is required to analyze the data, the technique is more likely to find its way into clinic practice. Second, in human studies, the spatial coverage needs to be much larger than in rabbit hind limb models. It would be interesting to see if the potential of BPAs becomes more pronounced when applied in clinical trials. As the value of BPAs in diagnostic steady-state CE-MRA is wellestablished $[57,59,179]$, this might well be the case.

\section{Image analysis}

As the diameters of collateral arteries $(1 \mathrm{~mm}$ or smaller) are generally lower than the pixel size in clinical imaging, vessels are often represented by no more than a 
single pixel. The contrast between these individual vascular pixels and background may be small, which makes it difficult to follow the collateral artery from its origin to the re-entry point, or determine the number of vessels crossing a plane [180]. As a consequence, manual identification and quantification of collateral arteries is time-consuming and highly subjective, and does not differentiate between small and larger collaterals.

In Chapter 3, a more objective and faster method for the quantification of arteriogenesis with MR angiograms was proposed. Characteristics of the signal intensity distribution of TOF-MR angiograms were shown to correspond to the presence of small (approximately $0.5 \mathrm{~mm}$ ) and large arterial structures. These novel output parameters correlated well with the number of collateral arteries found by manual identification. The method seemed more sensitive to changes in vasculature than manual identification, as it could differentiate the time courses of small and ultrasmall collateral artery development.

The analysis strategy was developed and tested on TOF-MR angiograms, which are characterized by the very effective suppression of background signal and, if applied adequately, selective arterial enhancement. Generally, no difference can be observed between various types of background tissue, such as muscle or lipid-rich bone. As a consequence, any deviations from the normally distributed background signal intensity must be related to the presence of arteries. To obtain similar conditions in CE-MRA, pre-contrast images should be subtracted from the contrast-enhanced data. This step is more likely to be successful when blood pool agents are used, as they improve contrast between collateral arteries and background, as demonstrated in Chapter 2. Blood pool agents may therefore not only facilitate manual identification, but also increase the success of automated analysis strategies. 


\subsection{Functional imaging}

Ultimately, neovascularization therapies aim to increase blood flow distally to the occlusion or stenosis, and thus relieve tissue ischemia. As clinically relevant outcomes like peak walking time, exercise tolerance and wound healing are related to tissue perfusion, direct measurement of microvascular flow would provide a more objective, short-term outcome measure for tissue function. A promising non-invasive tool to assess muscle perfusion is dynamic contrast-enhanced (DCE) MRI. This technique is based on measurement of the passage and extravasation of a contrast agent, from which perfusion-related parameters can be estimated with pharmacokinetic modeling or model-free approaches. DCE-MRI is already an established diagnostic method in oncology [68-70], and has also shown promising results for monitoring anti-cancer therapy [71-74]. Chapters 4 and 5 discussed the optimization of DCE-MRI image acquisition and analysis for improved muscle perfusion measurements.

\section{Contrast agent size in DCE-MRI}

DCE-MRI is most commonly performed with small contrast agents (SCAs). However, simulation studies have shown that their high extravasation rates require fast imaging protocols [87-89], at the cost of spatial coverage and/or resolution. DCE-MRI would therefore benefit from the use of larger contrast agents, which have reduced extravasation rates [90]. Such contrast agents would allow longer dynamic acquisition times, which could be employed to improve image quality. In Chapters 4 and 5, the application of larger, medium-sized contrast agents (MCA) in muscle perfusion measurements with DCE-MRI was investigated.

\section{Image acquisition}

Since most DCE-MRI acquisition protocols are optimized for SCAs, the optimal experimental settings for MCAs have to be re-defined. Chapter 4 investigated which acquisition requirements should be met for optimal parameter estimation with MCAs, and whether the use of MCAs would result in improved reliability of parameter estimation. Monte Carlo simulations showed that MCAs such as Gadomer provide decreased bias and variance of pharmacokinetic parameters compared to SCAs such as Gd-DTPA. Due to their lower extravasation rate, MCAs allowed lower sampling frequencies compared to SCAs, while obtaining higher reliability in the estimation of extravasation rate $K^{\text {trans }}$ and plasma fraction $v_{p}$. For optimal parameter reliability, total acquisition time should be somewhat longer for the MCA ( 5 versus 2 minutes for the SCA), but this should not impose too high a burden on patient comfort or magnet time. 


\section{Image analysis}

Considering the contrast agent-specific requirements on sampling rate and acquisition time defined in Chapter 4, the application of DCE-MRI with MCAs in an experimental setting was subsequently explored in Chapter 5 , with a focus on data analysis optimization. In the past decades, several pharmacokinetic models describing the relation between plasma and tissue concentration in terms of physiologically relevant parameters have been proposed $[70,81-84]$. Selecting the most appropriate model to describe the acquired data is an important step in DCE-MRI. Analysis of the quality of fit, as proposed in similar studies $[150,156]$, may be the most straightforward way to assess model validity, yet it does not evaluate parameter reliability. One could argue that as long as the uncertainty on $K^{\text {trans }}$, which is a surrogate measure for microvascular flow $[85,86]$, is small, the model used is appropriate. However, the use of a model including parameters which cannot be estimated reliably may have a detrimental effect on the estimation of $K^{\text {trans }}$ due to parameter interdependency. We therefore feel that estimation uncertainties and parameter interdependencies should also be used when assessing the validity of a model. In pharmacokinetic modeling, the principle of Ockham's razor should be applied: the simplest model which adequately describes the data is most likely to be the best. This notion is supported by our findings regarding the detection of perfusion differences, which is most sensitive when the analysis strategy is tailored for each contrast agent and each situation individually.

Another way to derive perfusion-related parameters from the tissue concentration time curve is to describe the characteristics of the curve itself. Examples of these so-called model-free parameters are area-under-curve (AUC), time-to-peak and initial slope. As described in Chapter 5, both the physiological meaning and sensitivity to perfusion differences depends on the definition of AUC. Comparably to model-based analysis, the pharmacokinetic behavior of the contrast agent in the tissue of interest should therefore be considered when defining the parameters. When the optimal, or most sensitive, definition is used, model-free parameter analysis proved to be equally sensitive to perfusion differences as model-based parameter $K^{\text {trans }}$. From a practical point of view, model-free methods are easier to implement. However, the opportunity of standardization and quality control greatly favor model-based analysis.

\section{Detection of perfusion differences}

In Chapter 5, pathophysiological (between ischemic and control limbs) and physiological (between red and white muscle) microvascular differences could be detected with both the medium-sized and small contrast agent. No differences in sensitivity between the MCA and SCA were observed, yet the spatial resolution was appreciably improved for the MCA, without loss of signal-to-noise or contrast ratios. The decreased extravasation rates of MCAs may be especially beneficial in cardiac DCE- 
MRI, where the use of Gadomer substantially prolongs the time frame during which ischemic and healthy myocardium can be differentiated [165]. The consequential increase in spatial resolution is clinically relevant, because detailed information of the spatial extent (transmurality) of the infarcted area is an important predictor of recovery [166].

Contrast-enhanced perfusion techniques are generally limited to measure rest perfusion. As rest perfusion is only affected in more progressed phases of PAD and CAD, stress-induced DCE-MRI measurements could provide additional information. An example for such a protocol was proposed by Thompson et al. [78]. However, the reactions to stress are fast, which requires rapid image acquisition. With small contrast agents, extravasation rapidly reaches a maximum, which limits the time during which alterations in stress can be applied. It would be interesting to investigate whether the longer period during which extravasation of MCAs occurs could be used to extend the protocol, for example with multiple cuff compressions. In addition, with the use of large contrast agents reliable estimation of $v_{p}$ becomes feasible. During reactive hyperemia, healthy tissue displays increased microvascular dilation, whereas in ischemic tissue, this mechanism is impaired. The degree of variation in $v_{p}$ may therefore be a good representation of the flow reserve.

\section{Comparison to other functional imaging techniques}

Apart from DCE-MRI, several other MR techniques are available for perfusion measurements. Examples of non-contrast-enhanced methods include blood oxygenation level-dependent (BOLD) MRI, which can be used to determine the perfusion capacity of skeletal muscle and myocardium $[63-65,181]$. In BOLD MRI, changes in the ratio of oxygenated and deoxygenated hemoglobin are observed as signal changes on a $T_{2}^{*}$-weighted image. In reaction to stress, local perfusion increases rapidly in healthy tissue. As the increase in blood supply exceeds the oxygen demand, the oxyhemoglobin level rises accordingly. Quantification of the dynamic changes in signal intensity thus provides a measure for the flow reserve, which is impaired in patients with PAD and CAD $[64,181,182]$. The concept of BOLD imaging is fundamentally different from DCE-MRI, as it measures the flow reserve rather than flow itself. However, moderate to good correlations between BOLD parameters and flow measurements $[183,184]$ have been demonstrated.

A second interesting MR-based perfusion technique is arterial spin labeling (ASL). To obtain perfusion-based contrast, spins are labeled proximal to the imaging plane. Because of flow, labeled spins in blood progress downstream. The amount of labeled spins that after a short period arrive in the microvessels in the imaging plane is directly proportional to perfusion [185]. This enables truly quantitative perfusion measurements in both skeletal muscle [186] and myocardium $[187,188]$, providing a good correlation with other perfusion techniques [188-190].

Compared to DCE-MRI, both BOLD and ASL have several disadvantages. First, 
they are characterized by fairly poor signal-to-noise intensity and contrast-to-noise characteristics. The sensitivity of these techniques to perfusion differences is low, so stress-inducing protocols are indispensable. Second, because very fast imaging is required, both the spatial resolution and coverage are more limited. Finally, the analysis of BOLD and ASL data is complex and as the techniques are mainly developed for brain perfusion, few processing tools are available yet. 


\subsection{Comprehensive MRI}

In Chapter 6, we showed how multiple MR techniques can be combined to provide morphological and functional information on the angiogenic and arteriogenic response. Time-of-flight MR angiograms, analyzed with the automated method proposed in Chapter 3, revealed an increased arteriogenic response in rats receiving MCP-1 and FGF/PDGF, which are known to stimulate the formation of collateral arteries. $T_{2}$-weighted images provided information of the levels of edema in the calf muscle, and can thus serve as a measure for the angiogenic response. The measurements revealed a prolonged increase in edema in rats receiving the angiogenesis stimulator VEGF. With DCE-MRI, tissue perfusion was assessed. An accelerated improvement in tissue clearance in on the ligated side was observed in all treatment groups.

To get a complete view on the vascular response to neovascularization therapies, merely assessing either morphological or functional changes is not sufficient. When therapeutic efficacy in terms of functional improvements is hampered, it is necessary to explore if this can be attributed to an insufficient arteriogenic response to neovascularization therapy. It may be that although the direct vascular response to therapy is satisfactory, the changes in morphology do not necessarily result in changes in tissue perfusion, as was demonstrated previously by Fuchs et al. [61]. In that case, further examination of the (micro-) vascular function is needed.

As shown in this thesis, MRI is a very versatile imaging modality. It enables characterization of various modes of neovascularization in a hind limb ischemia model, and allows comprehensive non-invasive monitoring of the efficacy of neovascularization therapies in terms of vascular responses. An overview of the MR techniques dedicated to functional and morphological assessment of neovascularization on a macro- and microvascular level, as discussed in this thesis, is provided in figure 7.1. MR angiography can be used to visualize morphological changes in macrovasculature. $T_{2}$-weighted imaging is a fast, yet effective method to assess the presence of edema and tissue damage. Traditionally, DCE-MRI can be used to measure tissue perfusion. However, with the introduction of large contrast agents, it may also become a more sensitive alternative to ASL and BOLD for measuring the perfusion reserve. 
7.4 Comprehensive MRI

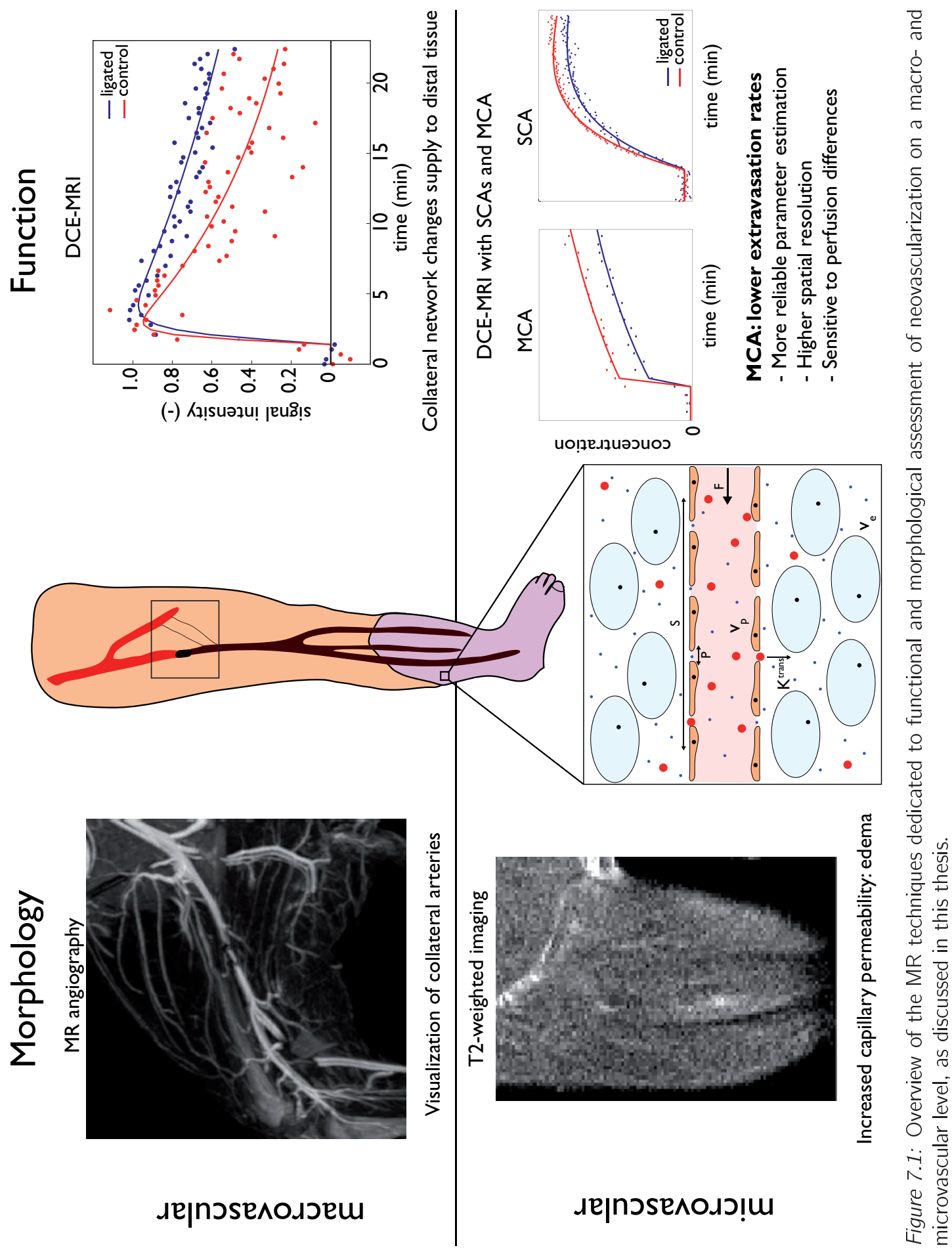




\subsection{From animal model to clinical application}

The next step towards clinical application is the translation from animal model to patient. The MR techniques described in this thesis can all be implemented on clinical MR systems. The hind limb ischemia model [151] used in this thesis is a valid model for neovascularization in response to arterial occlusion in both the myocardium and skeletal muscle. A general advantage of the use of animal models is the standardized procedures and controlled environment. By applying the ligation unilaterally, the contra-lateral limb is available as an intra-individual healthy control. In patients, however, the disease usually affects both legs, which makes quantitative measurements far more important than in the animal model.

The hind limb ischemia model serves as a model for neovascularization but is not an adequate model for the pathology of PAD. No atherosclerosis is present in the animals and the occlusion of the femoral artery is acute, rather than gradual. As a result, the response to intervention is strong, and easily detectable. Consequently, in Chapter 6 , the control animals displayed a high degree of arteriogenesis which could hardly be improved by administration of growth factors. The natural response is expected to be considerably smaller in patients, especially those who also suffer from diabetes [191]. Although the MR techniques described in this thesis can all be implemented on clinical MR systems, the value in the assessment of neovascularization requires further evaluation.

This thesis shows the potential of larger contrast agents in cardiovascular MRI. However, the agent used in our studies, Gadomer, is not available for clinical use. The value of gadofosveset trisodium [163], which currently is the only clinically available blood pool agent in CE-MRA is undisputed $[59,192]$. However, its value in DCE-MRI is limited. Gadofosveset itself has a molecular weight of $1.0 \mathrm{kDa}$, but after non-covalent binding to albumin, the molecular weight becomes $70 \mathrm{kDa}$. In human plasma, $80-96 \%$ of the contrast agent is bound [163], yet during the first passage of the contrast agent, a large fraction (74\% [104]) is still unbound and extravasates more rapidly. The variation in fraction of bound contrast agent makes pharmacokinetic modeling complicated, resulting in a poor correlation with histology [74]. Nevertheless, gadofosveset should not be written off as a contrast agent for DCE-MRI.

A good correlation between signal enhancement rate and myocardial blood flow during a first-pass DCE-MRI experiment has already been demonstrated [193, 194]. However, it can be questioned whether gadofosveset can actually be considered a large contrast agent during these experiments. Further investigation of the pharmacokinetic properties and adapted data analysis strategies are needed to enable quantitative DCE-MRI with gadofosveset. 


\subsection{The future of therapeutic neovascularization}

The ultimate goal of the development of MR techniques dedicated to assessing neovascularization is to support the improvement and clinical introduction of therapeutic neovascularization. Clinical neovascularization is still in its infancy, with many problems to be addressed. Important unanswered questions include the way of administration of the compounds [118], selection of the optimal agent, prevention of adverse side effects such as plaque destabilization and evaluation of therapeutic efficacy.

MRI has the potential to play an important part in both preclinical and clinical studies. In animal studies, MR techniques can be used for non-invasive monitoring of the effect of new compounds. As it allows longitudinal monitoring, fewer animals are needed. In addition to the conventional techniques described in this thesis, molecular imaging may play an important role in this stage of research. Molecular imaging provides in vivo visualization of biological processes and molecular pathways [195] by binding contrast agents to disease-specific markers. This provides early visualization of biochemical changes. For angiogenesis, many markers and corresponding ligands have been developed $[15,196]$ and successful application in cardiovascular disease models has already been described $[197,198]$. In contrast, molecular MRI of arteriogenesis is virtually non-existent. Whether molecular MRI could have added value in monitoring arteriogenesis may be questioned, as the effects are visible on a macroscopic scale. However, it would be interesting to see if there is potential for molecular imaging in explaining mechanisms why the arteriogenic response is hampered in certain pathologies diabetes.

This thesis aims to contribute to the clinical introduction of neovascularization therapies by providing techniques that enable early evaluation of therapy, and consequently improving in outcome of patients with PAD and CAD. Attanasio and Snell [117] expressed that 'the fate of therapeutic neovascularization as a viable treatment option for patients with PAD and CAD rests upon the continued enthusiasm and perseverance of bench and clinical researchers'. In our opinion, this includes radiologists and medical physicists, and the MR community should therefore continue to contribute to this goal by constantly improving morphological and functional imaging techniques. 



\section{References}

[1] Norgren L., Hiatt W. R., Dormandy J. A., et al. Inter-Society Consensus for the Management of Peripheral Arterial Disease (TASC II) Eur J Vasc Endovasc Surg. 2007;33 Suppl 1:S1-75.

[2] Eskelinen E., Lepantalo M., Hietala E. M., et al. Lower limb amputations in Southern Finland in 2000 and trends up to 2001 Eur J Vasc Endovasc Surg. 2004;27:193-200.

[3] Seiler C.. The human coronary collateral circulation Eur J Clin Invest. 2010;40:465-76.

[4] Zbinden S., Zbinden R., Meier P., Windecker S., Seiler C.. Safety and efficacy of subcutaneous-only granulocyte-macrophage colony-stimulating factor for collateral growth promotion in patients with coronary artery disease $J$ Am Coll Cardiol. 2005; 46:1636-42.

[5] Elsman P., Hof A. W., Boer M. J., et al. Role of collateral circulation in the acute phase of ST-segment-elevation myocardial infarction treated with primary coronary intervention Eur Heart J. 2004;25:854-8.

[6] Sabia P. J., Powers E. R., Ragosta M., Sarembock I. J., Burwell L. R., Kaul S.. An association between collateral blood flow and myocardial viability in patients with recent myocardial infarction N Engl J Med. 1992;327:1825-31.

[7] Kodama K., Kusuoka H., Sakai A., et al. Collateral channels that develop after an acute myocardial infarction prevent subsequent left ventricular dilation J Am Coll Cardiol. 1996;27:1133-9.

[8] Meier P., Gloekler S., Zbinden R., et al. Beneficial effect of recruitable collaterals: a 10-year follow-up study in patients with stable coronary artery disease undergoing quantitative collateral measurements Circulation. 2007;116:975-83.

[9] Heil M., Eitenmuller I., Schmitz-Rixen T., Schaper W.. Arteriogenesis versus angiogenesis: similarities and differences J Cell Mol Med. 2006;10:45-55.

[10] Carmeliet P.. Mechanisms of angiogenesis and arteriogenesis Nat Med. 2000;6:38995.

[11] Conway E. M., Collen D., Carmeliet P.. Molecular mechanisms of blood vessel growth Cardiovasc Res. 2001;49:507-21.

[12] Yancopoulos G. D., Davis S., Gale N. W., Rudge J. S., Wiegand S. J., Holash J.. Vascular-specific growth factors and blood vessel formation Nature. 2000;407:242-8.

[13] Dvorak H. F., Brown L. F., Detmar M., Dvorak A. M.. Vascular permeability factor/vascular endothelial growth factor, microvascular hyperpermeability, and angiogenesis Am J Pathol. 1995;146:1029-39.

[14] Dvorak H. F., Nagy J. A., Feng D., Brown L. F., Dvorak A. M.. Vascular permeability factor/vascular endothelial growth factor and the significance of microvascular hyperpermeability in angiogenesis Curr Top Microbiol Immunol. 1999;237:97-132.

[15] Oostendorp M., Post M. J., Backes W. H.. Vessel growth and function: depiction with contrast-enhanced MR imaging Radiology. 2009;251:317-35. 
[16] Scholz D., Ziegelhoeffer T., Helisch A., et al. Contribution of arteriogenesis and angiogenesis to postocclusive hindlimb perfusion in mice J Mol Cell Cardiol. 2002;34:775-87.

[17] Helisch A., Schaper W.. Arteriogenesis: the development and growth of collateral arteries Microcirculation. 2003;10:83-97.

[18] Scholz D., Cai W. J., Schaper W.. Arteriogenesis, a new concept of vascular adaptation in occlusive disease Angiogenesis. 2001;4:247-57.

[19] Ito W. D., Arras M., Scholz D., Winkler B., Htun P., Schaper W.. Angiogenesis but not collateral growth is associated with ischemia after femoral artery occlusion Am $J$ Physiol. 1997;273:H1255-65.

[20] Deindl E., Buschmann I., Hoefer I. E., et al. Role of ischemia and of hypoxiainducible genes in arteriogenesis after femoral artery occlusion in the rabbit Circ Res. 2001;89:779-86.

[21] Pipp F., Boehm S., Cai W. J., et al. Elevated fluid shear stress enhances postocclusive collateral artery growth and gene expression in the pig hind limb Arterioscler Thromb Vasc Biol. 2004;24:1664-8.

[22] Shyy Y. J., Hsieh H. J., Usami S., Chien S.. Fluid shear stress induces a biphasic response of human monocyte chemotactic protein 1 gene expression in vascular endothelium Proc Natl Acad Sci U S A. 1994;91:4678-82.

[23] Wang D. L., Wung B. S., Shyy Y. J., et al. Mechanical strain induces monocyte chemotactic protein-1 gene expression in endothelial cells. Effects of mechanical strain on monocyte adhesion to endothelial cells Circ Res. 1995;77:294-302.

[24] Ito W. D., Arras M., Winkler B., Scholz D., Schaper J., Schaper W.. Monocyte chemotactic protein-1 increases collateral and peripheral conductance after femoral artery occlusion Circ Res. 1997;80:829-37.

[25] Heil M., Schaper W.. Influence of mechanical, cellular, and molecular factors on collateral artery growth (arteriogenesis) Circ Res. 2004;95:449-58.

[26] Neufeld G., Cohen T., Gengrinovitch S., Poltorak Z.. Vascular endothelial growth factor (VEGF) and its receptors Faseb J. 1999;13:9-22.

[27] Cao R., Brakenhielm E., Pawliuk R., et al. Angiogenic synergism, vascular stability and improvement of hind-limb ischemia by a combination of PDGF-BB and FGF-2 Nat Med. 2003;9:604-13.

[28] Sato K., Laham R. J., Pearlman J. D., et al. Efficacy of intracoronary versus intravenous FGF-2 in a pig model of chronic myocardial ischemia Ann Thorac Surg. 2000;70:2113-8.

[29] Hopkins S. P., Bulgrin J. P., Sims R. L., Bowman B., Donovan D. L., Schmidt S. P.. Controlled delivery of vascular endothelial growth factor promotes neovascularization and maintains limb function in a rabbit model of ischemia J Vasc Surg. 1998;27:88694; discussion 895.

[30] Wafai R., Tudor E. M., Angus J. A., Wright C. E.. Vascular effects of FGF-2 and VEGF-B in rabbits with bilateral hind limb ischemia J Vasc Res. 2009;46:45-54. 
[31] Srivastava S., Terjung R. L., Yang H. T.. Basic fibroblast growth factor increases collateral blood flow in spontaneously hypertensive rats Am J Physiol Heart Circ Physiol. 2003;285:H1190-7.

[32] Hershey J. C., Baskin E. P., Corcoran H. A., et al. Vascular endothelial growth factor stimulates angiogenesis without improving collateral blood flow following hindlimb ischemia in rabbits Heart Vessels. 2003;18:142-9.

[33] Schirmer S. H., Buschmann I. R., Jost M. M., et al. Differential effects of MCP-1 and leptin on collateral flow and arteriogenesis Cardiovasc Res. 2004;64:356-64.

[34] Laham R. J., Rezaee M., Post M., et al. Intrapericardial delivery of fibroblast growth factor-2 induces neovascularization in a porcine model of chronic myocardial ischemia J Pharmacol Exp Ther. 2000;292:795-802.

[35] Lederman R. J., Mendelsohn F. O., Anderson R. D., et al. Therapeutic angiogenesis with recombinant fibroblast growth factor-2 for intermittent claudication (the TRAFFIC study): a randomised trial Lancet. 2002;359:2053-8.

[36] Nikol S., Baumgartner I., Van Belle E., et al. Therapeutic angiogenesis with intramuscular NV1FGF improves amputation-free survival in patients with critical limb ischemia Mol Ther. 2008;16:972-8.

[37] Simons M., Annex B. H., Laham R. J., et al. Pharmacological treatment of coronary artery disease with recombinant fibroblast growth factor-2: double-blind, randomized, controlled clinical trial Circulation. 2002;105:788-93.

[38] Laham R. J., Chronos N. A., Pike M., et al. Intracoronary basic fibroblast growth factor (FGF-2) in patients with severe ischemic heart disease: results of a phase I open-label dose escalation study J Am Coll Cardiol. 2000;36:2132-9.

[39] Simons M.. Angiogenesis: where do we stand now? Circulation. 2005;111:1556-66.

[40] Ersoy H., Rybicki F. J.. MR angiography of the lower extremities AJR Am J Roentgenol. 2008;190:1675-84.

[41] Ho K. Y., Leiner T., Haan M. W., Engelshoven J. M.. Peripheral MR angiography Eur Radiol. 1999; 9:1765-74.

[42] Leiner T.. Magnetic resonance angiography of abdominal and lower extremity vasculature Top Magn Reson Imaging. 2005;16:21-66.

[43] Meaney J. F.. Magnetic resonance angiography of the peripheral arteries: current status Eur Radiol. 2003;13:836-52.

[44] Rofsky N. M., Adelman M. A.. MR angiography in the evaluation of atherosclerotic peripheral vascular disease Radiology. 2000;214:325-38.

[45] Botnar R. M., Stuber M., Danias P. G., Kissinger K. V., Bornert P., Manning W. J.. Coronary magnetic resonance angiography Cardiol Rev. 2001;9:77-87.

[46] Stuber M., Weiss R. G.. Coronary magnetic resonance angiography J Magn Reson Imaging. 2007;26:219-34. 
[47] Li D., Zheng J., Weinmann H. J.. Contrast-enhanced MR imaging of coronary arteries: comparison of intra- and extravascular contrast agents in swine Radiology. 2001;218:670-8.

[48] Miyazaki M., Lee V. S.. Nonenhanced MR angiography Radiology. 2008;248:20-43.

[49] Prince M. R.. Gadolinium-enhanced MR aortography Radiology. 1994;191:155-64.

[50] Prince M. R., Chabra S. G., Watts R., et al. Contrast material travel times in patients undergoing peripheral MR angiography Radiology. 2002;224:55-61.

[51] Lee J. J., Chang Y., Tirman P. J., et al. Optimizing of gadolinium-enhanced MR angiography by manipulation of acquisition and scan delay times Eur Radiol. 2001;11:754-66.

[52] Foo T. K., Saranathan M., Prince M. R., Chenevert T. L.. Automated detection of bolus arrival and initiation of data acquisition in fast, three-dimensional, gadoliniumenhanced MR angiography Radiology. 1997;203:275-80.

[53] Prince M. R., Chenevert T. L., Foo T. K., Londy F. J., Ward J. S., Maki J. H.. Contrast-enhanced abdominal MR angiography: optimization of imaging delay time by automating the detection of contrast material arrival in the aorta Radiology. 1997;203:109-14.

[54] Ho V. B., Foo T. K.. Optimization of gadolinium-enhanced magnetic resonance angiography using an automated bolus-detection algorithm (MR SmartPrep). Original investigation Invest Radiol. 1998;33:515-23.

[55] Riederer S. J., Bernstein M. A., Breen J. F., et al. Three-dimensional contrastenhanced MR angiography with real-time fluoroscopic triggering: design specifications and technical reliability in 330 patient studies Radiology. 2000;215:584-93.

[56] Wang Y., Winchester P. A., Khilnani N. M., et al. Contrast-enhanced peripheral MR angiography from the abdominal aorta to the pedal arteries: combined dynamic two-dimensional and bolus-chase three-dimensional acquisitions Invest Radiol. 2001;36:170-7.

[57] Bonel H. M., Saar B., Hoppe H., et al. MR angiography of infrapopliteal arteries in patients with peripheral arterial occlusive disease by using Gadofosveset at 3.0 T: diagnostic accuracy compared with selective DSA Radiology. 2009;253:879-90.

[58] Jaspers K., Nijenhuis R. J., Backes W. H.. Differentiation of spinal cord arteries and veins by time-resolved MR angiography J Magn Reson Imaging. 2007;26:31-40.

[59] Bremerich J., Bilecen D., Reimer P.. MR angiography with blood pool contrast agents Eur Radiol. 2007;17:3017-24.

[60] Longland C. J.. Collateral circulation in the limb Postgrad Med J. 1953;29:456-8.

[61] Fuchs S., Shou M., Baffour R., Epstein S. E., Kornowski R.. Lack of correlation between angiographic grading of collateral and myocardial perfusion and function: implications for the assessment of angiogenic response Coron Artery Dis. 2001;12:1738. 
[62] Bondke A., Buschmann I. R.. Visualization of therapeutic arteriogenesis by MR angiography: functional measurements are superior to surrogate parameters $J$ Magn Reson Imaging. 2007;26:215; author reply 216.

[63] Lebon V., Carlier P. G., Brillault-Salvat C., Leroy-Willig A.. Simultaneous measurement of perfusion and oxygenation changes using a multiple gradient-echo sequence: application to human muscle study Magn Reson Imaging. 1998;16:721-9.

[64] Ledermann H. P., Schulte A. C., Heidecker H. G., et al. Blood oxygenation leveldependent magnetic resonance imaging of the skeletal muscle in patients with peripheral arterial occlusive disease Circulation. 2006;113:2929-35.

[65] Noseworthy M. D., Bulte D. P., Alfonsi J.. BOLD magnetic resonance imaging of skeletal muscle Semin Musculoskelet Radiol. 2003;7:307-15.

[66] Wu B., Wang X., Guo J., et al. Collateral circulation imaging: MR perfusion territory arterial spin-labeling at 3T AJNR Am J Neuroradiol. 2008;29:1855-60.

[67] Wu W. C., Mohler 3rd, Ratcliffe S. J., Wehrli F. W., Detre J. A., Floyd T. F.. Skeletal muscle microvascular flow in progressive peripheral artery disease: assessment with continuous arterial spin-labeling perfusion magnetic resonance imaging $\mathrm{J} \mathrm{Am}$ Coll Cardiol. 2009;53:2372-7.

[68] Padhani A. R.. Dynamic contrast-enhanced MRI in clinical oncology: current status and future directions J Magn Reson Imaging. 2002;16:407-22.

[69] Barrett T., Kobayashi H., Brechbiel M., Choyke P. L.. Macromolecular MRI contrast agents for imaging tumor angiogenesis Eur J Radiol. 2006;60:353-66.

[70] Brix G., Kiessling F., Lucht R., et al. Microcirculation and microvasculature in breast tumors: pharmacokinetic analysis of dynamic MR image series Magn Reson Med. 2004;52:420-9.

[71] Lussanet Q. G., Backes W. H., Griffioen A. W., et al. Dynamic contrast-enhanced magnetic resonance imaging of radiation therapy-induced microcirculation changes in rectal cancer Int J Radiat Oncol Biol Phys. 2005;63:1309-15.

[72] Lussanet Q. G., Beets-Tan R. G., Backes W. H., et al. Dynamic contrast-enhanced magnetic resonance imaging at 1.5 Tesla with gadopentetate dimeglumine to assess the angiostatic effects of anginex in mice Eur J Cancer. 2004;40:1262-8.

[73] Su M. Y., Wang Z., Nalcioglu O.. Investigation of longitudinal vascular changes in control and chemotherapy-treated tumors to serve as therapeutic efficacy predictors J Magn Reson Imaging. 1999;9:128-37.

[74] Turetschek K., Preda A., Novikov V., et al. Tumor microvascular changes in antiangiogenic treatment: assessment by magnetic resonance contrast media of different molecular weights J Magn Reson Imaging. 2004;20:138-44.

[75] Ziv K., Nevo N., Dafni H., et al. Longitudinal MRI tracking of the angiogenic response to hind limb ischemic injury in the mouse Magn Reson Med. 2004;51:304-11.

[76] Lussanet Q. G., Golde J. C., Beets-Tan R. G., et al. Dynamic contrast-enhanced MRI of muscle perfusion combined with MR angiography of collateral artery growth in a femoral artery ligation model NMR Biomed. 2007;20:717-25. 
[77] Faranesh A. Z., Kraitchman D. L., McVeigh E. R.. Measurement of kinetic parameters in skeletal muscle by magnetic resonance imaging with an intravascular agent Magn Reson Med. 2006;55:1114-23.

[78] Thompson R. B., Aviles R. J., Faranesh A. Z., et al. Measurement of skeletal muscle perfusion during postischemic reactive hyperemia using contrast-enhanced MRI with a step-input function Magn Reson Med. 2005;54:289-98.

[79] Walker-Samuel S., Leach M. O., Collins D. J.. Evaluation of response to treatment using DCE-MRI: the relationship between initial area under the gadolinium curve (IAUGC) and quantitative pharmacokinetic analysis Phys Med Biol. 2006;51:3593-602.

[80] Galbraith S. M., Lodge M. A., Taylor N. J., et al. Reproducibility of dynamic contrastenhanced MRI in human muscle and tumours: comparison of quantitative and semiquantitative analysis NMR Biomed. 2002;15:132-42.

[81] St Lawrence K. S., Lee T. Y.. An adiabatic approximation to the tissue homogeneity model for water exchange in the brain: I. Theoretical derivation J Cereb Blood Flow Metab. 1998;18:1365-77.

[82] Tofts P. S.. Modeling tracer kinetics in dynamic Gd-DTPA MR imaging J Magn Reson Imaging. 1997;7:91-101.

[83] Tofts P. S., Brix G., Buckley D. L., et al. Estimating kinetic parameters from dynamic contrast-enhanced T(1)-weighted MRI of a diffusable tracer: standardized quantities and symbols J Magn Reson Imaging. 1999;10:223-32.

[84] Patlak C. S., Blasberg R. G., Fenstermacher J. D.. Graphical evaluation of blood-tobrain transfer constants from multiple-time uptake data J Cereb Blood Flow Metab. 1983;3:1-7.

[85] Goldstein T. A., Jerosch-Herold M., Misselwitz B., Zhang H., Gropler R. J., Zheng J.. Fast mapping of myocardial blood flow with MR first-pass perfusion imaging Magn Reson Med. 2008;59:1394-400.

[86] Luo Y., Mohning K. M., Hradil V. P., et al. Evaluation of tissue perfusion in a rat model of hind-limb muscle ischemia using dynamic contrast-enhanced magnetic resonance imaging J Magn Reson Imaging. 2002;16:277-83.

[87] Aerts H. J., Riel N. A., Backes W. H.. System identification theory in pharmacokinetic modeling of dynamic contrast-enhanced MRI: Influence of contrast injection Magn Reson Med. 2008;59:1111-9.

[88] Henderson E., Rutt B. K., Lee T. Y.. Temporal sampling requirements for the tracer kinetics modeling of breast disease Magn Reson Imaging. 1998;16:1057-73.

[89] Lopata R. G., Backes W. H., Bosch P. P., Riel N. A.. On the identifiability of pharmacokinetic parameters in dynamic contrast-enhanced imaging Magn Reson Med. 2007;58:425-9.

[90] Lussanet Q. G., Langereis S., Beets-Tan R. G., et al. Dynamic contrast-enhanced MR imaging kinetic parameters and molecular weight of dendritic contrast agents in tumor angiogenesis in mice Radiology. 2005;235:65-72. 
[91] Adam G., Muhler A., Spuntrup E., et al. Differentiation of spontaneous canine breast tumors using dynamic magnetic resonance imaging with 24-Gadolinium-DTPAcascade-polymer, a new blood-pool agent. Preliminary experience Invest Radiol. 1996;31:267-74.

[92] Daldrup-Link H. E., Shames D. M., Wendland M., et al. Comparison of Gadomer-17 and gadopentetate dimeglumine for differentiation of benign from malignant breast tumors with MR imaging Acad Radiol. 2000;7:934-44.

[93] Su M. Y., Wang Z., Carpenter P. M., Lao X., Muhler A., Nalcioglu O.. Characterization of $\mathrm{N}$-ethyl- $\mathrm{N}$-nitrosourea-induced malignant and benign breast tumors in rats by using three MR contrast agents J Magn Reson Imaging. 1999;9:177-86.

[94] Schirmer S. H., Nooijen F. C., Piek J. J., Royen N.. Stimulation of collateral artery growth: travelling further down the road to clinical application Heart. 2009;95:191-7.

[95] Backes W. H., Nijenhuis R. J., Mess W. H., Wilmink F. A., Schurink G. W., Jacobs M. J.. Magnetic resonance angiography of collateral blood supply to spinal cord in thoracic and thoracoabdominal aortic aneurysm patients J Vasc Surg. 2008;48:26171.

[96] Baumgartner I., Thoeny H. C., Kummer O., et al. Leg ischemia: assessment with MR angiography and spectroscopy Radiology. 2005;234:833-41.

[97] Lussanet Q. G., Golde J. C., Beets-Tan R. G., et al. Magnetic resonance angiography of collateral vessel growth in a rabbit femoral artery ligation model NMR Biomed. 2006; 19:77-83.

[98] Wecksell M. B., Winchester P. A., Bush Jr., Kent K. C., Prince M. R., Wang Y.. Cross-sectional pattern of collateral vessels in patients with superficial femoral artery occlusion Invest Radiol. 2001;36:422-9.

[99] Swan J. S., Carroll T. J., Kennell T. W., et al. Time-resolved three-dimensional contrast-enhanced MR angiography of the peripheral vessels Radiology. 2002;225:4352.

[100] Rohrer M., Bauer H., Mintorovitch J., Requardt M., Weinmann H. J.. Comparison of magnetic properties of MRI contrast media solutions at different magnetic field strengths Invest Radiol. 2005;40:715-24.

[101] Dong Q., Hurst D. R., Weinmann H. J., Chenevert T. L., Londy F. J., Prince M. R.. Magnetic resonance angiography with gadomer-17. An animal study original investigation Invest Radiol. 1998;33:699-708.

[102] Clarke S. E., Weinmann H. J., Dai E., Lucas A. R., Rutt B. K.. Comparison of two blood pool contrast agents for 0.5-T MR angiography: experimental study in rabbits Radiology. 2000;214:787-94.

[103] Maki J. H., Wang M., Wilson G. J., Shutske M. G., Leiner T.. Highly accelerated first-pass contrast-enhanced magnetic resonance angiography of the peripheral vasculature: comparison of gadofosveset trisodium with gadopentetate dimeglumine contrast agents J Magn Reson Imaging. 2009;30:1085-92. 
References

[104] Corot C., Violas X., Robert P., Gagneur G., Port M.. Comparison of different types of blood pool agents (P792, MS325, USPIO) in a rabbit MR angiography-like protocol Invest Radiol. 2003;38:311-9.

[105] Leiner T., Goyen M., Rohrer M., Schoenberg S. O.. Clinical Blood Pool MR Imaging. Heidelberg: Springer Medizin Verlag 2008.

[106] Port M., Corot C., Raynal I., et al. Physicochemical and biological evaluation of P792, a rapid-clearance blood-pool agent for magnetic resonance imaging Invest Radiol. 2001;36:445-54.

[107] Port M., Corot C., Rousseaux O., et al. P792: a rapid clearance blood pool agent for magnetic resonance imaging: preliminary results Magma. 2001;12:121-7.

[108] Misselwitz B., Schmitt-Willich H., Ebert W., Frenzel T., Weinmann H. J.. Pharmacokinetics of Gadomer-17, a new dendritic magnetic resonance contrast agent Magma. 2001;12:128-34.

[109] Herborn C. U., Schmidt M., Bruder O., Nagel E., Shamsi K., Barkhausen J.. MR coronary angiography with SH L 643 A: initial experience in patients with coronary artery disease Radiology. 2004;233:567-73.

[110] Nielsen Y. W., Eiberg J. P., Logager V. B., Hansen M. A., Schroeder T. V., Thomsen H. S.. Whole-body MR angiography with body coil acquisition at $3 \mathrm{~T}$ in patients with peripheral arterial disease using the contrast agent gadofosveset trisodium Acad Radiol. 2009;16:654-61.

[111] Hadizadeh D. R., Gieseke J., Lohmaier S. H., et al. Peripheral MR angiography with blood pool contrast agent: prospective intraindividual comparative study of highspatial-resolution steady-state MR angiography versus standard-resolution first-pass MR angiography and DSA Radiology. 2008;249:701-11.

[112] Jaspers K., Aerts H. J., Leiner T., et al. Reliability of pharmacokinetic parameters: small vs. medium-sized contrast agents Magn Reson Med. 2009;62:779-87.

[113] Zhuang Z. W., Gao L., Murakami M., et al. Arteriogenesis: noninvasive quantification with multi-detector row $C T$ angiography and three-dimensional volume rendering in rodents Radiology. 2006;240:698-707.

[114] Jaspers K., Leiner T., Dijkstra P., et al. Optimized pharmacokinetic modeling for the detection of perfusion differences in skeletal muscle with DCE-MRI: Effect of contrast agent size Med Phys. 2010;37:5746-5755.

[115] Stanisz G. J., Odrobina E. E., Pun J., et al. T1, T2 relaxation and magnetization transfer in tissue at 3T Magn Reson Med. 2005;54:507-12.

[116] Hendrick R., Roff U.. Image contrast and noise in Magnetic Resonance Imaging (Stark D., Bradley W.. , eds.):135Chicago: Moshby Yearbook 1991.

[117] Attanasio S., Snell J.. Therapeutic angiogenesis in the management of critical limb ischemia: current concepts and review Cardiol Rev. 2009;17:115-20.

[118] Grundmann S., Piek J. J., Pasterkamp G., Hoefer I. E.. Arteriogenesis: basic mechanisms and therapeutic stimulation Eur J Clin Invest. 2007;37:755-66. 
[119] Wagner S., Helisch A., Bachmann G., Schaper W.. Time-of-flight quantitative measurements of blood flow in mouse hindlimbs J Magn Reson Imaging. 2004;19:468-74.

[120] Wagner S., Helisch A., Ziegelhoeffer T., Bachmann G., Schaper W.. Magnetic resonance angiography of collateral vessels in a murine femoral artery ligation model NMR Biomed. 2004;17:21-7.

[121] Greve J. M., Chico T. J., Goldman H., et al. Magnetic resonance angiography reveals therapeutic enlargement of collateral vessels induced by VEGF in a murine model of peripheral arterial disease J Magn Reson Imaging. 2006;24:1124-32.

[122] Jacoby C., Boring Y. C., Beck A., et al. Dynamic changes in murine vessel geometry assessed by high-resolution magnetic resonance angiography: a 9.4T study $J$ Magn Reson Imaging. 2008;28:637-45.

[123] Chapman B. E., Stapelton J. O., Parker D. L.. Intracranial vessel segmentation from time-of-flight MRA using pre-processing of the MIP Z-buffer: accuracy of the ZBS algorithm Med Image Anal. 2004;8:113-26.

[124] Sun Y., Parker D. L.. Performance analysis of maximum intensity projection algorithm for display of MRA images IEEE Trans Med Imaging. 1999;18:1154-69.

[125] Bennink H. E., Assen H. C., Streekstra G. J., Wee R., Spaan J. A., Haar Romeny B. M.. A novel 3D multi-scale lineness filter for vessel detection Med Image Comput Comput Assist Interv. 2007;10:436-43.

[126] Kagadis G. C., Spyridonos P., Karnabatidis D., et al. Computerized analysis of digital subtraction angiography: a tool for quantitative in-vivo vascular imaging J Digit Imaging. 2008;21:433-45.

[127] Frangi A.F., Niessen W.J., Vincken K.L., Viergever M.A.. Multiscale vessel enhancement filtering Proceeding of the International Conference on Medical Image Computing Computer-assisted Intervention, Lect. Notes Comp. Sci, 1496. 1998;Lect. Notes Comp. Sci, 1496:130-137.

[128] Lesage D., Angelini E. D., Bloch I., Funka-Lea G.. A review of 3D vessel lumen segmentation techniques: models, features and extraction schemes Med Image Anal. 2009;13:819-45.

[129] Wilson D. L., Noble J. A.. An adaptive segmentation algorithm for time-of-flight MRA data IEEE Trans Med Imaging. 1999;18:938-45.

[130] Hassouna M. S., Farag A. A., Hushek S., Moriarty T.. Cerebrovascular segmentation from TOF using stochastic models Med Image Anal. 2006;10:2-18.

[131] Boetes C., Barentsz J. O., Mus R. D., et al. MR characterization of suspicious breast lesions with a gadolinium-enhanced TurboFLASH subtraction technique Radiology. 1994;193:777-81.

[132] Pathak A. P., Artemov D., Ward B. D., Jackson D. G., Neeman M., Bhujwalla Z. M.. Characterizing extravascular fluid transport of macromolecules in the tumor interstitium by magnetic resonance imaging Cancer Res. 2005;65:1425-32.

[133] Pellerin M., Yankeelov T. E., Lepage M.. Incorporating contrast agent diffusion into the analysis of DCE-MRI data Magn Reson Med. 2007;58:1124-34. 
[134] Koh T. S., Cheong L. H., Hou Z., Soh Y. C.. A physiologic model of capillary-tissue exchange for dynamic contrast-enhanced imaging of tumor microcirculation IEEE Trans Biomed Eng. 2003;50:159-67.

[135] Murase K.. Efficient method for calculating kinetic parameters using T1weighted dynamic contrast-enhanced magnetic resonance imaging Magn Reson Med. 2004;51:858-62.

[136] Yankeelov T. E., Luci J. J., Lepage M., et al. Quantitative pharmacokinetic analysis of DCE-MRI data without an arterial input function: a reference region model Magn Reson Imaging. 2005;23:519-29.

[137] Bourasset F., Dencausse A., Bourrinet P., Ducret M., Corot C.. Comparison of plasma and peritoneal concentrations of various categories of MRI blood pool agents in a murine experimental pharmacokinetic model Magma. 2001;12:82-7.

[138] Preda A., Vliet M., Krestin G. P., Brasch R. C., Dijke C. F.. Magnetic resonance macromolecular agents for monitoring tumor microvessels and angiogenesis inhibition Invest Radiol. 2006;41:325-31.

[139] Roberts H. C., Saeed M., Roberts T. P., et al. Comparison of albumin-(Gd-DTPA)30 and Gd-DTPA-24-cascade-polymer for measurements of normal and abnormal microvascular permeability J Magn Reson Imaging. 1997;7:331-8.

[140] Buckley D. L.. Uncertainty in the analysis of tracer kinetics using dynamic contrastenhanced T1-weighted MRI Magn Reson Med. 2002;47:601-6.

[141] Lopata R. G., Riel N. A.. Identifiability analysis of the standard pharmacokinetic models in DCE MR imaging of tumours Conf Proc IEEE Eng Med Biol Soc. 2004;2:1040-3.

[142] Faranesh A. Z., Yankeelov T. E.. Incorporating a vascular term into a reference region model for the analysis of DCE-MRI data: a simulation study Phys Med Biol. 2008;53:2617-31.

[143] Padhani A. R., Hayes C., Landau S., Leach M. O.. Reproducibility of quantitative dynamic MRI of normal human tissues NMR Biomed. 2002;15:143-53.

[144] Daldrup H. E., Shames D. M., Husseini W., Wendland M. F., Okuhata Y., Brasch R. C.. Quantification of the extraction fraction for gadopentetate across breast cancer capillaries Magn Reson Med. 1998;40:537-43.

[145] Jones W. S., Annex B. H.. Growth factors for therapeutic angiogenesis in peripheral arterial disease Curr Opin Cardiol. 2007;22:458-63.

[146] Zahra M. A., Hollingsworth K. G., Sala E., Lomas D. J., Tan L. T.. Dynamic contrastenhanced MRI as a predictor of tumour response to radiotherapy Lancet Oncol. 2007;8:63-74.

[147] Wilke N., Jerosch-Herold M., Wang Y., et al. Myocardial perfusion reserve: assessment with multisection, quantitative, first-pass MR imaging Radiology. 1997;204:373-84.

[148] Al-Saadi N., Nagel E., Gross M., et al. Noninvasive detection of myocardial ischemia from perfusion reserve based on cardiovascular magnetic resonance Circulation. 2000;101:1379-83. 
[149] Cheng H. L.. T1 measurement of flowing blood and arterial input function determination for quantitative 3D T1-weighted DCE-MRI J Magn Reson Imaging. 2007;25:1073-8.

[150] Donaldson S. B., West C. M., Davidson S. E., et al. A comparison of tracer kinetic models for T1-weighted dynamic contrast-enhanced MRI: application in carcinoma of the cervix Magn Reson Med. 2010;63:691-700.

[151] Hoefer I. E., Royen N., Buschmann I. R., Piek J. J., Schaper W.. Time course of arteriogenesis following femoral artery occlusion in the rabbit Cardiovasc Res. 2001;49:60917.

[152] Schaper W., Schaper J.. Arteriogenesis. Boston, Dordrecht, London: Kluwer Academic Publishers 2004.

[153] Radegran G., Calbet J. A.. Role of adenosine in exercise-induced human skeletal muscle vasodilatation Acta Physiol Scand. 2001;171:177-85.

[154] Fram E. K., Herfkens R. J., Johnson G. A., et al. Rapid calculation of T1 using variable flip angle gradient refocused imaging Magn Reson Imaging. 1987;5:201-8.

[155] Haacke E.M., Brown R.W, Thompson M.R., Venkatesan R.. Magnetic resonance imaging: physical principles and sequence design. New York: John Wiley \& Sons, Inc. 1999.

[156] Ewing J. R., Brown S. L., Lu M., et al. Model selection in magnetic resonance imaging measurements of vascular permeability: Gadomer in a $9 \mathrm{~L}$ model of rat cerebral tumor J Cereb Blood Flow Metab. 2006;26:310-20.

[157] Bates D., Watts D.. Nonlinear regression analysis and its applications. New York: John Wiley \& Sons, Inc. 1988.

[158] Evelhoch J. L.. Key factors in the acquisition of contrast kinetic data for oncology $J$ Magn Reson Imaging. 1999;10:254-9.

[159] Gerlowski L. E., Jain R. K.. Microvascular permeability of normal and neoplastic tissues Microvasc Res. 1986;31:288-305.

[160] Ludemann L., Schmitt B., Podrabsky P., Schnackenburg B., Bock J., Gutberlet M.. Usage of the $\mathrm{T} 1$ effect of an iron oxide contrast agent in an animal model to quantify myocardial blood flow by MRI Eur J Radiol. 2007;62:247-56.

[161] Turetschek K., Floyd E., Shames D. M., et al. Assessment of a rapid clearance blood pool MR contrast medium (P792) for assays of microvascular characteristics in experimental breast tumors with correlations to histopathology Magn Reson Med. 2001;45:880-6.

[162] Jacquier A., Wendland M., Do L., et al. MR imaging assessment of the kinetics of P846, a new gadolinium-based MR contrast medium, in ischemically injured myocardium Contrast Media Mol Imaging. 2008;3:112-9.

[163] Lauffer R. B., Parmelee D. J., Dunham S. U., et al. MS-325: albumin-targeted contrast agent for MR angiography Radiology. 1998;207:529-38. 
[164] Turetschek K., Floyd E., Helbich T., et al. MRI assessment of microvascular characteristics in experimental breast tumors using a new blood pool contrast agent (MS-325) with correlations to histopathology J Magn Reson Imaging. 2001;14:237-42.

[165] Gerber B. L., Bluemke D. A., Chin B. B., et al. Single-vessel coronary artery stenosis: myocardial perfusion imaging with Gadomer-17 first-pass MR imaging in a swine model of comparison with gadopentetate dimeglumine Radiology. 2002;225:104-12.

[166] Choi K. M., Kim R. J., Gubernikoff G., Vargas J. D., Parker M., Judd R. M.. Transmural extent of acute myocardial infarction predicts long-term improvement in contractile function Circulation. 2001;104:1101-7.

[167] Dormandy J., Heeck L., Vig S.. The fate of patients with critical leg ischemia Semin Vasc Surg. 1999;12:142-7.

[168] Favier J., Germain S., Emmerich J., Corvol P., Gasc J.M.. Critical overexpression of thrombospondin 1 in chronic leg ischaemia J Pathol. 2005;207:358-366.

[169] Schierling W., Troidl K., Troidl C., Schmitz-Rixen T., Schaper W., Eitenmuller I. K.. The role of angiogenic growth factors in arteriogenesis J Vasc Res. 2009;46:365-74.

[170] Ahn A., Frishman W. H., Gutwein A., Passeri J., Nelson M.. Therapeutic angiogenesis: a new treatment approach for ischemic heart disease-part I Cardiol Rev. 2008;16:16371.

[171] Rissanen T. T., Markkanen J. E., Arve K., et al. Fibroblast growth factor 4 induces vascular permeability, angiogenesis and arteriogenesis in a rabbit hindlimb ischemia model Faseb J. 2003;17:100-2.

[172] Vajanto I., Rissanen T. T., Rutanen J., et al. Evaluation of angiogenesis and side effects in ischemic rabbit hindlimbs after intramuscular injection of adenoviral vectors encoding VEGF and LacZ J Gene Med. 2002;4:371-80.

[173] Higgins C. B., Botvinick E. H., Lanzer P., et al. Cardiovascular imaging with nuclear magnetic resonance Cardiol Clin. 1983;1:527-39.

[174] Edwards N. C., Routledge H., Steeds R. P.. T2-weighted magnetic resonance imaging to assess myocardial oedema in ischaemic heart disease Heart. 2009;95:1357-61.

[175] Kattapuram T. M., Suri R., Rosol M. S., Rosenberg A. E., Kattapuram S. V.. Idiopathic and diabetic skeletal muscle necrosis: evaluation by magnetic resonance imaging Skeletal Radiol. 2005;34:203-9.

[176] Helisch A., Wagner S., Khan N., et al. Impact of mouse strain differences in innate hindlimb collateral vasculature Arterioscler Thromb Vasc Biol. 2006;26:520-6.

[177] Masaki I., Yonemitsu Y., Yamashita A., et al. Angiogenic gene therapy for experimental critical limb ischemia: acceleration of limb loss by overexpression of vascular endothelial growth factor 165 but not of fibroblast growth factor-2 Circ Res. 2002;90:966-73.

[178] Hogers B., Gross D., Lehmann V., et al. Magnetic resonance microscopy at 17.6-Tesla on chicken embryos in vitro J Magn Reson Imaging. 2001;14:83-6. 
[179] Anzidei M., Napoli A., Marincola B. C., et al. Gadofosveset-enhanced MR angiography of carotid arteries: does steady-state imaging improve accuracy of first-pass imaging? Comparison with selective digital subtraction angiography Radiology. 2009;251:45766.

[180] Versluis B., Backes W. H., Eupen M. G., et al. Magnetic resonance imaging in peripheral arterial disease: reproducibility of the assessment of morphological and functional vascular status Invest Radiol. 2010;46:11-24.

[181] Friedrich M. G., Niendorf T., Schulz-Menger J., Gross C. M., Dietz R.. Blood oxygen level-dependent magnetic resonance imaging in patients with stress-induced angina Circulation. 2003;108:2219-23.

[182] Egred M., Waiter G. D., Semple S. I., et al. Blood oxygen level-dependent (BOLD) magnetic resonance imaging in patients with dypiridamole induced ischaemia; a PET comparative study Int J Cardiol. 2007;115:36-41.

[183] Ledermann H. P., Heidecker H. G., Schulte A. C., et al. Calf muscles imaged at BOLD MR: correlation with TCPO2 and flowmetry measurements during ischemia and reactive hyperemia-initial experience Radiology. 2006;241:477-84.

[184] Jahnke C., Gebker R., Manka R., Schnackenburg B., Fleck E., Paetsch I.. Navigatorgated 3D blood oxygen level-dependent CMR at 3.0-T for detection of stress-induced myocardial ischemic reactions JACC Cardiovasc Imaging. 2010;3:375-84.

[185] Buxton R. B., Frank L. R., Wong E. C., Siewert B., Warach S., Edelman R. R.. A general kinetic model for quantitative perfusion imaging with arterial spin labeling Magn Reson Med. 1998;40:383-96.

[186] Carlier P. G., Bertoldi D., Baligand C., Wary C., Fromes Y.. Muscle blood flow and oxygenation measured by NMR imaging and spectroscopy NMR Biomed. 2006;19:95467.

[187] Zhang H., Shea S. M., Park V., et al. Accurate myocardial T1 measurements: toward quantification of myocardial blood flow with arterial spin labeling Magn Reson Med. 2005;53:1135-42.

[188] Wang D. J., Bi X., Avants B. B., Meng T., Zuehlsdorff S., Detre J. A.. Estimation of perfusion and arterial transit time in myocardium using free-breathing myocardial arterial spin labeling with navigator-echo Magn Reson Med. 2010;64:1289-95.

[189] Raynaud J. S., Duteil S., Vaughan J. T., et al. Determination of skeletal muscle perfusion using arterial spin labeling NMRI: validation by comparison with venous occlusion plethysmography Magn Reson Med. 2001;46:305-11.

[190] Marro K. I., Hyyti O. M., Vincent M. A., Kushmerick M. J.. Validation and advantages of FAWSETS perfusion measurements in skeletal muscle NMR Biomed. 2005;18:22634.

[191] Ruiter M. S., Golde J. M., Schaper N. C., Stehouwer C. D., Huijberts M. S.. Diabetes impairs arteriogenesis in the peripheral circulation: review of molecular mechanisms Clin Sci (Lond). 2010;119:225-38.

[192] Goyen M., Shamsi K., Schoenberg S. O.. Vasovist-enhanced MR angiography Eur Radiol. 2006;16 Suppl 2:B9-14. 
[193] Jerosch-Herold M., Hu X., Murthy N. S., Rickers C., Stillman A. E.. Magnetic resonance imaging of myocardial contrast enhancement with MS-325 and its relation to myocardial blood flow and the perfusion reserve J Magn Reson Imaging. 2003;18:54454.

[194] Kraitchman D. L., Chin B. B., Heldman A. W., Solaiyappan M., Bluemke D. A.. MRI detection of myocardial perfusion defects due to coronary artery stenosis with MS-325 J Magn Reson Imaging. 2002;15:149-58.

[195] Massoud T. F., Gambhir S. S.. Molecular imaging in living subjects: seeing fundamental biological processes in a new light Genes Dev. 2003;17:545-80.

[196] Kiessling F., Morgenstern B., Zhang C.. Contrast agents and applications to assess tumor angiogenesis in vivo by magnetic resonance imaging Curr Med Chem. 2007;14:7791.

[197] Oostendorp M., Douma K., Wagenaar A., et al. Molecular magnetic resonance imaging of myocardial angiogenesis after acute myocardial infarction Circulation. 2010;121:775-83.

[198] Winter P. M., Caruthers S. D., Lanza G. M., Wickline S. A.. Quantitative cardiovascular magnetic resonance for molecular imaging J Cardiovasc Magn Reson. 2010;12:62. 




\section{Summary}

Peripheral and coronary artery disease (PAD and CAD, respectively) are major causes of death and disability in the industrialized world. These conditions are characterized by stenosis or occlusion of the arteries feeding the extremities and myocardium, respectively. Treatment strategies for PAD and CAD aim at restoring the blood flow to ischemic tissue. Traditionally, this is accomplished by creating a bypass or by placing a stent in the stenosis. However, in a large group of patients, these invasive procedures are not an option or have limited effect. These patients may benefit from therapeutic neovascularization.

Neovascularization, or the development of new blood vessels, plays an important role in many physiological and pathological processes. In adults, two main forms can be distinguished: (i) the sprouting of capillaries (angiogenesis) and (ii) the development of pre-existing arterioles into functional collateral arteries (arteriogenesis). Extensive research has identified several cytokines that play a role in either or both processes. In animal models, administration of such growth factors has been shown to have a positive effect on the development of collateral arteries. However, clinical studies revealed only moderate efficacy with regard to functional end points, so the clinical application needs further improvement. Measuring the early vascular response may enable individual tailoring of treatment, which may result in improved therapeutic efficacy. The development and clinical introduction of therapeutic neovascularization would therefore benefit greatly from the availability of imaging techniques that can reliably detect and monitor early treatment effects. Due to its non-invasive nature, absence of ionizing radiation and versatility, magnetic resonance imaging (MRI) may be considered the most adequate modality for both functional and morphological evaluation of neovascularization.

This thesis discusses several MR techniques that can be used for morphological and functional monitoring of neovascularization therapies. The main focus of this research project was to provide solutions to the specific demands of neovascularization imaging.

Chapter 1 provides an introduction to therapeutic neovascularization. Subsequently, it gives an overview of the MR techniques available for morphological and functional monitoring, and the challenges related to the imaging of neovascularization.

Chapters 2 and 3 focused on the morphological evaluation of the arteriogenic response with MR angiography (MRA). Chapter 2 compared the 
medium-sized contrast agent Gadomer with the clinically available small contrast agent Gd-DTPA with respect to visualization of collateral arteries by steady-state MRA in a rabbit hind limb ischemia model. We demonstrated that Gadomer is suitable for MRA of collateral arteries, as its use perceptibly improved contrast-to-noise characteristics compared to Gd-DTPA, while the dose could be reduced. Interestingly, the realized improvement in visibility of collateral arteries did not result in an increase in the number of identified collaterals, yet it appreciably facilitated the identification of collateral arteries.

In Chapter 3, an automated analysis method for the quantification of collateral artery development was introduced and tested in a rat hind limb ischemia model. Parameters derived from signal intensity distributions were shown to provide information on vascular structures of various sizes. The measures correlated well with the number of collateral arteries found by manual identification. The method is easy to implement, objective, fast and more sensitive to temporal changes than manual identification.

Chapters 4 and 5 explored the use of medium-sized contrast agents in the functional assessment of neovascularization with dynamic contrast-enhanced (DCE) MRI. This technique estimates tissue perfusion based on the extravasation rate of a contrast agent. Chapter 4 addressed the reliability of pharmacokinetic parameter estimation. Monte Carlo simulations were performed to investigate the influence of DCE-MRI measurement settings on parameter reliability for both a medium-sized and small contrast agent (MCA and SCA, respectively). Theoretically, MCAs provide improved reliability of pharmacokinetic parameter estimation compared to SCAs due to their lower extravasation rate. MCAs allowed lower sampling frequencies than SCAs, while achieving higher reliability for the determination of the extravastion rate $K^{\text {trans }}$ and fractional plasma volume $v_{p}$.

Subsequently, Chapter 5 discussed the optimization of DCE-MRI data analysis for both an MCA and SCA, and investigated the technique's sensitivity for skeletal muscle perfusion differences in a rabbit hind limb ischemia model. We found that the detection of microvascular differences based on both model-based and model-free parameters was most sensitive when the analysis strategy was tailored to the contrast agent used. The MCA was equally sensitive for microvascular differences as the SCA, with the advantage of improved spatial resolution.

In Chapter 6, the potential of combining functional and morphological MR techniques for the in vivo evaluation of therapeutically induced neovascularization was studied in a rat hind limb ischemia model. DCE-MRI, time-of-flight 
MRA and $T_{2}$-weighted imaging were performed at multiple time points to follow the time course of angiogenesis, arteriogenesis and perfusion recovery. MRI allows comprehensive, non-invasive characterization and monitoring of the arteriogenic and angiogenic responses to neovascularization therapy and their effects on tissue perfusion, by demonstrating time- and treatmentdependent differences between ischemic and control limbs.

Finally, in Chapter 7 the developed methods and obtained results of the various studies were integrated to emphasize the need of comprehensive imaging for the evaluation of neovascularization therapy. The future of therapeutic neovascularization and the supportive role of MRI were discussed. 



\section{Samenvatting}

Perifeer en coronair arterieel vaatlijden (PAV en CAV) zijn belangrijke doodsoorzaken in de geïndustrialiseerde wereld. Deze condities worden gekenmerkt door vernauwing en verstopping van de arteriën die respectievelijk de extremiteiten en het myocardium van bloed voorzien. De behandelingsmethoden van PAV en CAV zijn erop gericht de bloedtoevoer naar het ischemische weefsel te herstellen. Conventionele methodes bereiken dit door een bypass te creëren of een stent te plaatsen. Voor een grote groep patiënten behoren deze invasieve procedures echter niet tot de mogelijkheden, of hebben slechts een beperkt effect. Deze patiënten zouden baat kunnen hebben bij therapeutische neovascularisatie.

Neovascularisatie, de ontwikkeling van nieuwe bloedvaten, speelt een belangrijke rol in vele fysiologische en pathologische processen. In volwassenen kunnen twee vormen worden onderscheiden: (i) het ontspruiten van capillairen (angiogenese), en (ii) de ontwikkeling van aanwezige arteriolen naar functionele collateraalarteriën (arteriogenese). Inmiddels is er uitvoerig onderzoek gedaan naar de cytokines die een rol spelen in één of beide processen. Proefdieronderzoek heeft laten zien dat het toedienen van zulke groeifactoren een positief effect heeft op de ontwikkeling van collateraalarteriën. In klinische studies werden voorzichtig-positieve resultaten in functionele eindpunten bereikt. Voor klinische implementatie dient de therapie echter nog verder verbeterd te worden. Het meten van de vroege vasculaire respons zou individuele aanpassing van de behandeling mogelijk kunnen maken, hetgeen kan resulteren in een effectievere therapie. De ontwikkeling en klinische introductie van therapeutische neovascularisatie zou daarom veel baat hebben bij de beschikbaarheid van beeldvormende technieken warmee vroege behandelingseffecten gedetecteerd en vervolgd kunnen worden. Vanwege het niet-invasieve en veelzijdige karakter van de techniek en de afwezigheid van ioniserende straling kan beeldvorming gebaseerd op magnetische kernspinresonantie (in het Engels: magnetic resonance imaging, MRI) beschouwd worden als de meest geschikte modaliteit voor zowel functionele als morfologische evaluatie van neovascularisatie.

In dit proefschrift worden een aantal morfologische en functionele MRItechnieken besproken die gebruikt kunnen worden voor het monitoren van neovascularisatietherapieën. De belangrijkste focus van dit onderzoeksproject lag bij beiden van oplossingen voor problemen die specifiek zijn voor de beeldvorming met MRI van neovascularisatie. 
Hoofdstuk 1 geeft een introductie in therapeutische neovascularisatie. Vervolgens wordt er een overzicht gegeven van MRI-technieken die geschikt zijn voor het morfologisch en functioneel monitoren van neovascularisatie en de uitdagingen die hiermee samenhangen.

Hoofdstuk 2 en 3 focussen op de morfologische evaluatie van de arteriogene respons met MR-angiografie (MRA). In hoofdstuk 2 wordt het middelgrote contrastmiddel Gadomer vergeleken met het klinisch verkrijgbare, kleine contrastmiddel Gd-DTPA voor het visualiseren van collateraalarteriën met steady-state MRA in een konijnenmodel van perifere ischemie. Wij hebben aangetoond dat Gadomer is geschikt voor MRA van collateraalarteriën, aangezien het gebruik hiervan leidt tot een zichtbare verbetering van de contrast-ruis karakteristieken in vergelijking tot Gd-DTPA, terwijl de dosis gereduceerd kon worden. Opvallend was dat de bereikte verbetering in zichtbaarheid niet resulteerde in een toename van het aantal geïdentificeerde collateralen. Wel was het identificeren van de collateraalarteriën vergemakkelijkt.

In hoofdstuk 3 werd een geautomatiseerde analysemethode om de ontwikkeling van collateraalarteriën te kwantificeren geïntroduceerd en getest in een rattenmodel voor perifere ischemie. Parameters die afgeleid werden uit de verdeling van de signaalintensiteit bleken informatie te verschaffen over vasculaire structuren van verscheidene groottes. Deze parameters vertoonden een goede correlatie met het aantal collateralen dat gevonden werd met handmatige identificatie. De methode is eenvoudig te implementeren, objectief, snel en gevoeliger voor temporele veranderingen dan manuele identificatie.

Hoofdstuk 4 en 5 onderzoeken het gebruik van middelgrote contrastmiddelen in de functionele evaluatie van neovascularisatie met dynamisch contrastversterkte MRI (Engels: dynamic contrast-enhanced MRI, DCE-MRI). DCE-MRI is een techniek waarbij de weefseldoorbloeding geschat wordt op basis van de extravasatiesnelheid van een contrastmiddel. In hoofdstuk 4 wordt de betrouwbaarheid van het schatten van farmacokinetische parameters onderzocht. Met behulp van Monte-Carlo simulaties werd de invloed van MRI-acquisitie-instellingen op deze betrouwbaarheid onderzocht voor zowel een middelgroot als een klein contrastmiddel. Theoretisch gezien bieden middelgrote contrastmiddelen een verhoogde betrouwbaarheid in de schatting van parameters ten opzichte van kleine contrastmiddelen vanwege hun lagere extravasatiesnelheid. Vergeleken met kleine contrastmiddelen konden er voor middelgrote contrastmiddelen lagere scansnelheden worden gekozen, terwijl er een hogere betrouwbaarheid van de bepaling van transferconstante $K^{\text {trans }}$ en fractioneel plasmavolume $v_{\mathrm{p}}$ werd bereikt. 
Vervolgens werd in hoofdstuk 5 de optimalisatie van de data-analyse bij DCE-MRI voor kleine en middelgrote contrastmiddelen onderzocht, en werd de gevoeligheid voor verschillen in de perfusie van skeletspieren bepaald in een konijnenmodel voor perifere ischemie. Het bleek dat de detectie van microvasculaire verschillen gebaseerd op zowel farmcokinetische parameters als modelvrije parameters het gevoeligst was als de analysestrategie werd aangepast aan het gebruikte contrastmiddel. Het middelgrote contrastmiddel bleek even gevoelig voor perfusieverschillen als het kleine contrastmiddel, maar had het voordeel van een betere spatiële resolutie.

In hoofdstuk 6 worden de mogelijkheden van gecombineerde functionele en morfologische MRI-technieken voor de in-vivo-evaluatie van therapeutisch geïnduceerde neovascularisatie bestudeerd in een rattenmodel voor perifere ischemie. DCE-MRI, time-of-flight MR-angiografie en $T_{2}$-gewogen MRI werden uitgevoerd op verschillende tijdstippen om het tijdsverloop van arteriogenese, angiogenese en perfusieherstel te kunnen volgen. MRI maakt uitgebreide noninvasieve karakterisatie en monitoring van de arteriogene en angiogene respons en hun effecten op weefseldoorbloeding mogelijk, doordat er tijds- en therapieafhankelijke verschillen tussen de ischemische en controlepoten aangetoond konden worden.

Ten slotte werden in hoofdstuk 7 de resultaten en bevindingen van de verschillende hoofdstukken samengevat en het belang van beeldvormende technieken die aantonen welke vasculaire veranderingen optreden benadrukt. Ook werden het toekomstperspectief van therapeutische neovascularisatie en de rol van MRI hierbij bediscussieerd. 



\section{Dankwoord}

Het is af! Ruim vier jaar onderzoek is nu samengekomen in dit proefschrift. De weg naar mijn promotie was niet gemakkelijk, maar gelukkig was er een grote groep mensen die mij op vele manieren gesteund en geholpen hebben. Dit proefschrift is dan ook niet compleet zonder een woord van dank voor al deze mensen, van wie ik er een aantal bij naam wil noemen.

Beste Walter, ik wil je bedanken voor je vertrouwen, ook (en vooral) als ik dat zelf niet meer had. Je deur stond altijd open voor kleine en grote problemen. Jouw enthousiasme en betrokkenheid werkten altijd motiverend. Ook wil ik je bedanken dat je mij weer richting de klinische fysica hebt geduwd.

Mark, als mijn promotor was je nauw betrokken bij alle stappen van mijn promotietraject. Bedankt voor je vertrouwen, je goede ideeën, en je inspirerende boekentips. Het was prettig om met je samen te werken.

Tim, met jouw enthousiasme voor wetenschappelijk onderzoek, grote kennis op het gebied van MRA en jouw frisse kijk op mijn onderzoek was je een waardevolle aanvulling op mijn promotieteam. Bedankt!

Beste professor Van Engelshoven, ik heb uw belangstelling voor het verloop van mijn promotieonderzoek en voor mij persoonlijk altijd enorm gewaardeerd. Professor Wildberger, ik wil u bedanken voor de tijd die ik kreeg om dit proefschrift af te ronden.

Beste Jos, je was mijn bondgenoot tijdens de rattenstudies. De vele uren die we samen achter de scanner door hebben gebracht waren nooit saai, dankzij onze gesprekken over ongeveer alles. Met jouw MRI-kennis en geweldige ideeën (kniptang, need I say more?) hebben we die grote klus tot een goed einde gebracht. Bedankt!

Marlies, waar was ik zonder jou geweest? Jouw bijdrage aan de totstandkoming van dit proefschrift bestond niet alleen uit medewerking aan de experimenten en waardevolle adviezen over de manuscripten, maar vooral uit jouw praktische en morele steun als collega, buurvrouw en goede vriendin. Ik ben dan ook heel blij dat jij bij mijn promotie achter me staat als paranimf. 
Er waren 23 konijnen en 58 ratten nodig voor de in dit proefschrift beschreven experimenten, die zonder de hulp van enkele bekwame en gedreven biotechnici niet mogelijk waren geweest. Petra, door jou geweldige inzet en enthousiasme konden de konijnenstudies ook zonder mij doorgaan. Allard, bedankt dat je van deze proefdierneuroot een rattenfan hebt gemaakt. Samen met Niek en Viviane heb jij ervoor gezorgd dat de rattenoperaties voorspoedig verliepen, waarvoor ik jullie alle drie wil bedanken. Mijn dank gaat ook uit naar de medewerkers van de CPV voor de goede verzorging van mijn dieren.

Jolanda, bedankt voor al je hulp bij het opzetten en het uitvoeren van de konijnenstudie. Etiënne en Eveline, met jullie hulp kon het scannen van de konijnen gewoon doorgaan. Bedankt hiervoor!

In het totaal heb ik ruim zes jaar doorgebracht op de onderzoeksgang van de afdeling Radiologie. Het is mede dankzij mijn collega's dat ik mij daar prima thuis voelde. Ik wil jullie daar allemaal hartelijk voor bedanken! Dave en Robbert, bedankt dat ik in mijn pre-promotietijd mee mocht draaien in jullie onderzoeken. Het heeft ervoor gezorgd dat ik ben gebleven. Marc, bedankt voor je hulp bij computerproblemen. Martijn, René en Maarten wil ik bedanken voor de leuke tijd in Stockholm. Eline, René, Martine en Michaela, mijn kamergenootjes van het laatste uur, bedankt voor de gezelligheid en aanmoediging in mijn laatste maanden.

Marcel, Arjen, Hildebrand, Enouschka, Wim en Peter, bedankt voor het warme welkom bij de Klinische Fysica Radiologie in Groningen. Ik heb zin in de komende jaren!

Dit dankwoord is niet compleet zonder een groot dankjewel voor mijn oudstudiegenootjes. Ellen, Karin, Marjon, Cathryn, Anne, Vera, Maud, Ruud, Michiel en Egon, bedankt voor jullie gezelligheid en steun tijdens en na de studie. Ik hoop dat we nog vaak op ME-weekend zullen gaan. Lieve KEMAmeiden, bedankt dat jullie nog een letter wilden toevoegen! Jullie vriendschap is me dierbaar! Lieve Esther, bedankt voor je vriendschap. Hoewel Aarau, Laax, Hasliberg, Leukerbad en Vik goede plaatsen zijn voor lange gesprekken, ben ik erg blij dat we nu gewoon in de bus kunnen stappen om elkaar te zien.

Waar zou ik zijn zonder een fantastische thuisbasis? Lieve familie, Druwerbrinkburen en vrienden uit de gemeenschap Emmen, bedankt dat jullie er voor mij waren en zijn! Ook wil ik mijn 'wintersportfamilie' bedanken: oom Ben, tante Marijke, Linda, Jasper, Dionne, Jan-Willem, Peter en Hanneke. Of er nou geskied wordt of niet, een weekje in de sneeuw samen met jullie geeft energie voor een heel jaar! 
Lieve Sjoerd, ik vind het een eer dat jij mijn paranimf wilt zijn. Ik ben ontzettend trots op je!

Lieve pap en mam, de laatste woorden van dit dankwoord zijn voor jullie. Eigenlijk is het heel simpel: zonder jullie steun had dit proefschrift hier niet gelegen. Ik zou een hoofdstuk kunnen schrijven over wat jullie de afgelopen jaren voor mij betekend hebben, maar ook dat zou nog geen recht doen aan mijn dankbaarheid. Ik stop daarom alles wat ik wil zeggen in een woord: bedankt! 



\section{Scientific publications}

This thesis is based on the following publications:

- Jaspers K, Versluis B, Leiner T, Dijkstra P, Oostendorp M, van Golde JM, Post MJ, Backes WH. MR angiography of collateral arteries: comparison between medium-sized and small contrast agents. PLOS ONE. 2011; 6: e16159.

- Jaspers K, Slenter JM, Leiner T, Wagenaar A, Post MJ, Backes WH. Vessel-enhanced signal intensity distribution analysis for MR angiography of peripheral arteriogenesis. Journal of Magnetic Resonance Imaging. Provisionally accepted.

- Jaspers K, HJWL, Leiner T, Oostendorp M, van Riel NAW, Post MJ and Backes WH. Reliability of pharmacokinetic parameters: small versus medium-sized contrast agents. Magnetic Resonance in Medicine. 2009; 62:779-87.

- Jaspers K, Leiner T, Dijkstra P, Oostendorp M, van Golde JM, Post MJ, Backes WH. Optimized DCE-MRI for detecting perfusion differences in skeletal muscle: effect of contrast agent size and pharmacokinetic model. Medical Physics. 2010; 37:5746-55.

- Jaspers K, Post MJ, Slenter JM, Leiner T, Wagenaar A, Backes WH. MRI of therapeutic neovascularization]Morphological and functional monitoring of therapeutically induced neovascularization in ischemia with MRI. Submitted. 
Other publications:

- Versluis B, Backes WH, van Eupen MG, Jaspers K, Nelemans PJ, Rouwet EV, Teijink JA, Mali WP, Schurink GW, Wildberger JE, Leiner $T$. Magnetic resonance imaging in peripheral arterial disease: reproducibility of the assessment of morphological and functional vascular status. Investigative Radiology. 2011; 46:11-24.

- Jaspers K, Nijenhuis RJ, Backes WH. Differentiation of spinal cord arteries and veins by time-resolved MR angiography. Journal of Magnetic Resonance Imaging. 2007; 26:31-40.

- Nijenhuis RJ, Jacobs MJ, Jaspers K, Reinders M, van Engelshoven $J M$, Leiner $T$, Backes WH. Comparison of magnetic resonance with computed tomography angiography for preoperative localization of the Adamkiewicz artery in thoracoabdominal aortic aneurysm patients. Journal of Vascular Surgery. 2007; 45: 677-85. 


\section{Curriculum vitae}

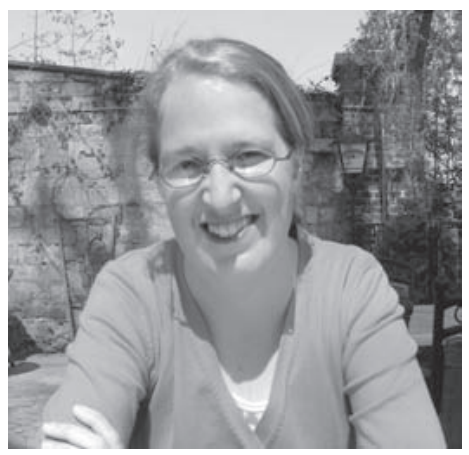

Karolien Jaspers werd geboren op 27 januari 1981 te Hengelo (O). In 1999 behaalde zij haar gymnasiumdiploma aan het Esdalcollege te Emmen. In hetzelfde jaar begon zij met de studie Biomedische Technologie aan de Technische Universiteit Eindhoven. In 2004 startte zij met de masteropleiding Medical Engineering aan dezelfde universiteit. Zij liep stages bij de afdeling Radiologie in het Maastricht Universitair Medisch Centrum (MUMC+) en bij de afdeling Radiotherapie van het Catharina Ziekenhuis te Eindhoven. In 2005 begon zij met haar afstudeerproject bij de afdeling Radiologie van het MUMC+, waar zij onderzoek deed naar differentiële MRA van ruggenmergsarteriën en -venen. Voor dit onderzoek won zij de derde prijs op het Maastricht Medical Students Research Conference 2006. In januari 2006 studeerde zij cum laude af. Van april 2006 tot december 2010 werkte zij aan haar promotieonderzoek met als onderwerp MRI van therapeutische neovascularisatie. De resultaten van dit onderzoek zijn samengebracht in dit proefschrift.

Momenteel is Karolien werkzaam als klinisch fysicus in opleiding bij de afdeling Radiologie van het Universitair Medisch Centrum Groningen. 